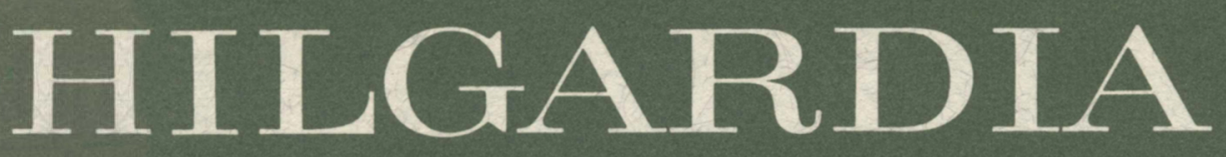

A IOURNAL OF AGRICULTURAL SCIENCE PUBLISHED BY THE CALIFORNIA AGRICULTURAL EXPERIMENT STATION

Volume 42, Number $8 \cdot$ December, 1973

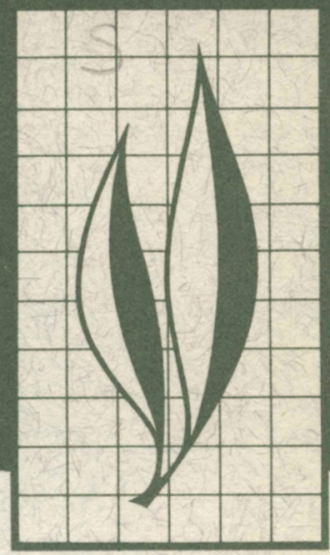

\title{
Report of Diagnoses of Diseased Insects
}

\section{2-1972}

Gerard M. Thomas and

George O. Poinar, Jr.

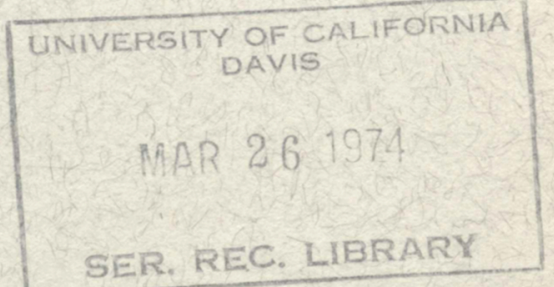

HILGA4 42 (8) 261-360 (1973) 


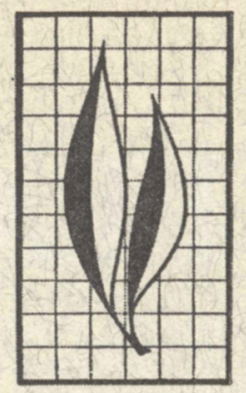

During the 10-year period January 1, 1962-December 31, 1971, our diagnostic laboratory received a total of 1,208 accessions for disease diagnosis. Of these, 1,194 were in the phylum Arthropoda with most $(1,173)$ in the class Insecta. Other arthropod classes represented were Arachnida (16), Crustacea (3), and Symphyla (2). Ten accessions were from the phylum Annelida, one from the phylum Chordata, and three were microbial cultures submitted for identification.

The following report is an abstract listing of the accessions received during this 10 -year period, including brief statements as to the source of the material and the identity of the etiology involved. Each accession is cited under the submitted species. Orders, families, genera and species are in alphabetical order under their respective phyla. Indices to the submitted insects and other hosts (page 351) as well as to the pathogens (page 355), will assist in locating specific organisms.

The microbial pathogens isolated from the 1,208 accessions summarized in this report, including repeats and multiple diagnoses from individual accessions, totaled 88 viruses (52 nuclear polyhedrosis viruses, 7 cytoplasmic polyhedrosis viruses, 24 granulosis viruses, 5 noninclusion viruses), 1 rickettsia, 224 bacteria, 1 algae, 285 fungi, 122 protozoans (100 microsporidians, 18 schizogregarines, 4 coccidians), 5 nematodes, and 1 nematomorph. Entomophagous parasites and predators were found in 11 accessions, while other causes of death, such as starvation, mechanical injury, and toxic chemicals accounted for 7 . In 315 accessions, no microbial pathogens were involved, and it was not possible to determine the etiologies in 29 accessions. New records of pathogens, hosts, and distribution are noted in the text.

\section{THE AUTHORS:}

Gerard M. Thomas is Staff Research Associate III, Department of Entomological Sciences, University of California, Berkeley.

George O. Poinar, Jr. is Lecturer and Associate Insect Pathologist, Department of Entomological Sciences, University of California, Berkeley. 


\section{Report of Diagnoses of Diseased Insects ${ }^{1}$ 1962-1972}

\section{INTRODUCTION}

THE LABORATORY FOR THE DIAGNOSIS of diseased insects was initiated in 1944 by the late Professor Edward A. Steinhaus and continued by him and Gordon A. Marsh to March, 1964. With the increased interest in the field of insect pathology and with diagnosis serving as its basis, the Berkeley diagnostic laboratory was continued along similar lines. This decision was also strengthened when it was learned that no other laboratory in the United States was performing a similar service for all insects (including agricultural, medical, and beneficial forms).

It must be emphasized that this work is a service, since there are no costs involved to the submitter and specimens are accepted from anywhere in the world. Aside from establishing new host and pathogen records, and helping graduate students to become better acquainted with a variety of diseaseproducing organisms, little use is made of most pathogens. This is unfortunate since some of these organisms undoubtedly have potential use in basic and practical studies. However, many of the microorganisms are kept in a culture collection and are available upon request. Unfortunately, the in- creasing costs involved in maintaining these cultures have forced us to ask a small fee for each culture.

During the first six years of the diagnostic service (from 1944 to 1950 ), a total of 575 accessions were received (Steinhaus, 1951). The second period, from 1951 to 1961 , covered 1,257 accessions (Steinhaus and Marsh, 1962). The present report covers 1,208 accessions received from 1962 to 1972 . During this period, the diagnostic service changed hands and some accessions were misplaced when previous diagnosticians moved to Irvine, California. Subsequent searches for these accessions failed and therefore it was impossible to include them in the present report. The missing accessions were: 1837, 1838, $1840,1842,1848,1867,1869,1872,1879$, $1880,1897,1899$, 1913, through 1922, 1947, 1948, 1950, 1965 through 1971, 1989, 1999, 2004, 2011, 2013, through 2015, 2018, 2019, 2030, 2033, 2034, 2044, 2048, 2050 through 2056, 2059, 2062, 2065 through 2067, 2072 through 2074, 2084, 2090 through 2093, 2099 through 2132,2135 through 2148,2150 through 2152, 2156 through 2161, 2167 through $2175,2182,2184,2189,2200,2201,2203$, $2205,2206,2214,2215,2219$, and 2228 .

\section{HOW TO SUBMIT SPECIMENS}

For quick and complete identification of pathogens, submitted specimens should contain relatively pure and viable pathogenic organisms. Thus, tim- ing is of extreme importance: it is preferable to submit living diseased insects or specimens freshly dead because the organism originally responsible for the

\footnotetext{
${ }^{1}$ Submitted for publication July 16, 1973.
} 
condition may break down and the action of secondary agents may mask the original etiology. This is one of the biggest problems confronting the diagnostician receiving specimens from long distances, and especially from warm climates. It is important, therefore, to submit specimens before general deterioration occurs. If this can't be done, some additional specimens should also be submitted in a preservative ( 70 per cent alcohol or 5 per cent formalin). Under ordinary circumstances, it is better not to use preservatives as the identification of bacteria and fungi (the two most common types of disease organisms encountered) depends on tests requiring their growth on various nutrient media.
When sending specimens, enclose them in a clean vial or pill box and include the following information.

1. Common and scientific name (including the family and author of the species) of the insect.

2. Date and locality of the collection.

3. Conditions under which the diseased material was found.

4. Any external signs or symptoms of the diseased specimens.

5 . Occurrence of other agents or factors, such as insecticides, extreme temperatures, crowding, etc. that might account for the condition.

Shipments made from outside the United States should be labelled "Dead biological specimens; no commercial value."

\section{DIAGNOSTIC PROCEDURE}

Every submitted shipment is assigned an accession number for our records and the sender is notified of this number when receipt of his shipment is acknowledged. A permanent accession card is made with information rceived from the submitter. This card and other special data sheets for specific groups of pathogens are shown in the previous published diagnostic report (Steinhaus and Marsh, 1962).

The specimens are thoroughly examined and sometimes, as in the case of a microsporidian infection or a bacteriosis caused by Bacillus thuriengensis, a diagnosis can almost be made by simply examining the affected tissues under the compound microscope, but, usually more time consuming tests have to be conducted before a diagnosis can be completed.

Eventually, the sender will receive a diagnostic report giving him the name of any pathogen found and other pertinent information. The sender is free to submit additional material or make any other inquiry regarding the diagnosis. The sender is entitled to use the information in publications and on the same token, our Department reserves the right to include the results of the examination in any of its publications. The Department also is entitled to use the diseased insects or isolated pathogens in any way it may desire.

\section{ARTHROPODA}

\section{Insecta}

\section{BLATTARIA}

\section{Blattidae}

Blattella germanica (L.)

2921-Two dead adult cockroaches which had been reared on dry food were submitted on October 13, 1969, by $\mathrm{R}$. Guttentag, Brooklyn, New York. Diagnosis: Mycosis caused by Aspergillus flavus. 
Leucophaea maderae (Fabricius)

2554-Several specimens of this roach were submitted by M. Martignoni, Corvallis, Oregon on January 13, 1965. All contained nematodes in their intestines and one contained bacteria in its hemocoel. Diagnosis: Nematodes belonging to Leidynema sp. and bacteria belonging to the following groups; A, Citrobacter group; B, Haffnia group, or possibly an Erwinia-like bacterium; C, Streptococcus faecalis.

\section{Periplaneta sp.}

2252_-Several specimens were received from D. R. A. Wharton, Natik, Massachusetts, on April 15, 1964. Some specimens were healthy; others were infected or dead. Dr. Wharton complained of a highly virulent infection in his laboratory colony. Extensive investigations were conducted, but no microbial pathogen was found. In one test, a strain of Serratia marcescens was found, but was not significant. Diagnosis: Undetermined.

\section{COLEOPTERA}

\section{Brentidae}

\section{Arrhenodes minitus (Drury)}

1900-One oak timberworm adult was submitted by R. D. Frye, Fargo, North Dakota, on July 20, 1962. The specimen was nearly hollow internally, with tissues shrunken against the inner surface of the exoskeleton and supporting a few miscellaneous gram negative bacterial rods. Diagnosis: Non-microbial.

1951-Several specimens were collected and submitted by R. D. Frye, Fargo, North Dakota, on September 25, 1962. Diagnosis: Mycosis caused by Beauveria bassiana.

\section{Carabidae}

Calosoma sp.

1957-One specimen was submitted by R. D. Frye, Fargo, North Dakota on
September 25, 1962. Diagnosis: Undetermined. Bacillus megaterium was isolated but was probably a secondary invader.

\section{Nechryopus savagei (Hope)}

2089-One adult of this predaceous ground beetle from Eastern Nigeria was submitted by A. Catley on July 30, 1969. The specimen showed a white matted mycelium along most of the sutures of the legs, coxal cavities, mandibles, antennal insertions, and around the ventral surface of the head at the insertion into the prosternum. Diagnosis: Mycosis caused by Metarrhizium anisopliae.

\section{Unidentified}

2987-One adult ground beetle, found while collecting ground beetles in Red Wood Park, Russian River, California, was submitted by S. Davidson on April 27, 1971. Diagnosis: Mycosis caused by Metarrhizium anisopliae.

\section{Cerambycidae}

\section{Megacyllene robiniae (Forst.)}

2394-Several specimens were submitted by C. J. Hay, Delaware, Ohio, on April 22, 1965. The larvae had been field-collected and reared in the laboratory on artificial media. All larvae on different types of media were affected. Symptoms of submitted larvae indicated a bacteriosis. Diagnosis: Bacterioses caused by Bacillus thuringiensis var. thuringiensis as the primary pathogen, and a secondary infection of Pseudomonas aeruginosa.

\section{Moneilema laevigata (Bland)}

2191-One specimen was submitted by A. Raske, Berkeley, California, on October 28, 1963. The submitted specimen was the only death in a colony of laboratory-reared $M$. laevigata. Diagnosis: Possible bacteriosis induced by sub-optimal environmental conditions affecting this particular specimen. The 
cadaver was badly deteriorated and contained a number of gram negative enteric bacteria.

\section{Moneilema sp.}

2224 Three pinned museum specimens were submitted by A. Raske, Berkeley, California, on February 3, 1964. Two specimens were $M$. laevigata, and the third was $M$. appressa LeConte. The specimens showed signs of fungus infection. Diagnosis: $M$. laevigata: both specimens were infected with Beauveria bassiana. The specimens of $M$. appressa harbored a secondary saprophytic fungus, Verticillium terrestre.

Monochamus sp. prob. notatus (Drury)

2642-One specimen in the larval stage was received from R. G. Dearborn, Orono, Maine, on June 28, 1966. This specimen was covered with white, cottony mycelium. Diagnosis: Mycosis Beauveria bassiana.

\section{Phymatodes nitidus (LeConte)}

1875-These specimens collected in Portola State Park, San Mateo County, California, were submitted by J. Powell and J. Chemsak on May 9, 1962. Diagnosis: Non-microbial; a Fusarium sp. was present on the surface of the specimens, but little or no penetration of tissues was evident.

\section{Tragidion sp.}

2087-These specimens were collected in Los Angeles County, California, and submitted by J. Chemsak on July 26, 1963. They had been starved for $4 \frac{1}{2}$ months. Diagnosis: General secondary bacteriosis, probably induced by the starved conditions.

\section{Unidentified specimen}

2338-An unidentified larva was submitted by D. Wood, Berkeley, California, on November 10, 1964. The single field-collected specimen had completely deteriorated into a fluid residue which was filled with a gram positive sporeforming bacterium. The bacterium was isolated in pure culture directly from the specimen. Diagnosis: Bacteriosis caused by Bacillus cereus.

\section{Cleridae}

\section{Cymatodera ovipennis (LeConte)}

2081-Several specimens were collected in Linden, California, and submitted by L. Falcon on July 11, 1963. It was possible that the area had been treated with an insecticide. Diagnosis: Non-microbial; no pathogens were found.

\section{Thanasimus dubius (Fab.)}

2416-Four diseased eggs of this Clerid were submitted by L. S. Pickard, Pineville, Louisiana, on June 14, 1965. Examination revealed the eggs were infected with a fungus. Diagnosis: $\mathrm{My}$ cosis caused by Aspergillus flavus.

\section{Chrysomelidae}

\section{Diabrotica undecimpunctata howardi (Barber)}

2721-Several adult specimens were received from J. C. Smith, Holland, Virginia, on January 19, 1967. All specimens showed signs of fungi, but examination revealed no pathogens. Diagnosis: probably non-microbial or, if microbial, the primary agent had been obscured by saprophytic fungi such as Rhizopus nigricans, Aspergillus ochraceous and a Penicillium sp.

2773-Several larvae of the southern corn rootworm were received from $R$. B. Chalfant, Tifton, Georgia, on September 19,1967 . The specimens were soft and white in appearance. Diagnosis: Microsporidiosis caused by a Nosema sp.

2860-Smears made from larval and adult stages of this beetle were fixed in methyl alcohol for 2 minutes and submitted on September 20, 1968, by R. B. 
Chalfant, Tifton, Georgia, with the request for confirmation of a possible Nosema infection. Thorough examination of the smears by staff members failed to confirm a Nosema infection. Diagnosis: Not possible from the submitted material.

\section{Oulema melanopa (L.)}

2175-Several laboratory reared specimens of the cereal leaf beetle were submitted by T. Castro, East Lansing, Michigan, on October 2, 1963. The specimens were dessicated. Diagnosis: General bacterioses, consisting of gram negative and gram positive strains, with a Proteus sp. being dominant. No groups containing entomogenous pathogens were found.

2216-Several laboratory reared specimens were submitted by T. $R$. Castro, East Lansing, Michigan, on December 31, 1963. Diagnosis: Bacterioses. Mixed strains of gram negative rods and gram positive cocci present. One strain, Aerobacter aerogenes, predominated.

2349_-Several adults were collected in the field and submitted by C. F. Koval, Madison, Wisconsin, on January 18, 1965. Diagnosis: Mycosis: Beauveria bassiana.

2413-Three larvae were submitted by M. S. Gomulinski, East Lansing, Michigan, on June 2, 1965. Of the four specimens, one suffered a bacterioses and the rest showed no microbial etiology. Diagnosis: Bacteriosis caused by Pseudomonas aeruginosa.

2485-Several specimens were submitted by M. Gomulinski, East Lansing, Michigan, on September 30, 1965. They had been removed from field over-wintering cages and subsequently died in the lab. Diagnosis: General bacterial deterioration, primary etiology obscured.

2489-One specimen "obtained from a graduate experiment" was submitted by M. Gomulinski, East Lansing, Michigan, on October 7, 1965. Diagnosis: General bacterial deterioration; no primary pathogens.

2809-Several diseased cereal leaf beetle larvae from a laboratory culture were submitted by S. G. Wellso, East Lansing, Michigan, on February 26, 1968. The larvae were dying in the last larval instar, and exhibited symptoms of a bacteriosis. Diagnosis: Bacteriosis caused by Streptococcus faecalis var. liquefaciens and a non-chromogenic strain of Serratia marcescens.

2997-Three larvae of the cereal leaf beetle which died in the laboratory culture of S. G. Wellso, East Lansing, Michigan, were submitted to the Diagnostic Service on June 21, 1971. Diagnosis: Non-microbial.

3014-Fecal debris from old adults of the cereal leaf beetle was submitted by S. G. Wellso, East Lansing, Michigan, on September 15, 1971, to check for the presence of pathogens. Diagnosis: No pathogens found.

\section{Pagria signata (Mots)}

2357_-Several adult specimens were submitted by S. Rao, Kuala Lampur, Malaya, on February 15, 1965. Diagnosis: Mycosis caused by Beauveria tenella.

\section{Coccinellidae}

\section{Hyperaspis lateralis Muls.}

2938_-Several adult coccinellids preserved in 70 per cent ethyl alcohol, some dry fungus material in a vial scraped from the beetles, and a microscope slide of fungus material were submitted by K. Hagen, Berkeley, on February 16, 1970. The preserved specimens showed no signs of a primary microbial etiology. The dry fungus material was a saprophytic Aspergillus sp. nr. ochraceus and the microscope slide contained another saprophyte, Rhizopus nigricans. Diagnosis: Non-microbial. 


\section{Curculionidae}

Aramminchus (Otiorhynchus) cribicollis Gyll.

2752-Several diseased larvae were submitted by C. T. Buchelos, Patras, Greece, on May 20, 1967. These specimens were brown and shriveled. Diagnosis: Bacteriosis caused by a non-chromogenic strain of Serratia marcescens. A secondary bacterium, Aerobacter grp. $\mathrm{C}$, was also present.

\section{Cecyropa sp.}

1993-Two adult weevils, showing external signs of a possible fungus infection were submitted by G. A. H. Helson, Wellington, New Zealand, on December 13, 1962. Diagnosis: Probably non-microbial. The fungus present was a Fusarium sp. which had not penetrated the tissues. A check of the internal tissues for the presence of major groups of entomogenous pathogens was negative.

\section{Cosmopolites sordidus (Germar)}

2377-One larva of the banana root weevil was submitted by F. Lara, Limon, Costa Rica, on March 31, 1965. The specimen was collected in La Estrella Valley, Costa Rica. Diagnosis: Bacterioses, Pseudomonas aeruginosa and Septococcus sp. Probably invaded the insect through wounds.

\section{Cossonus piniphilus Boheman}

2006-Many specimens of this weevil apparently infected with a Hirsutella sp. were discovered by C. W. O'Brien in a Bishop pine tree near Inverness, California. Additional specimens were collected from the same tree; some showed synnematia, others did not. Diagnosis: Mycoses caused by Synnematium jonesii and Beauveria bassiana.

\section{Cylas formicalius (Fab.)}

2728-A few adult weevils were received from H. Katayama, Sasayama,
Hyogo-ken, Japan, on February 21, 1967. Diagnosis: Mycosis: Beauveria bassiana.

2734-Several adult specimens of the sweet potato weevil were received from H. Katayama, Sasayama, Hyogo, Japan, on March 24, 1967. They were collected on the Ryuku Islands, Okinawa. Diagnosis: Mycosis: Metarrhizium anisolpiae.

\section{Cylindrocopturus eatoni Buchanan}

2190-Fifteen larvae of this weevil were collected in Groveland, Tuolumne County, California, on Ponderosa pine by R. E. Stevens on October 5, 1963. Examination and microbial analysis produced no pathogenic microorganisms. Diagnosis: Non-microbial.

\section{Graphognathus sp.}

3007-Four grubs found dead in the field were received on August 25, 1971 from R. Ahmad, Bariloche, Argentina. The grubs were brown and deteriorating. Diagnosis: Non-microbial.

3021-A few brown and deteriorating grubs of this weevil were submitted by R. Ahmad, Bariloche, Argentina, on October 12, 1971. Diagnosis: Non-microbial.

\section{Hypera brunneipennis (Boheman)}

2943-Several larval specimens of this weevil were collected from alfalfa fields near Woodland, California, by C. S. Koehler on March 23, 1970. The affected specimens were sluggish, and exhibited abnormal pink coloration. Diagnosis: Mycoses caused mainly by Beauveria bassiana. One specimen showed infection by an Entomophthora sp.

\section{Hypera postica (Gyll.)}

2068-A single specimen, field-collected and reared in the laboratory was one of twelve that died from an original 
collection of 600 . Submitted by W. R. Cothran, Ithaca, New York, on June 12, 1963. Diagnosis: Mycosis caused by Beauveria bassiana.

2095-Several specimens were submitted by W. H. Ziener; Amherst, Massachusetts, on August 5, 1963. The weevils were from laboratory cultures and the field. Diagnosis: The laboratory culture suffered a mycosis caused by Beauveria bassiana. The field-collected specimens' cause of death was not determined.

2266-Several field-collected larvae were submitted by C. F. Lagace, Albany, California, on June 2, 1964. The specimens were filled with bacteria which proved to be secondary saprophytic invaders. Diagnosis: Undetermined, possibly non-microbial.

2374, 2376, 2381, 2382, 2383, 2390These specimens were collected by $R$. van den Bosch in Lebanon, and submitted by G. Finney on March 29, 1965. Diagnosis: Non-microbial in some, and mycosis caused by Beauveria bassiana in others.

2286-Several larvae were collected in the field in Siskiyou County, California, by C. S. Koehler; on July 3, 1964. Diagnosis: Probably non-microbial with secondary bacterioses - Streptococcus sp. and Aerobacter grp. C.

2388, 2397-Several specimens collected in Chtaura, Lebanon, by $R$. van den Bosch, on March 31 and April 15, 1965 , were submitted by G. Finney on April 12 and April 27, 1965. Diagnosis: Mycosis caused by Beauveria bassiana, parasitism and bacteriosis.

2448-On August 3, 1965, R. Hedlund, Lexington, Kentucky, submitted several specimens suffering from a fungus infection. Diagnosis: Mycosis caused by Beauveria bassiana.

2611-Several dry specimens and a fungus culture isolated from them were submitted by R. B. Subba Rao, New
Delhi, India, on May 10, 1966. Diagnosis: The fungus was a Chalaropsis sp. The specimens suffered a bacterioses caused by Bacillus cereus and Streptococcus faecalis.

2748-Several diseased larvae were submitted by C. Koehler from Porterville, California, on April 28, 1967. Diagnosis: Mycosis: a species of Spicaria, or Paecilomyces.

2858-Three alfalfa weevil larvae which had been stored in the refrigerator were submitted by G. Finney, Berkeley, California, August 13, 1968. A routine pathogen check was requested. Examination revealed no frank microbial infection, and microbial analysis yielded one isolate of a saprophytic bacterium, Aerobacter sp. Diagnosis: Cause of death was probably nonmicrobial.

2900-Many dead larvae were submitted by R. van den Bosch from Clampino (Rome), Italy, on July 1, 1969. Examination of specimens indicated a general secondary bacterioses. Diagnosis: Secondary bacteriosis caused by Streptococcus sp. and Aerobacter grp. C., which indicates that some other predisposing factors were probably operating in the environment.

2985-R. K. Smith, Urbana, Illinois, submitted six adult weevils on April 23, 1971. The weevils had been reared in the laboratory in plexiglass tubes, where the humidity was high. Diagnosis: Mycosis caused by Beauveria bassiana.

\section{Lissorhopturus oryzophilus (Kuschel)}

2847-Several adult rice water weevils were submitted by J. E. Lauck, Davis, California, on July 16, 1968. These specimens had been field-collected and then held in the laboratory at $43^{\circ} \mathrm{F}$. Examination showed them to be hollow internally, with all tissues dried and shrunken against the body walls. Tissues showed evidence of some septate 


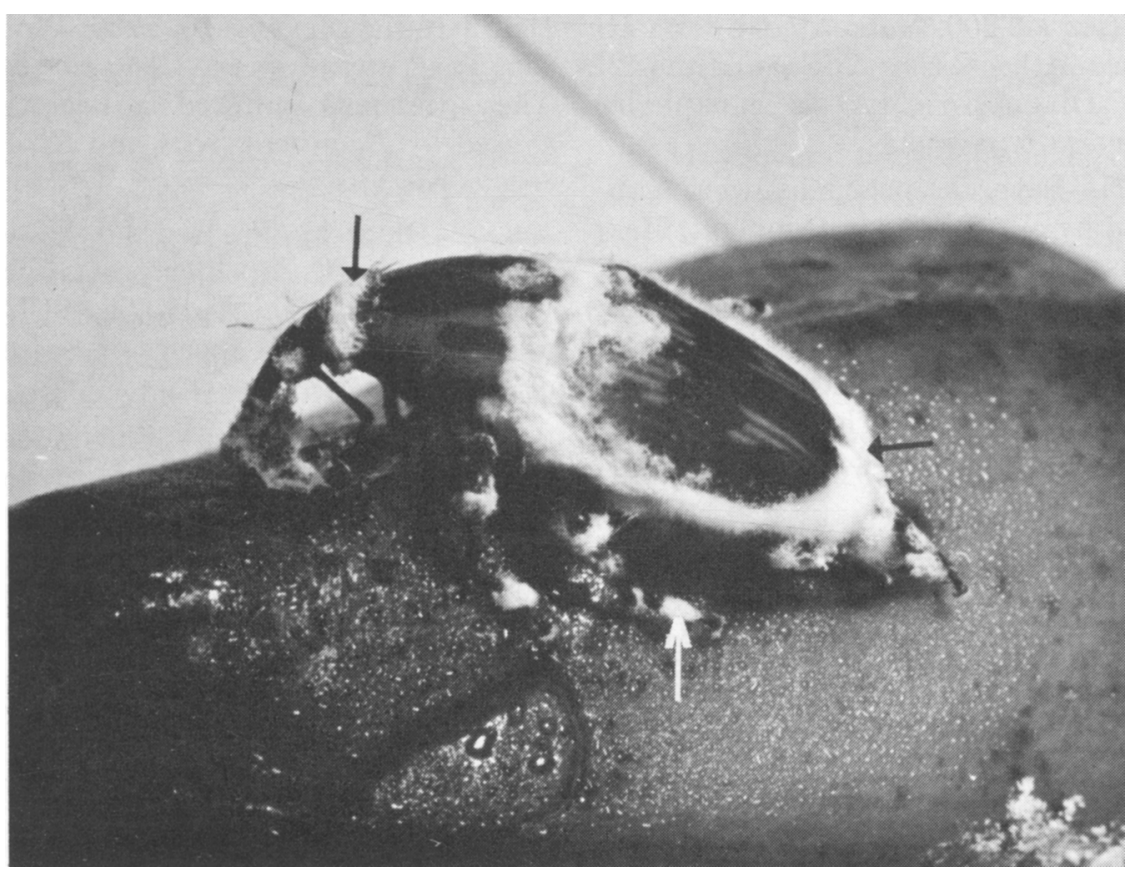

Fig 1. Acc. No. 2458: Adult banana weevil (Metamasius hemipterus) infected with Beauveria bassiana. Note fungal mycelium emerging from inter-segmental areas (arrows). Magnification $=5 \mathrm{X}$.

mycelium, but microbial analysis produced only a few saprophytic fungi. Diagnosis: Non-microbial.

2853-On August 8, 1968, one adult weevil was received from J. Lauck, Davis California. This specimen was found in the field and exhibited a possible mycasis. Upon examination, however, the fungus was found to be a Dematiaceae in the saprophytic genus Bactrodesmiella. Diagnosis: Probably non-microbial.

2909_-One dead adult was submitted by J. Knabke, Davis, California. This specimen was from a laboratory culture and contained mycelium on its surface. Examination showed the fungus to be one of the aquatic Phycomycetes in the Peronosporales. Diagnosis: Non-microbial.

Metamasius hemipterus (L.)

2458-One weevil found on a banana in a grocery store was submitted by $\mathrm{J}$. Powell, Berkeley, California, on August 16, 1965. Diagnosis: Mycosis caused by Beauveria bassiana. New record. (Fig. 1.)

Microlarinus lareynii (Jaqueline du Val)

2505-Several dead specimens of the puncture vine weevil, collected in Modesto, California, on September 16, 1965, were submitted by J. Hamai, Berkeley, California. The specimens were beginning to deteriorate, and showed signs of a bacterial infection. Diagnosis: Bacterioses caused by Streptococcus faecalis, and a secondary Aerobacter sp.

Nemocestes incomptus (Horn)

3031-A few adults of this root weevil, covered with mycelium, were submitted by W. T. Cram, Vancouver, Brit- 
ish Columbia, Canada, on November 19, 1971. The weevils were from a laboratory culture where the disease had become epizootic in proportions. Diagnosis: Mycosis caused by Beauveria bassiana.

\section{Pantomorus godmani (Haw.)}

2384-Two adults of the Fuller rose beetle were submitted by $\mathrm{Y}$. Tanada, Berkeley, California, on April 9, 1965. They were collected at the Kahua Ranch, Waimea-Kohala, Hawaii, on March 24, 1965. Diagnosis: Mycosis caused by Beauveria bassiana.

\section{Pantomorus sp.}

3012-A few adults of the whitefringed beetle, submitted on September 8, 1971 by R. Ahmad, Bariloche, Argentina, were infected with a fungus. Diagnosis: Mycosis caused by Beauveria bassiana.

3020 - A few specimens of the whitefringed weevil were received from $R$. Ahmad, Bariloche, Argentina, October 12, 1971. The specimens were infected with nematodes. Diagnosis: Probably a Neoaplectana sp.

\section{Pissodes terminalis (Hopk.)}

2009-One larva of the lodgepole terminal weevil collected in Truckee, California, was submitted by R. W. Stark and D. L. Wood of Berkeley, California, on February 7, 1963. Diagnosis: Nonmicrobial, death due to mechanical injury.

2760-Several larvae were submitted by A. Cameron, Berkeley, California, on July 21, 1967, with a request for examination for possible pathogens. Diagnosis: Non-microbial.

2861-Three larvae were brought in for a routine pathogen check by $A$. Cameron, Berkeley, California, on September 27, 1968. Thorough examination revealed no primary pathogens present. Diagnosis: Non-microbial.
2894 - Three dead larvae were dissected out of lodgepole pine terminals by A. Cameron, Berkeley, California, and submitted for a routine pathogen check on June 2, 1969. Thorough examination showed no microbial etiology in two specimens, but the third was filled with monorefringent protein crystals similar to virus polyhedra. Electron microscope studies revealed a configuration similar to that of a cytoplasmic polyhedrosis virus inclusion body, but dissolution in hydroxide was so rapid that confirmation was not possible. Diagnosis: Non-microbial in two specimens. A possible virus etiology in one specimen, but sufficient material was not available for confirmation. Additional material was requested.

2898-As requested above, additional specimens of this weevil were submitted by A. Cameron on June 27, 1969. Of the four larvae brought in, one showed the protienaceous crystals, but electron microscope study failed to show virus particles. Diagnosis: Probably non-microbial.

2905-On July 21, 1969, five more specimens of this weevil were received from A. Cameron. Of the five dead larvae, four were negative for a microbial etiology, and one cadaver was filled with the monorefringent protein crystals. However, again electron microscopy failed to confirm a virus nature for these crystals. Diagnosis: Probably non-microbial.

2906-Three dead weevil larvae and one dead adult were submitted by $\mathrm{A}$. Cameron on August 7, 1969. All were thoroughly examined, but no microbial etiology was found. Diagnosis: Nonmicrobial.

2947-On April 20, 1970, A. Cameron submitted four dead larvae which had been dissected out of lodgepole pine tips. These tips were field-collected and held in laboratory refrigeration for overwintering. A routine-check for 
pathogens was negative; however, two specimens contained the protein crystals found previously. Electron microscope study again failed to show a virus nature for these crystals. Diagnosis: Probably non-microbial.

2948 - Five additional specimens from the same overwintering material mentioned above were submitted by $\mathrm{A}$. Cameron on April 22, 1970, for a routine pathogen check. No pathogens were found, but two specimens contained the protein crystals found previously. However, electron microscope study failed to show a virus nature for these crystals. Diagnosis: Probably non-microbial.

2950_Seven more specimens were submitted by A. Cameron from his laboratory overwintering stock. Routine pathological examination and microbial analysis revealed no primary microbial etiology. Diagnosis: Non-microbial.

\section{Pissodes yosemite (Hopkins)}

2005B-Four larvae of the Yosemite bark weevil were collected in Groveland, California, and submitted by $\mathrm{R}$. E. Stevens, Berkeley, California, on January 28, 1963. Diagnosis: Undetermined.

2204-Four larval specimens were collected in Groveland, California, and submitted by R. E. Stevens, Berkeley, California, on December 6, 1963. The specimens had been collected in September and stored at $40^{\circ} \mathrm{F}$ before submittal. Diagnosis: Mycosis caused by Beauveria bassiana.

\section{Rhabdoscelus asperipennis (Fairmaire)}

2199_Several diseased larvae of this weevil were submitted by F. A. Bianchi, Pacific Trust Territory, Koror, Caroline Islands, on November 20, 1963. Diagnosis: Mycosis caused by Metarrhizium anisopliae.

Smicronyx sp. nr. albovariegatus Faust 2245-Several diseased larvae were submitted by V. P. Rao, Bangalore, India, on March 26, 1964. The submitted specimens were collected in Ranebennur, Dharwar District, Mysore State, India, extracted from witch weed galls, and placed in soil in the laboratory for pupation. About 50 per cent of the population became infected with a fungus. Diagnosis: Mycosis caused by Beauveria bassiana.

\section{Stenopelmus refinasus Gyllenhal}

2430-Several adult weevils were submitted by A. A. Grigarick, Davis, California, on July 8, 1965 . These weevils are semi-aquatic and the submitted specimens had been reared in the laboratory. Diagnosis: Mycosis caused by Beauveria bassiana.

\section{Unidentified weevil}

3003-One dead weevil larva was submitted by R. Ahmad, Bariloche, Argentina, on August 6, 1971. Examination and microbial analysis revealed no microbial pathogens. Diagnosis: Nonmicrobial.

\section{Dermestidae}

\section{Trogoderma glabrum (Herbst)}

2036-Four laboratory reared specimens were submitted by W. A. Burkholder, Madison, Wisconsin, on March 27, 1963. Diagnosis: Protozoan infection caused by Mattesia dispora.

\section{Trogoderma inclusum LeConte}

2035-Four laboratory reared larval specimens were submitted by W. E. Burkholder, Madison, Wisconsin, on March 27, 1963. Diagnosis: Protozoan infection caused by Mattesia dispora.

2779_-Several diseased carpet beetle larvae were submitted by $\mathrm{Y}$. Carmi, Jaffa, Israel, with the request for identification of the protozoan. Diagnosis: Coccidiosis: Adelina sp. (Fig. 2.) 

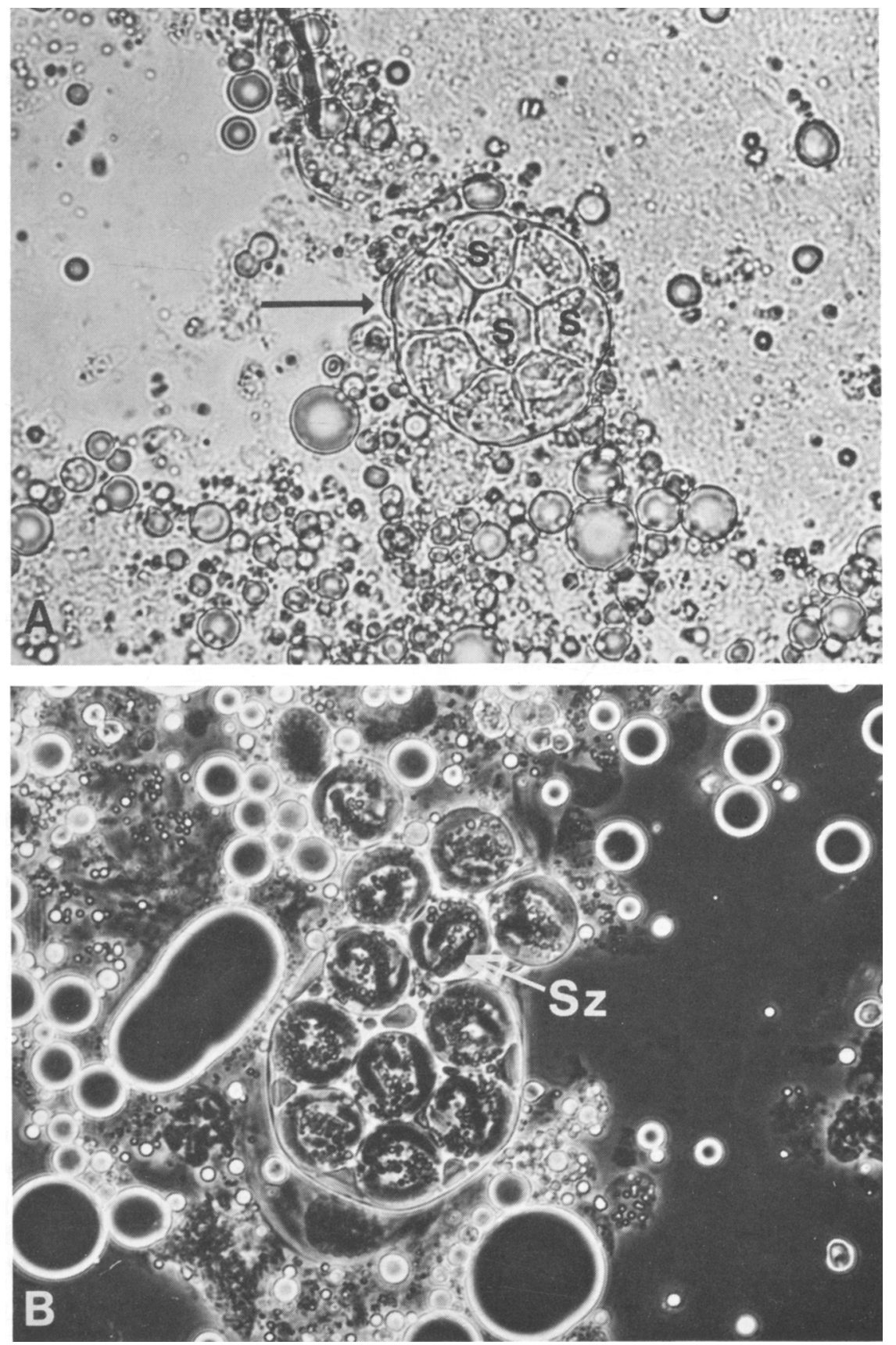

Fig. 2. Acc. No. 2779: Fat-body tissue smears from Trogoderma inclusum infected with an Adelina sp. A: Intact oöcyst (arrow) containing developing sporoblasts (S). Magnification = 800X. B: Phase-contrast micrograph of a ruptured oöcyst showing sporoblasts containing developing sporozoites (Sz.) Magnification $=800 \mathrm{X}$. 


\section{Elateridae}

Unidentified wireworms

3005-Several dead click beetle larvae were submitted by E. Omi, Berkeley, California, on August 8, 1971. The specimens were from a laboratory culture. Examination revealed a mixture of secondary saprophytic bacteria. Diagnosis: Probably non-microbial.

\section{Eumolpidae}

\section{Colaspis hypochlora Lefebre}

2817-One adult beetle, covered with a fungus, was submitted by C. S. Stephens, Changuinola, Panama, on April 2, 1968. The specimen had been collected from banana on the Caribbean Coast near the Costa Rican border. Diagnosis: Mycosis caused by Beauveria bassiana.

\section{Halticidae}

\section{Haltica carduorum Guer.-Men.}

2927-Many larvae stuck to thistle leaves by a fungus were submitted by L. Anderson, Palisade, Colorado, on November 26, 1969. Diagnosis: Mycosis caused by Beauveria bassiana.

\section{Melandryidae}

Unidentified (Melandryid beetle)

1904-One dead larva found under the bark of a fallen oak tree was submitted by R. D. Frye, Fargo, North Dakota, on July 20, 1962. The submitted specimen was dry and hollow except for some thin mycelium. No evidence of a primary pathogen was found. The fungus was isolated in culture and identified as a common wood inhabiting saprophyte, Trichoderma koningi. Diagnosis: Probably non-microbial

\section{Meloidae}

\section{Epicauta cinerea Forst.}

2727-A preserved mid-gut and two histological slides from a supposedly diseased blister beetle were submitted by P. H. Thompson, Brunswick, New Jersey, on February 16, 1967, along with a description of an apparent disease condition. Diagnosis: No microbial disease present. The condition described by the submitter was obviously a misinterpretation of artifacts produced in fixing and sectioning the submitted material.

\section{Melolonthidae}

Psilopholis vestita (Sharp)

2510 - One somewhat deteriorated specimen of this grub was submitted by B. Sripathi Rao, Kuala Lumpur, Malaysia, on October 28, 1965. This grub is a serious pest of rubber trees and appeared to be suffering from a fungus infection. Diagnosis: Mycosis caused by Metarrhizium anisopliae.

\section{Ostomidae}

Temnochila virescens ssp. chlorodia (Mann.)

2696 - Several larval specimens which had been reared in the laboratory on boiled Galleria melonella (Linnaeus) larvae were submitted by T. O'Connell, Albany, California, on October 6, 1966. Examination indicated a bacterial infection. Diagnosis: Bacteriosis caused by Bacillus cereus.

\section{Scarabaeidae}

\section{Anomala expansa (Bates)}

1941-A fungus culture isolated from $A$. expansa was submitted on September 7, 1962 by Wen-Yi-Cheng, Tainan, Taiwan, for identification. Diagnosis: Mycosis caused by Metarrhizium anisopliae. This was the first record of this fungus from this host in Taiwan.

\section{Bothynus gibbosus (DeGeer)}

2920-Seven dead adults of the carrot beetle were submitted by D. G. Bottrell, Lubbock, Texas on October 2, 
1969. These were collected from sunflower and pigweed and appeared to be infected with a fungus. Diagnosis: $\mathrm{My}-$ cosis caused by Metarrhizium anisopliae.

\section{Copris incertus Say}

2574 - Two specimens in 70 per cent ETOH and one dry specimen were submitted by G. F. Bornemissza, Kamuela, Hawaii; they were collected in January and February 1966 in Waikii, Hilo and Puako, Hawaii. Diagnosis: Two specimens collected at Waikii: non-microbial. They had a surface growth of what appeared to be some algae. Dissection and examination showed no internal mycosis, nor any other microbial etiology. Preservation in 70 per cent ETOH prevented cultivation of microorganisms. One specimen collected at Puako: mycosis caused by Beauveria bassiana.

\section{Costelytra zealandica (White)}

1908-Several specimens of this grass grub were submitted by G. A. H. Helson, Wellington, New Zealand, on July 24, 1962. Insecticide treatments had been carried out 1 year previously. At that time, full-strength DDT had been used. Diagnosis: Non-microbial.

1935-Two specimens of this grass grub collected in Outram Otago, New Zealand, were submitted on August 28, 1962 , by G. A. H. Helson. The specimens were part of a lot collected in the field and reared in the laboratory at Wellington. After 5 weeks, all specimens developed a fungus. Diagnosis: Mycosis caused by Metarrhizium anisopliae. Probably a new record for this host.

\section{Cyclocephala signaticollis Burmeiter}

2655-Two specimens collected in the field and reared in the laboratory were submitted by L. E. San Martin, Balcarce, Argentina, on July 26, 1966. Diagnosis: Undetermined. The submitted specimens had been completely destroyed by saprophytic mites, making a diagnosis impossible.

\section{Eremobothynus cornutus (Stnhl.)}

3004-Two black and shriveled dead grubs were submitted by Rafiq Ahmad, Bariloche, Argentina, on August 6, 1971. Examination showed an abundance of bacterial spores. Diagnosis: Bacteriosis by the potential pathogen Bacillus cereus.

\section{Heterogomphus pauson (Perty)}

2654-Two larvae were received from L. E. San Martin, Balcarce, Argentina, on July 26, 1966. The cadavers were yellow, tinged with violet, and mummified. Diagnosis: Mycosis caused by a Cordyceps sp.

\section{Holotrichia sp.}

2230 - A fungus culture isolated from cockchafer grubs was submitted by $\mathrm{P}$. Somasekhar, Mysore State, India, on February 11, 1964. Diagnosis: Mycosis caused by Metarrhizium anisopliae.

\section{Pericoptes truncatus Fab.}

1994 - Some specimens of this sand scarab were submitted by G. A. H. Helson, Wellington, New Zealand, on December 13, 1962. Mr. Helson suggested infection by Serratia marcescens. Diagnosis: Undetermined. The presence of $S$. marcescens was not confirmed. The predominant bacterium isolated from the specimens was a strain of Escherichia freundii.

1995-One additional specimen of this sand scarab was submitted by G. A. H. Helson, Wellington, New Zealand, on December 5, 1962. Diagnosis: Mycosis caused by Metarrhizium anisopliae.

\section{Polyphylla sp.}

2829-A few larvae of this scarab were received from J. Onsager, Yakima, Washington, on May 20, 1968. The 


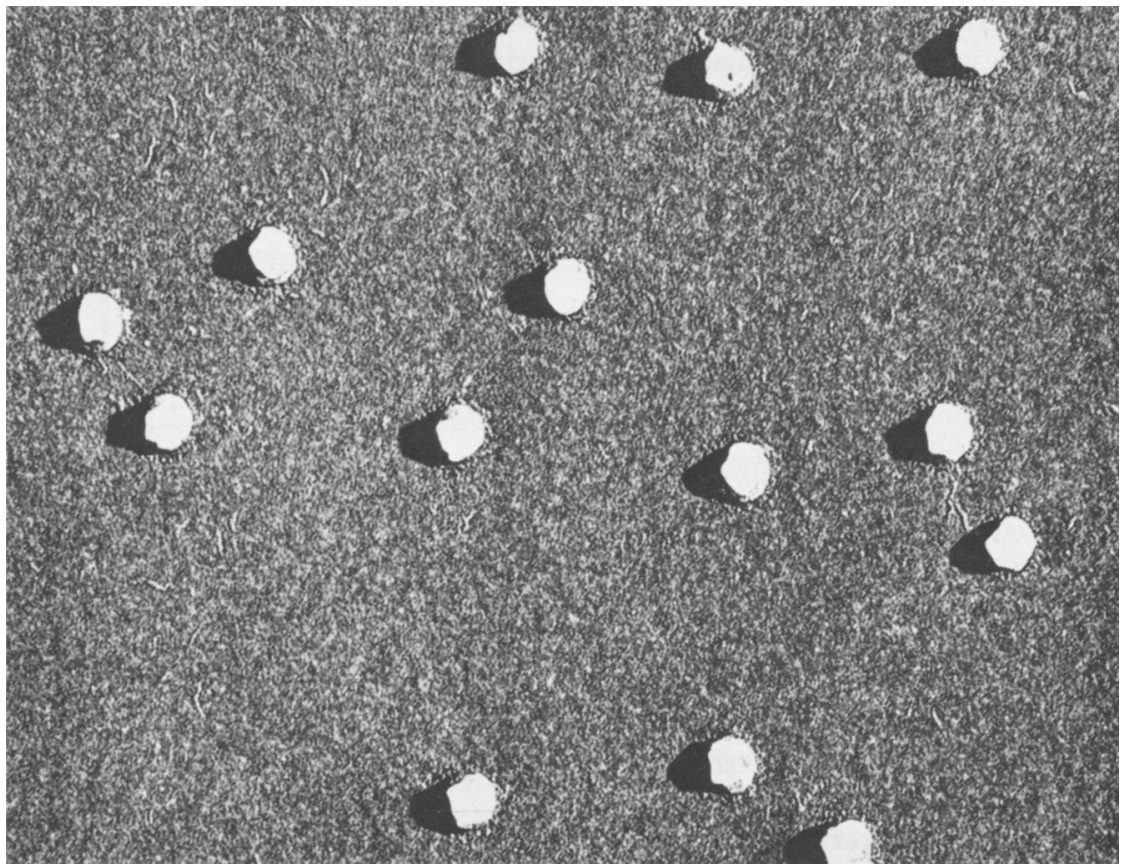

Fig. 3. Acc. No. 1976: Electron micrograph of virus particles of SIV (Sericesthis Iridescent Virus) from Sericesthis pruinosa. Magnification $=35,000 \mathrm{X}$.

specimens were infected by a Cordyceps sp., and specific identification of this fungus was requested. Diagnosis: $\mathrm{My}-$ cosis, caused by a Cordyceps sp. The stroma on the submitted specimens was immature, and attempts to culture the fungus and force maturity were unsuccessful; thus, specific identification was not possible.

\section{Sericesthis pruinosa (Dalman)}

1976-One larva of the pruinose scarab was received from R. J. Roberts, New South Wales, Australia, on October 29 , 1962. Studies on this specimen, transmission of hemolymph to Galleria melonella larvae, and studies of the resulting material showed the presence of an icosohedral virus of the TIV type. This was the first record of this virus, which eventually became known as the Sericesthis Iridescent Virus (SIV). Diagnosis: Virosis caused by SIV. (Fig. 3.)

\section{Strategus antaeus (Drury)}

2917-Two deteriorated and putred grubs were received from W. G. Genung, Belle Glade, Florida, on September 23, 1969. Diagnosis: Bacterioses caused by the potential pathogens Bacillus cereus and Serratia marcescens.

\section{Unidentified specimen}

1958-One field-collected specimen which died in a vial, was submitted by R. D. Frye, Fargo, North Dakota. When received, the specimen was shriveled and dessicated with no external signs of a frank microbial infection. Diagnosis: Undetermined. Microbial analysis produced only a few gram negative small rods, which were present in very low numbers. No known pathogenic microorganisms were found.

1964-One specimen submitted by G. A. H. Helson, Wellington, New Zealand, on October 9, 1962. Diagnosis: Undetermined. No known insect patho- 
gens were noted or isolated from this single specimen.

\section{Unidentified scarab larva}

2038-This specimen was collected and submitted by E. H. Wollerman, Delaware, Ohio, on April 3, 1963. Diagnosis: Mycosis caused by Cordyceps sp. The stroma on the submitted specimen was immature, and attempts to force maturity were unsuccessful. The species was either C. melolonthae or C. ravenelli.

\section{Xylopyctes sp.}

2310_Several specimens were submitted by M. J. MacQuillan, Honiara, British Solomon Islands, on August 31, 1964. They had been preserved in alcohol, and a diagnosis was not possible. Diagnosis: Undetermined.

\section{Scolytidae}

\section{Dendroctonus brevicomis (LeConte)}

2606-Several larvae were received from W. Bedard, Berkeley, California, on May 2, 1966. Diagnosis: Probably non-microbial.

2653-Ten adults were received from D. Wood, Berkeley, California, on July 25, 1966. Diagnosis: Isaria species which were non-pathogenic in tests against Galleria melonella; therefore, the fungus may be a saprophyte and have obscured the primary cause of death.

2683 - A yeast culture was received from J. Ball, Berkeley, California, on September 16, 1966. Diagnosis: The yeast was tested against Galleria larvae for pathogenicity by intra-hemocoelic and per-os injections. The results of both tests were negative. We concluded that the yeast in question is not a pathogen, at least not under the conditions of these tests.

\section{Dendroctonus frontalis (Zimm.)}

2021-Some 21 adults of the southern pine beetle were received from J. C.
Moser, Alexandria, Louisiana, on March 1,1963 . These beetles died about 5 days after emerging from a bolt of shortleaf pine. Diagnosis: Mycosis, caused by Aspergillus flavus. Probably a new host record for this fungus.

2365-Several larvae were submitted by L. S. Pickard, Pineville, Louisiana, on March 11, 1965. They had been kept at $40^{\circ} \mathrm{F}$ for 14 days prior to this. Two strains of yeast were isolated. Infectivity tests with these yeasts against Galleria melonella gave negative results. Diagnosis: Probably non-microbial.

2711-Eight bacterial cultures isolated from the southern pine beetle were received from G. E. Moore, Research Triangle Park, North Carolina, on December 14, 1966. Diagnosis: The cultures were identified as follows: one culture of Bacillus thuringiensis var. thuringiensis, two cultures of Aerobacter grp. C, two cultures of an Alcaligenes sp., two cultures of Pseudomonas aeruginosa, and one culture of a gram negative, pleomorphic, non-spore forming, non-flagellate, non-fermentative rod, which was not identified.

2735-On March 28, 1967, eight additional bacterial cultures isolated from the southern pine beetle were received from G. A. Moore, Research Triangle Park, North Carolina. Diagnosis: All eight culture tubes contained Pseudomonas aeruginosa, and one also contained Alcaligenes faecalis.

\section{Ips calligraphus Germ.}

2313-Twelve larvae were submitted by J. C. Moser, Alexandria, Louisiana. The larvae were collected August 21, 1964 , and stored at $40^{\circ} \mathrm{F}$ until September 1,1964 . Only some secondary bacteria were found. Diagnosis: Probably non-microbial.

\section{Scolytus ventralis LeConte}

2305-Several larvae of the fir engraver were submitted by $R$. Stark, 
Berkeley, California. These were collected at Blodgett Forest, Eldorado County, California, on July 29, 1964. The larvae were in pupal cells which were completely filled with a dense mycelial growth of an Oedocephalum sp. This fungus belongs to a group normally considered saprophytic and was probably a saprophytic secondary invader, rather than a primary pathogen. Diagnosis: Probably non-microbial, or if microbial, then the primary etiology has been masked by the invasion of the secondary saprophytic fungus.

2682-Several larvae were received from M. Ashraf, Pullman, Washington, on September 9, 1966. Microbial analysis produced a pathogenic fungus. Diagnosis: Mycosis: Aspergillus flavius.

\section{Tenebrionidae}

\section{Coelocnemis punctata LeConte}

2862-Three preserved adults were submitted by J. Doyen, Berkeley, California, on October 1,1968 . These specimens were from a stock culture in which dead adults exhibited black nodules in the tissues. Examination of the fixed tissues showed a microsporidian infection. Diagnosis: Microsporidiosis, probably Nosema sp.

\section{Coelocnemis sp.}

2799-Five larvae were submitted from a laboratory culture by J. Doyen on January 15, 1968. Diagnosis: Nonmicrobial. A thorough examination revealed no pathogens. Microbial analysis yielded only Rhizopus nigricans and an Aerobacter sp.

\section{Tenebrio molitor Linnaeus}

2952-One adult and several pupae were submitted from a laboratory culture by B. Hammoca, Berkeley, California, on June 3, 1970. Examination and microbial analysis showed no pathogens present. The cadavers had been invaded by saprophytic bacteria, suggesting that the primary cause of death was other than microbial. Diagnosis: Non-microbial.

\section{Tribolium anaphae Hint.}

2589-A sample of dead larvae from a laboratory culture of this flour beetle was submitted by M. Hoy, Berkeley, California, on March 31, 1966. Mortality in the laboratory culture was significant. Diagnosis: Microsporidiosis caused by Nosema whitei.

\section{Tribolium castaneum (Herbst)}

2022 - A large number of laboratory reared larvae were submitted by $\mathrm{P}$. S. Tyler, Slough, Bucks, England, on March 1, 1963. Two protozoans were isolated. Diagnosis: Triboliocystis garnhami and Nosema whitei.

2043 et al.-From April 15, 1963, to March 12, 1969, numerous specimens of the red flour beetle were submitted for routine pathogen checks. These included some 99 accessions (see numbers below) from the Department of Genetics, University of California, Berkeley, California. The source material was from stock and experimental cultures of various genetic strains. Diagnoses: Protozoan infections were found in 59 accessions and consisted of the following organisms: Farinocystis tribolii (8); and Nosema whitei (51). Bacteriosis caused by Streptococcus faecalis was found in eight accessions. Thirty-seven accessions were non-microbial. Accession numbers: 2043, 2192, 2196, 2208, 2226, 2232, 2236, 2238, 2239, 2263, 2264, $2283,2333,2362,2373,2409,2410,2412$, $2439,2449,2453,2470,2471,2472,2477$, $2482,2491,2492,2496,2498,2503,2506$, $2513,2518,2519,2522,2524,2525,2530$, 2535, 2536, 2538, 2539, 2541, 2543, 2548, $2550,2552,2558,2561,2569,2573,2575$, $2583,2588,2591,2599,2601,2604,2607$, $2618,2619,2621,2629,2634,2643,2644$, $2646,2661,2670,2673,2679,2703,2709$, $2718,2722,2726,2730,2739,2743,2757$, $2764,2769,2775,2778,2781,2784,2788$, $2804,2813,2821,2828,2839,2851,2868$, 2874, 2880, 2882, 2886. (Fig. 4.) 


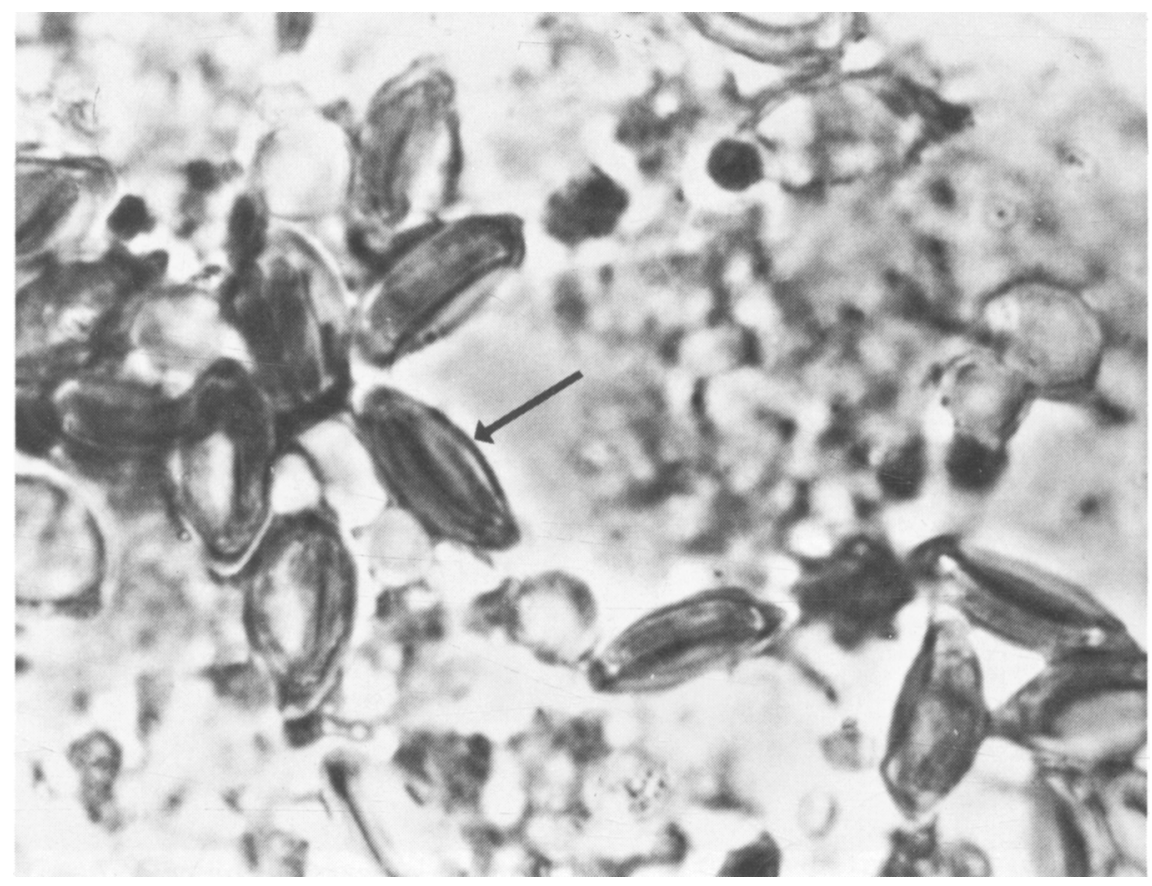

Fig. 4. Acc. No. 2524: Giemsa-stained fat-body smear from Tribolium castaneum infected with the schizogregarine, Farinocystis tribolii. Arrow shows mature spores. Magnification= $1200 \mathrm{X}$.

\section{Tribolium confusum Jacqueline duVal}

2042 et al.-A total of 59 accessions of specimens of the confused flour beetle were submitted by the Department of Genetics, University of California, Berkeley, California, during the period April 15, 1963, to November 28, 1967. These specimens were either from stock or experimental cultures and were submitted for routine pathogenic examination. Diagnoses: Of the 59 accessions, 42 were non-microbial; 14 showed a microsporidiosis caused by Nosema whitei; one showed a coccidiosis caused by Adelina tribolii and two showed a bacteriosis caused by Streptococcus faecalis. Accession numbers included: 2042, 2193, 2202, 2209, 2220, 2247, 2263, 2280B, 2333, 2356, 2393, 2483, 2490, 2495, 2499, 2507, 2508, 2511, 2517, 2520, 2523, 2529, 2537, 2542, 2544, 2549, 2551, $2559,2570,2572,2576,2581,2584,2587$, $2602,2608,2617,2622,2630,2633,2645$,
$2647,2662,2669,2672,2674,2678,2698$, $2701,2702,2705,2707,2710,2712,2725$, 2731, 2742, 2747, 2789. (Fig. 5.)

\section{Tribolium destructor Uyttenboogaart}

2372, 2472, 2484, 2797, 2553, 2562, 2663 -Seven accessions of this flour beetle were submitted by the Department of Genetics, University of California, Berkeley, California, from March 24, 1965, to August 2, 1966, and were from the department's stock or experimental cultures. Routine examination for pathogens was requested. Diagnoses: Four showed a bacteriosis caused by Streptococcus faecalis; one showed a microsporidiosis by Nosema whitei and two were non-microbial.

\section{Tribolium madens Charp}

2428, 2431, 2464, 2481, 2502, 2509, 2512, 2521, 2531-These nine accessions were received from the Department of 


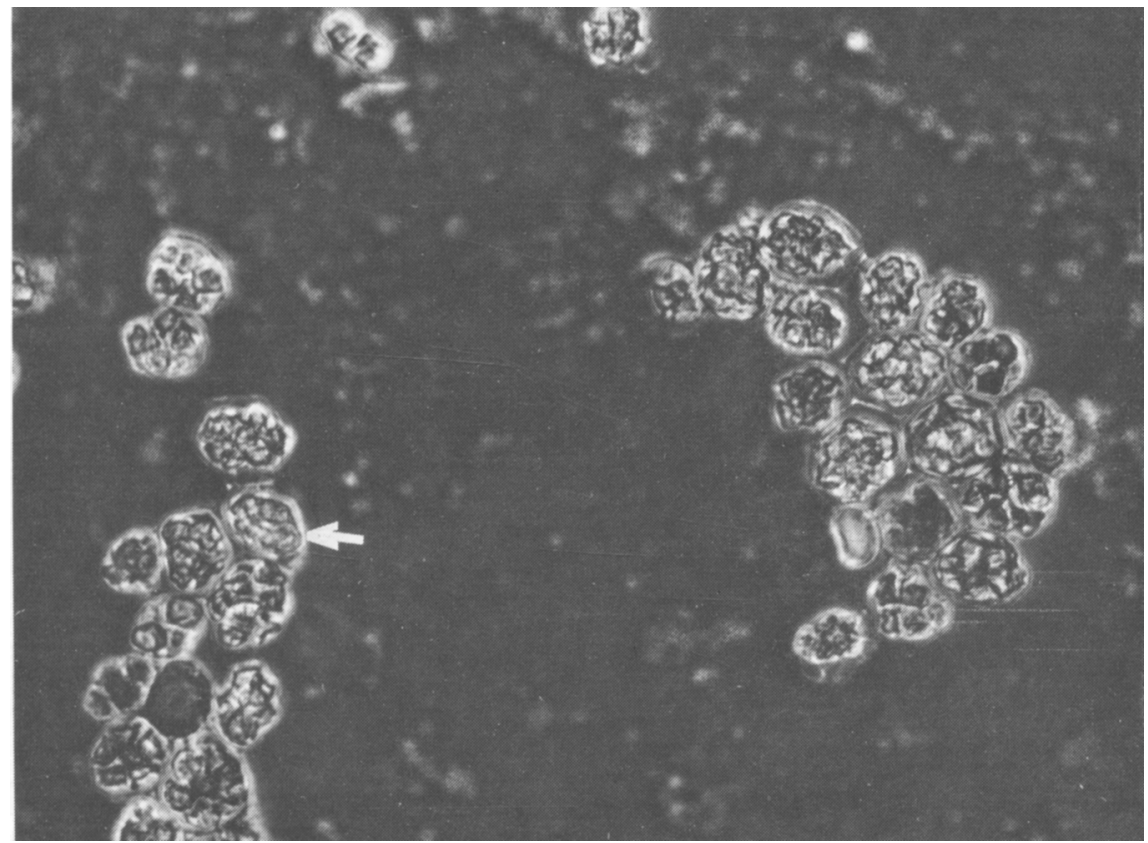

Fig. 5. Acc. No. 2280B: Phase-contrast micrograph of a giemsa-stained fat-body smear from Tribolium confusum infected with the coccidian Adelina tribolii. Arrow shows immature oöcyst. Magnification $=800 \mathrm{X}$.

Genetics, University of California, Berkeley, California, from July 6, 1965, to December 5,1965 , with the request for routine pathogenic examinations. The submitted material came either from stock or experimental cultures. Diagnoses: Five accessions showed a bacteriosis caused by Streptococcus faecalis; one showed a microsporidiosis caused by Nosema whitei; and three were non-microbial.

\section{Tribolium sp.}

2069-Live larvae and pupae were submitted from a laboratory stock culture by J. V. Slater, Berkeley, California, on June 12, 1963. Submitted larvae appeared to be healthy. Some of the pupae exhibited local discoloration, but otherwise appeared normal. A thorough examination of the submitted specimens showed no microbial etiology. Diagnosis: Nonmicrobial.

\section{Unidentified}

3002-One dead adult beetle preserved in alcohol was submitted by $\mathrm{L}$. Magaw, Quincy, California, on July 26, 1971. Diagnosis: Nematomorphosis caused by a Gordius sp.

\section{COLLEMBOLA}

\section{Entomobryidae}

\section{Entomobrya unostrigata}

2822-Several dead nymphs from a laboratory culture were submitted by J. E. Lauck, Davis, California, on April 25,1968 . The cultures were contaminated with various fungi, and their identification was requested to determine if any were known insect pathogens. Diagnosis: Saprophytic fungi present included Tritirachium sp., Aspergillus niger, and an unidentified Dematiaceae.

2826 - Three cultures were submitted by J. Lauck on May 8, 1968 for the 
identification of fungal contaminants. Four fungi were isolated, one of which was an insect pathogen. Diagnosis: Saprophytic fungi included a yeast, an unidentified Dematiaceae, and Rhizopus nigricans. The pathogenic fungus was Paecilomyces farinosus (=Spicaria farinosa). This isolate was tested for pathogenicity against Galleria melonella $\mathrm{L}$. with positive results.

\section{DERMAPTERA}

\section{Carcinophoridae}

\section{Euborellia cinticollis (G.erstaeker)}

2729-Two adult specimens were received from J. Knabke, Davis, California, on February 21, 1967. Diagnosis: Probably non-microbial, or if microbial, primary etiology had been obscured by secondary fungi and bacteria.

2897-A few adult earwigs from a laboratory culture were submitted by J. Knabke, Davis, California, on June 25, 1969. Diagnosis: Mycosis caused by an Entomophthora sp.

\section{Forficulidae}

\section{Forficula auricularia (L.)}

2082-These specimens were collected in Linden, California, by L. Falcon, on July 11, 1963. Diagnosis: No pathogens found.

2086-Several specimens of this earwig were submitted by R. Schoeppner, Berkeley, California, on July 19, 1963. Diagnosis: Mycosis caused by Entomophthora sp. near $E$. forficulae.

2560-One dead specimen was submitted by J. Powell, Berkeley, California, on January 21, 1966. Diagnosis: Mycosis: Beauveria bassiana.

2008-Several laboratory reared specimens were submitted by $R$. Schoeppner, Berkeley, California, on February 5, 1963. Diagnosis: Secondary mycoses caused by Fusarium sp. and Mucor sp., and a slime mold near Stemonitis.

\section{DIPTERA}

Agromyzidae

Ophiomyia sp.

2155-One adult agromyzid was submitted by C. E. Stegmaier, Hialeah, Florida, on September 9, 1963. Diagnosis: Mycosis caused by a member of the Aspergillus ochraceus group.

\section{Unidentified specimen}

2187-One specimen was submitted by S. Prasertphon, Berkeley, California, on October 17, 1963. Diagnosis: Mycosis caused by Entomophthora sphaerosperma.

\section{Anthomyiidae}

Hylemya spp. platura (Meig.) \& florilega Zett.

2791-Three, dry, pinned, adults were received from $\mathrm{A}$. G. Wheeler, Ithaca, New York, on December 18, 1967. Diagnosis: Mycosis caused by Entomophthora muscae.

\section{Ophyra leucostoma Wied.}

2279-One adult from Sonoma County, California, was submitted by J. Poorbaugh, Berkeley, California, on June 18, 1963. Diagnosis: Mycosis caused by Entomophthora sp., probably E. muscae.

\section{Blepharoceridae}

\section{Neocurupira campbelli Dumbleton}

2063-Several specimens were submitted by G. A. H. Helson, Wellington, New Zealand, on June 5, 1963. Diagnosis: Mycosis caused by an aquatic Phycomycete.

2235-Four vials of preserved specimens submitted by G. A. H. Helson, Wellington, New Zealand, had been collected from January 1963 to February 1964. Diagnosis: Vial 1. Mucoraceous fungus, probably Mucor, or possibly a new species and genus. Vials 2 and 3 . Mites belonging to the Trombidioidea: 


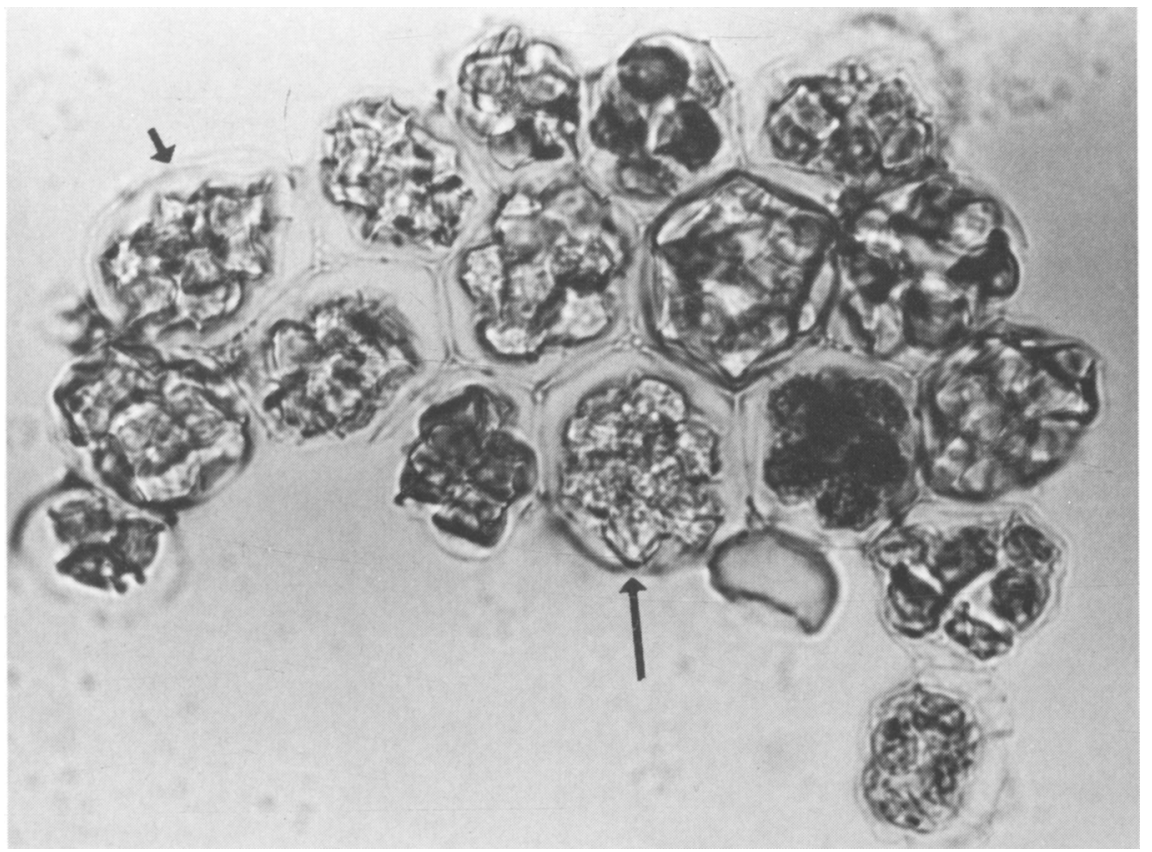

Fig. 6. Acc. No. 2306: Immature oöcysts (arrows) of an Adelina sp. in an alcohol-fixed giemsa-stained smear from Chaoborus astictopus. Magnification $=1000 \mathrm{X}$.

Johnstonianidae, apparently an undescribed genus intermediate between the subfamilies Johnstonianinae and Lasseniinae. Vial 4. Saprolegnia sp.-saprophytic.

\section{Cecidomyidae}

\section{Haplodiplosis equestris (B. Wagner)}

2896-Several adults of the saddle gall midge from barley were submitted on June 24, 1969 by Th. Thygesen, Lyngby, Denmark. Diagnosis: Nonmicrobial.

\section{Chaoboridae}

Chaoborus astictopus Dyar and Shannon

2306-Several specimens and histological sections were submitted by $P$. Sikorowski, Lakeport, California, July 31,1964 . The larvae were collected at Pinto Lake, Santa Cruz County, California, and appeared granular and grayish in color, whereas normal larvae are colorless. In Pinto Lake, about 1 per cent of the 4th instar larvae were infected. Diagnosis: Protozoan disease caused by a Coccidian, Adelina sp. New Record. (Fig. 6.)

2330 - Cultures of a bacterium isolated from the Clear Lake gnat were submitted by P. P. Sikorowski, Lakeport, California, on October 16, 1964. These cultures were thought to be a gram positive spore former, but examination showed them to be gram negative, non-spore forming. Requests for additional cultures were not fulfilled. Diagnosis: Aerobacter sp.

2700-One bacterial culture isolated from the Clear Lake gnat was received from P. P. Sikorowski, Lakeport California, on October 15, 1966. Diagnosis: Pseudomonas aeruginosa.

2774-Several larvae of the Clear Lake gnat were received from P. Sikorowski, Lakeport, California, on Sep- 
tember 20, 1967. Confirmation of a possible coccidian infection was requested. Diagnosis: Coccidiosis caused by an Adelina sp. (Fig. 7.)

\section{Chironomidae}

Chironomus plumosus (L.)

2210 - Two slides of a microsporidian isolated from this chironomid were submitted by $\mathrm{O}$. L. Lovett, Madison, Wisconsin, on December 12, 1963. Diagnosis: Thelohania n. sp. related to $T$. pinguis. New record-new pathogen.

\section{Chloropidae}

\section{Thaumatomyia glabra (Meigen)}

2859-On September 24, 1968, one adult from an alfalfa field was received from A. G. Wheeler, Ithaca, New York. Diagnosis: Mycosis caused by Entomophthora muscae.
Cordyluridae

Scopeuma stercorarium (L.)

2254 -One adult was submitted by J. Poorbaugh, Berkeley, California, on April 17, 1964. Diagnosis: Non-microbial.

\section{Culicidae}

\section{Aedes aegypti (Linn.)}

2973-On November 16, 1970, C. L. Judson, Davis, California, sent several dead mosquito larvae from his laboratory culture. Two strains of gram negative bacteria were isolated and tested against mosquitoes in our laboratory, with negative results. Diagnosis: Probably non-microbial, or if a primary microbial etiology was present, it has been masked by the action of secondary bacteria.

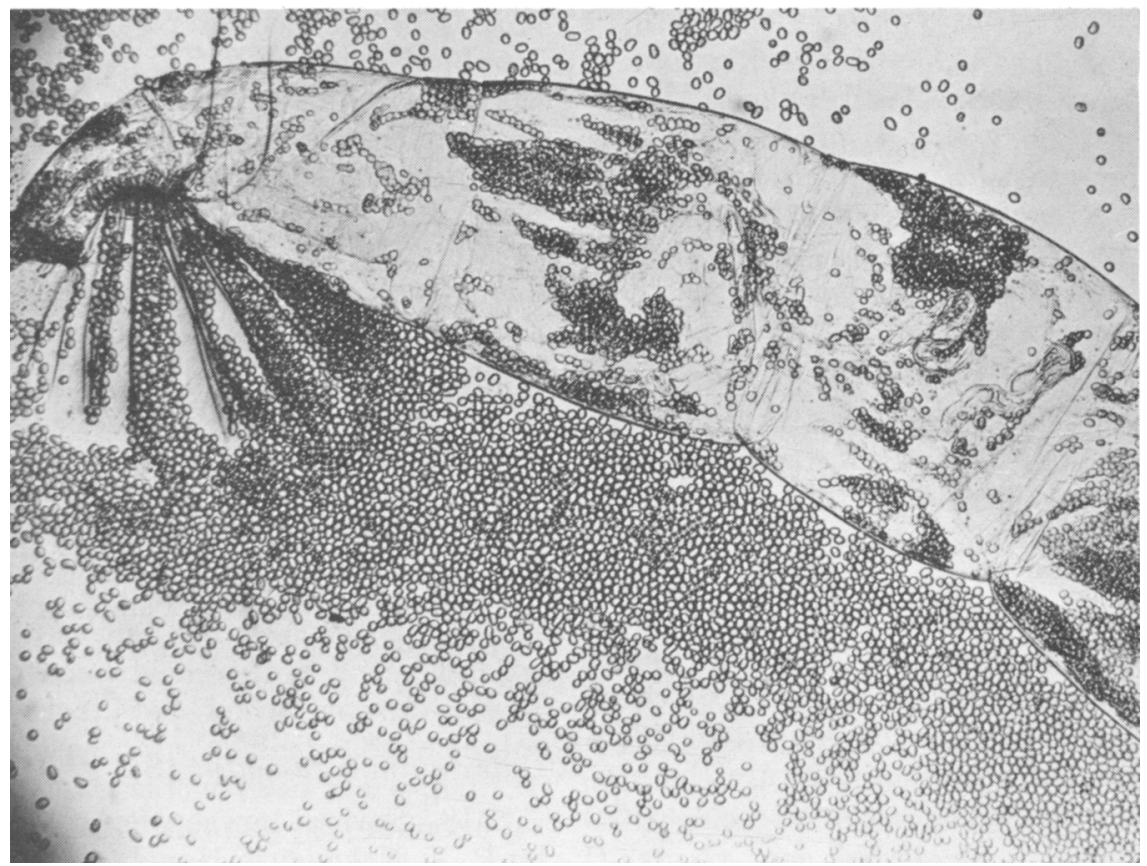

Fig. 7. Acc. No. 2774: Chaoborus astictopus larva infected with Adelina sp. The larval cuticle ruptured, releasing oöcysts which had completely filled the host's body cavity. Oöcysts appear empty at this low magnification due to the high refractivity of their thick walls. Magnification= $40 \mathrm{X}$. 


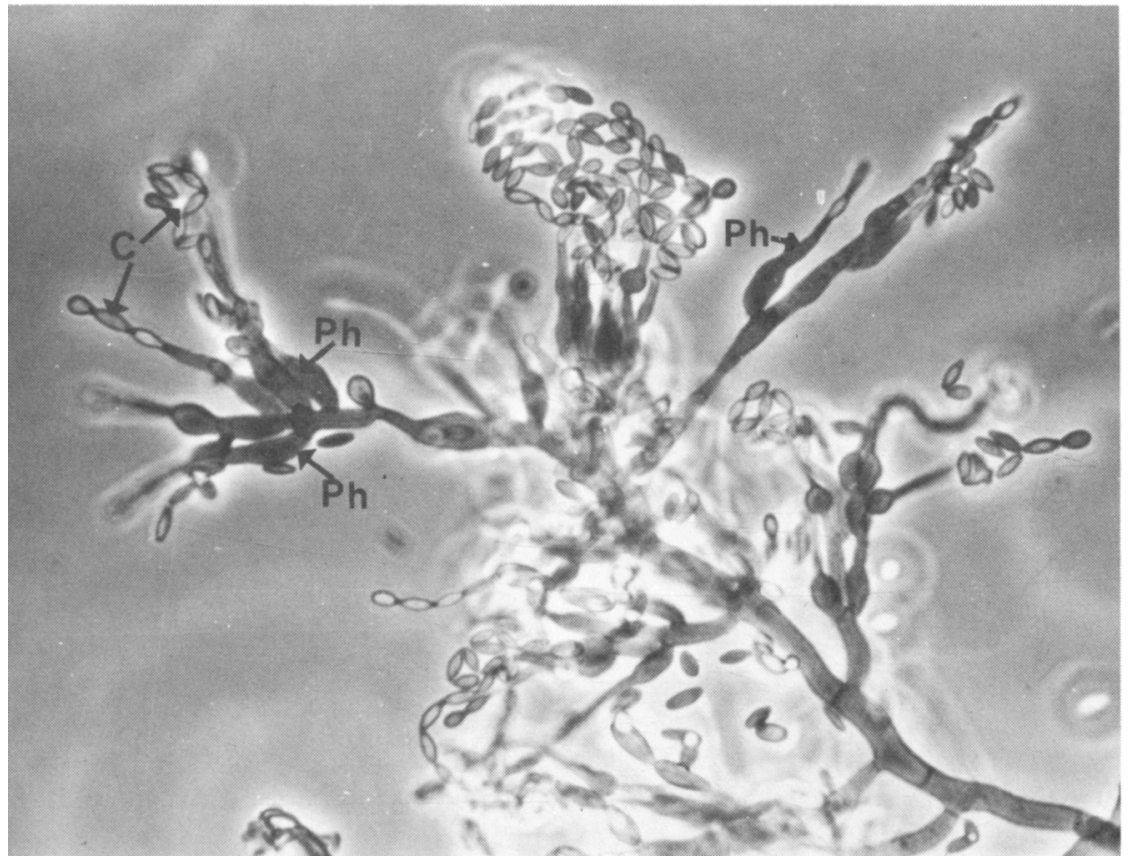

Fig. 8. Acc. No. 2720: A sporulating culture of Paecilomyces fumoso roseus isolated from Aedes sollicitans. Phialides (Ph) ; Conidia (C). Magnification $=800 \mathrm{X}$.

\section{Aedes sollicitans (Walker)}

2720-A fungus culture from this mosquito was submitted by T. Clark, Fresno, California, on January 12, 1967. Diagnosis: Mycosis: Paecilomyces fumoso-roseus. (Fig. 8.)

\section{Aedes togoi (Theobald)}

2025-Ten laboratory-reared larvae were submitted by B. R. Laurence, London, on March 12, 1963. Diagnosis: Undetermined.

\section{Aedes vexans (Mg.)}

2463-A bacterium isolated from larvae was submitted by W. R. Kellen, Fresno, California, on August 24, 1965. It was not possible to culture the bacterium, so identification was not made.

\section{Culex pipiens fatigans Wied.}

2966-Several laboratory-reared larvae preserved in alcohol were submitted on August 29, 1970 by H. N. Espinola,
Belo Horizonte, Brazil. The specimens had a black plug near the trachea in the thorax. Dissection revealed the plug to be fungal in nature. However, preservation and the absence of reproductive structures prevented identification. Diagnosis: Possible mycosis.

2980-Several additional larvae were received from Dr. H. N. Espinola on January 28, 1971. These were shipped in water and were rather deteriorated upon arrival. Thus, the fungus causing the trachael plugs mentioned above was not viable, and no identification could be made. Diagnosis: Possible mycosis.

\section{Culex quinquefasciatus (Say)}

2418-Several larvae were submitted by P. Y. Nakagawa, Honolulu, Hawaii, on June 14, 1965. They had been collected in an area which had been exposed to insecticidal treatment with EC, DDT and Baytex (Fenthion) granules 
as larvacides. Diagnosis: Bacterioses caused by Bacillus cereus var. mycoides, and Pseudomonas aeruginosa.

\section{Culex tarsalis Coquillett}

2965-One frozen adult was submitted by G. Grodhaus, Berkeley, California, on September 11, 1970. The specimen had been collected by $\mathrm{K}$. $\mathrm{H}$. Hansgen; Lincoln, Placer County, California, on August 31, 1970, and showed a yellow sphere on one eye. It was a pollen grain which had become stuck to the eye with a bit of nectar. Diagnosis: Non-microbial.

\section{Culicida sp.}

2841-Several laboratory reared larvae were submitted on June 24, 1968 by L. Ehler, Berkeley, California. Diagnosis: Non-microbial.

\section{Unidentified mosquito larva}

2396-One larva was found in a roadside ditch by B. T. Whitworth, Willows, California, on April 27, 1965. Diagnosis: Bacterioses caused by Clostridium sp. and Pseudomonas aeruginosa.

\section{Drosophilidae}

\section{Drosophila melanogaster Meigen}

2697-Several adult flies were submitted by F. K. Ho, Berkeley, California, on October 6, 1966. Diagnosis: Nonmicrobial.

2929 - Eggs, larvae, pupae and adults were submitted, on December 9, 1969, by F. H. Sobels, Leiden, the Netherlands. The material was from laboratory cultures in which egg hatch was slightly sub-normal, but larval mortality was high. Pupae were misshapen, and adults from these seldom lived more than 24 hours. Microbial analysis produced no known pathogenic micro-organisms, and electron micrographs showed no virus. However, the electron microscope examinations did show the salivary glands to be filled with yeast cells. Two yeasts were isolated and identified as Pichia membranaefaciens, and Candida sorbosa. It was not determined whether or not these might be pathogenic. Diagnosis: Inconclusive.

3032-Adult fruit flies, which were being used in investigations on quantitative intake of food under the influence of carbon dioxide, were submitted by S. S. Chawla, Quebec, Canada, on November 22, 1972. Diagnosis: Possible infection with sigma virus (from description of submitter), or non-microbial.

3037-Additional fruit fly adults from the above laboratory were received from S. S. Chawla, Quebec, Canada, on December 14, 1971. Diagnosis: Possible infection by sigma virus, or non-microbial.

\section{Glossinidae}

Glossina morsitans Westw.

2941-Adults and pupae of this tsetse fly were submitted by J. Fraga de Azevedo, Lisbon, Portugal, on March 19, 1970. The specimens were from laboratory colonies exhibiting a great number of abortions, reduction of longevity, reduction in size of pupae, and general weakness. However, thorough examination and microbial analysis showed no microbial etiology. Diagnosis: Nonmicrobial.

\section{Muscidae}

\section{Fannia canicularis (L.)}

1886-One adult was submitted by R. D. Frye, Fargo, North Dakota, on June 1, 1962. Diagnosis: Mycosis caused by Entomophthora muscae.

1980_One adult specimen was submitted by J. H. Poorbaugh, Berkeley, California, on November 15, 1962. Diagnosis: non-microbial.

\section{Haematobia irritans (L.)}

2843-Larvae from laboratory cultures were submitted by H. G. Kinzer, 
University Park, New Mexico, on June 26, 1968. Diagnosis: Bacterioses caused by Bacillus cereus and $B$. cereus var. mycoides.

\section{Hylemyia antiqua (Meigan)}

1925-Several adults and pupae were submitted by J. Ellington, Ithaca, New York, on August 9, 1962. They had been laboratory reared. Diagnosis: Undetermined.

\section{Hylemyia seneciella (Meade)}

2079_Three adults collected in Paris, France, were submitted by L. A. Andres, on July 5, 1963. Diagnosis: Mycosis caused by Entomophthora muscae.

\section{Musca domestica (L.)}

1836 - A single adult was submitted by H. Scott, Jr., Berkeley, California, on January 22, 1962. Diagnosis: Mycosis caused by Entomophthora muscae.

1979_Eight adults were submitted by J. H. Poorbaugh, Berkeley, California, on November 15, 1962. Diagnosis: Mycosis caused by Entomophthora muscae.

2457-Several laboratory-reared larvae were submitted by J. Poorbaugh, Berkeley, California, on August 16, 1965. Diagnosis: Mycosis caused by Aspergillus flavus and bacteriosis caused by Bacillus cereus.

\section{Stomoxys calcitrans (L.)}

1981-A single adult was submitted by J. H. Poorbaugh, Berkeley, California, on November 15, 1962. Diagnosis: Non-microbial.

Unidentified (anthomyiid-Hylemya and Pegomyia group)

1903-Three adults were submitted by R. D. Frye, Fargo, North Dakota, on July 20, 1962. Diagnosis: Mycosis caused by Entomophthora muscae.

\section{Unidentified Anthomyiid}

2016-One adult was submitted by J. Linsley Gressitt, Honolulu, Hawaii, on February 19, 1963. Diagnosis: Nonmicrobial.

2032-Nine dead adults were submitted by D. M. Peterson, San Pedro, California, on March 25, 1963. Diagnosis: Mycosis caused by Entomophthora muscae.

\section{Unidentified Muscoid fly}

2267-Eight adults collected in Bowman, California, were submitted by $R$. W. Gaetjen, Berkeley, California, on June 4, 1964. Diagnosis: Mycosis caused by Entomophthora sp.

\section{Otitidae}

Tetanops myopaeformis (Roder)

2527-Eight dead dry specimens were submitted by $K$. Shea, Twin Falls, Idaho, on November 29, 1965. Collected in Ruper, Idaho. Diagnosis: Mycosis: Metarrhizium anisopliae.

\section{Sciaridae \\ Unidentified}

2165-Many larval specimens were submitted by W. Steffan, Berkeley, California, on September 16, 1963. They had been reared on artificial media. Diagnosis: Non-microbial.

2188-A single adult female was submitted by W. Steffan, Berkeley, California, on October 18, 1963. Diagnosis: Mycosis caused by Entomophthora sp. near $\boldsymbol{E}$. dipterigena.

2462-One laboratory-reared adult was submitted by W. Brooks, Berkeley, California, on August 24, 1965. Diagnosis: Mycosis caused by Entomophthora $\mathrm{sp}$.

2660-Several adults were received from G. O. Poinar, Jr., Berkeley, California, on August 2, 1966. Diagnosis: Mycosis: Entomophthora sp. 


\section{Simuliidae}

Unidentified

2058-Fourteen larvae collected near Danville, California, were submitted by J. R. Anderson, Berkeley, California, on May 27, 1963. Diagnosis: Microsporidiosis caused by Thelohania sp., probably $T$. fibrata.

\section{Syrphidae}

Melanostoma.sp.

2650-One larva was submitted by M. A. Mattar, La Cruz, Chile, on July 13, 1966. Diagnosis: Bacterioses, secondary: Proteus sp. and Alcaligenes sp.

\section{Syrphus americana (Wiedeman)}

1876_-Seventeen adults were submitted by G. M. Buxton, Sacramento, California, on May 17, 1962. Diagnosis: Mycosis caused by Entomophthora muscae.

Syrphus octomaculatus Walker 2675-Two larvae were received from M. A. Mattar, La Cruz, Chile, on August 18, 1966. Diagnosis: Bacteriosis: Non-chromogenic strain of Serratia marcescens.

\section{Syrphus torvus O. Sacken}

2281-These larvae were submitted on June 26, 1964 by G. D. Amman, Asheville, North Carolina. They were part of a laboratory colony and were completely deteriorated. A bacterium that has been known to cause epizootics in the laboratory was isolated. Diagnosis: Possible bacteriosis: Pseudomonas aeruginosa.

\section{Syrphus sp.}

2649-Two shriveled larvae were submitted by M. A. Mattar, La Cruz, Chile, on July 13, 1966. Examination showed an abundance of bacteria. Analysis provided cultures of Proteus sp. and Alcaligenes sp., both secondary invaders. Diagnosis: Either non-micro- bial, or the primary etiology has been obscured by the action of a secondary bacterial infection.

\section{Unidentified}

2759-Several adults were submitted by T. Gordon, Oakland, California, on July 10, 1967. Diagnosis: Mycosis: Entomophthora muscae.

2793-One pinned adult specimen was submitted by A. G. Wheeler, Ithaca, New York, on Deeember 18, 1967. Diagnosis: Mycosis caused by Entomophthora sphaerosperma.

\section{Psychodidae}

Phlebotomus sanguinarius (Fairchild and Hertig)

1874 - A single adult was submitted from a laboratory culture by P. T. Johnson, Balboa Heights, Canal Zone, on May 15, 1962. Diagnosis: Undetermined.

Phlebotomus vexator occidentis (Maugabeicot and Galiudo)

2336-Some specimens were submitted from a laboratory culture by B. Chaniotis, Berkeley, California, on November 2, 1964. A bacteriosis was discovered which was probably secondary. Diagnosis: Bacteriosis caused by Pseudomonas sp.

\section{Tabanidae}

Chrysops vittatus Wied.

2765-One pupa was received from P. H. Thompson, New Brunswick, New Jersey, on July 31, 1967. Diagnosis: Probable bacteriosis, caused by Bacillus cereus, with secondary invasion by an Aerobacter sp.

\section{Tabanus sp.}

2766-One larva was submitted by P. H. Thompson, New Brunswick, New Jersey, on July 31, 1967. Diagnosis: Proteus morganii and Alcaligenes sp. isolated as secondary saprophytic bac- 
teria. Primary cause of death may not have been microbial, or may have been obscured by the action of secondary saprophytes.

2771-Three larvae were submitted by P. H. Thompson, New Brunswick, New Jersey, on August 22, 1967. Diagnosis: Bacteriosis: Pseudomonas aeruginosa.

\section{Tachinidae}

Lespesia sp.

2442-Two endoparasites of the banana bagworm were collected and submitted by F. Lara, Limon, Costa Rica, on July 28, 1965 . The cadavers were filled with mites and the primary etiology was obscured. Diagnosis: Undetermined.

\section{Lixophaga diatraeae (Townsend)}

1939 - Several laboratory-reared adults were submitted by L. W. van Whervin, Barbados, West Indies, on September 6, 1962. Diagnosis: Mycosis caused by Entomophthora sp.

1998-Several laboratory-reared specimens were submitted by L. W. van Whervin, Barbados, West Indies, on December 27, 1962. Diagnosis: Mycosis caused by Entomophthora sp. near apiculata.

\section{Tephritidae}

\section{Ceratitus capitata (Wiedeman)}

2736-Many adults were submitted by E. Naton, Munich, West Germany. The specimens were collected on March 25, 1967 and submitted on April 3, 1967. Diagnosis: Probably non-microbial.

\section{Dacus dorsalis (Hendel)}

2406_-Several adults were submitted by R. T. Cunningham, Hilo, Hawaii, on May 13, 1965. Examination showed only secondary saprophytic fungi and bacteria. Diagnosis: Probably non-microbial.

\section{Dacus oleae (Gmelin)}

1854-Many pupae were submitted by T. C. Buchelos, Patras, Greece, on March 15, 1962. Diagnosis: No pathogen observed.

\section{Rhagoletis completa (Cresson)}

2179_Seven dead larvae were submitted by L. A. Falcon, Berkeley, California, on October 7, 1963. They had been collected in Linden, California, in an area which had been treated with Bacillus thuringiensis. Diagnosis: Nonmicrobial.

2515-Several dead pupae submitted by J. Shimizu, Albany, California, were collected in Manteca, California, from April to July, 1965. Diagnosis: Probable Aspergillosis: Aspergillus flavus. In addition, a Fusarium was also isolated and a bacterium, Aerobacter, sp. It would appear that Aspergillus was the microbial etiological agent.

2922-An agar plate containing bacteria isolated from the walnut husk fly was submitted on October 17, 1969 by K. Hagen, Albany, California, with a request for identification. Diagnosis: The bacteria were identified as Aerobacter sp., and Aerobacter cloacae.

\section{Tipulidae}

\section{Tipula graminivora (Alexander)}

1855-Several specimens were collected near Petaluma, California, and submitted by W. H. Lange, Davis, California, on March 15, 1962. Specimens showed signs of a mycosis. Diagnosis: Possible mycoses caused by Fusarium sp., Mucor sp. and Fumago sp.

\section{Tipula quaylii (Doane)}

2233-Three specimens were collected at Herald, California, by $\mathbf{H}$. Lange, Davis, California, on February 19, 1964. Diagnosis: Undetermined. 


\section{Tipula sp.}

2218-Nine larvae were submitted by W. H. Lange, Davis, California, on January 3, 1964. The disease appeared to be an epizootic. Diagnosis: Undetermined.

2590-A few dead specimens collected in Petaluma, California, on March 26, 1966, were submitted by W. H. Lange, Davis, California. Diagnosis: General bacterioses: Bacillus cereus, Streptococcus faecalis and Pseudomonas aeruginosa.

\section{Trypetidae}

\section{Anastrepha suspensa (Loew)}

2780-Several adults of the Caribbean fruit fly were received from $R$. B. Marlatt, Homestead, Florida, on October 11, 1967. Diagnosis: Mycosis caused by Entomophthora echinospora.

2794-Several brown and shriveled larvae were submitted by R. B. Marlatt, Homestead, Florida, on December 26, 1967. Diagnosis: Non-microbial.

\section{Xylophagidae}

Inopus rubiceps Macquart (=Altermetoponia rubiceps [Macquart] Stratiomyida)

2500_-Some specimens were collected in Colma, California, by L. Campbell, Berkeley, California, on October 22, 1965. Diagnosis: External saprophytic fungi and general bacterial deterioration.

\section{Unidentified}

2028-One pupa was submitted by G. A. H. Helson, Wellington, New Zealand, on March 18, 1963. Diagnosis: Mycosis caused by Beauveria bassiana.

2547-Many dead specimens preserved in alcohol were submitted by $\mathrm{S}$. Shima, Jr., Palmira, Valle, Colombia, South America, on December 9, 1965. They were collected in La Torre, Palmira, Valle. Diagnosis: Non-microbial; examination of whole and dissected specimens revealed no microbial etiology.

2556-Many dead specimens submitted by S. Shima, Jr., Palmira, Valle, Colombia, South America, on January 10, 1966. They were collected in December, 1965, in La Torre, Palmira, Valle. Diagnosis: Mycosis: Spicaria rileyi.

2815-Fungus cultures isolated from a cane fly were submitted by B. M. Hogg, Mandeville, Jamaica, West Indies, on March 28, 1968. Diagnosis: The fungus, Metarrhizium anisopliae, was present.

\section{HEMIP'TERA (HETEROPTERA)}

\section{Anthocoridae}

\section{Anthocoris sp.}

2375-One dead adult was submitted by J. Shimizu, Berkeley, California, on March 29, 1965. Diagnosis: Non-microbial. A secondary fungus, Penicillium sp., which was external only, had not colonized the insect.

\section{Cimicidae}

\section{Paracimex n. sp.}

1887-N. Ueshima, Berkeley, California submitted eight bedbugs on June 7, 1962. The bedbugs had been sent to him from Kuala Lumpur, Malaya, where they had been collected from the nests of Swifts. Diagnosis: Mycosis, caused by Aspergillus flavus. A new host record for this fungus. (Fig. 9.)

\section{Lygaeidae}

Ligyocoris n. sp. (nr. L. sobrius (Uhler))

1984 - Two adults were submitted by J. A. Chemsak, Berkeley, California, on November 30, 1962. The specimens had been collected by P. D. Ashlock; Carson Ridge near Woodacre, Marin County, California, from under the bark of a dead Sargent's Cypress. Di- 


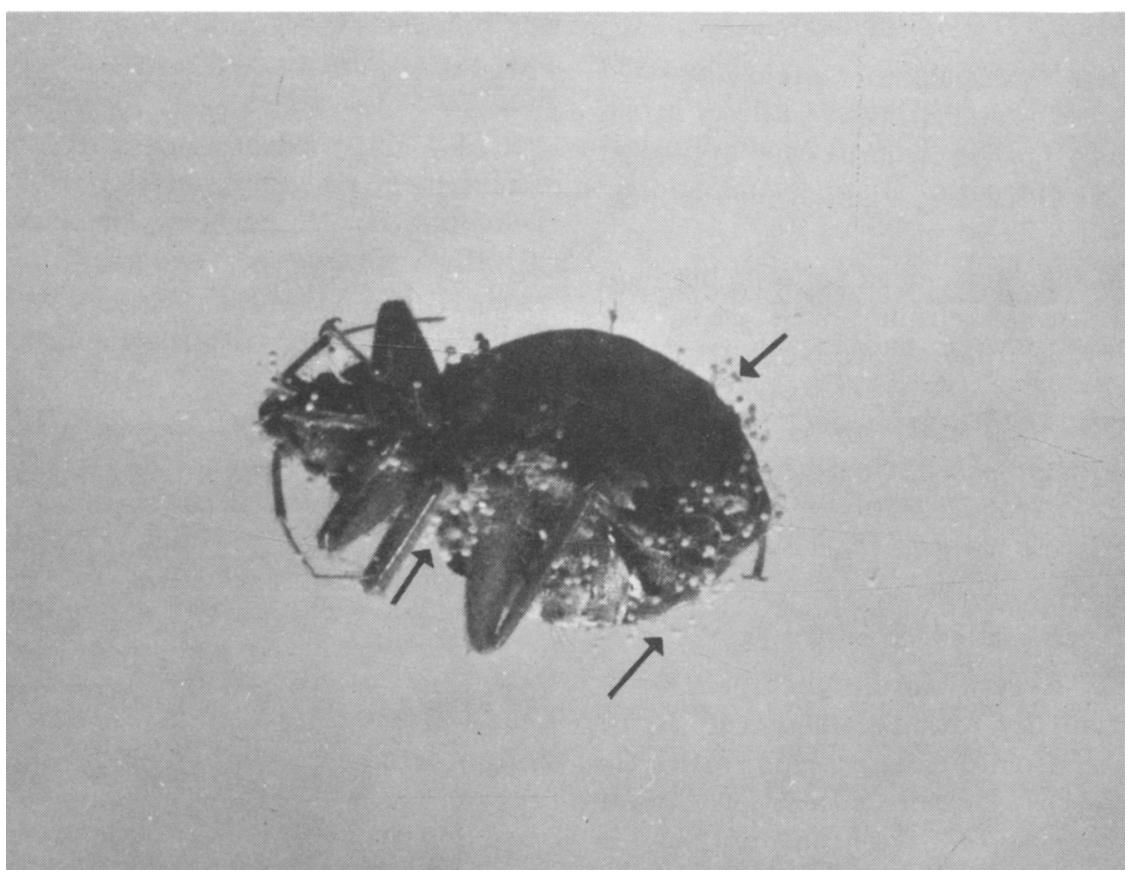

Fig. 9. Acc. No. 1887: Paracimex sp. infected with Aspergillus flavus. Note sporophores of fungus protruding from intersegmental folds (arrows). Magnification $=12 \mathrm{X}$.

agnosis: Mycosis caused by Beauveria bassiana. A new host record for this fungus.

\section{Miridae}

Adelphocoris lineolatus (Goeze)

2855-One adult and three nymphs collected from alfalfa at Ithaca, New York, were submitted by A. G. Wheeler, Jr., on August 20, 1968. Diagnosis: Mycosis caused by Entomophthora erupta.

\section{Helopeltis clavifer Walker}

1931-One specimen was submitted by A. Catley, Papua, New Guinea, on August 17, 1962. This mirid is the most important pest of cacao in the territory. Diagnosis: Mycosis caused by Beauveria bassiana. Probably a new host record for this fungus.

Lygus lineolaris (Palisot de Beauvois) 2672-One preserved specimen was submitted by R. F. Ruppel, East Lan- sing, Michigan, on November 9, 1970. Diagnosis: Mycosis caused by an unidentified Hyphomycete.

\section{Unidentified Miridae}

2651-Several adults were received from J. Hamai, Albany, California, on July 14, 1966. Diagnosis: Bacteriosis: Bacillus cereus.

\section{Nabidae}

Nabis sp.

2593-Two dead specimens were submitted by J. Shimizu, Albany, California, April 6, 1966. Diagnosis: Bacterioses: Bacillus cereus and Serratia marcescens.

\section{Zelus sp.}

2699-Several nymphs of this predator were submitted by J. Shimizu, Berkeley, California, on October 23, 1966. The specimens had been reared in 
the laboratory on an artificial diet. Diagnosis: Non-microbial.

\section{Pentatomidae}

\section{Bathycoelia thalissima}

2341-J. T. Legg, Tafo, Ghana, submitted a fungus culture isolated from B. thalissima on November 18, 1964. Diagnosis: A non-entomogenous fungus, Cephalosporium sp.

\section{Dinocoris melanoleuca Westwood}

2908-Adults and nymphs of this pentatomid were submitted by L. A. Stange, San Miguel de Tucuman, Argentina, on August 21, 1969. Diagnosis: Mycosis caused by Metarrhizium brunneum.

\section{Podisus maculiventris (Say)}

2632-One nymph was received from J. Shimizu, Albany, California, on June 13, 1966. Diagnosis: Possible bacteriosis caused by a gram negative rod in either the Haffnia grp. or an Erwinia sp.

\section{Podisus serieventris Uhl.}

2597-One dead nymph was submitted by D. H. Dahlston, Berkeley, California, in August, 1965. The specimen was collected at Knox Mt., 30 miles southwest of Alturas, Modoc Co., California. Diagnosis: Bacteriosis caused by Bacillus thuringiensis var. thuringiensis.

\section{Pyrrhocoridae}

Dysdercus cardinalis Gerst.

3027--Eggs, nymphs, and adults of the cotton stainer collected in Nairobi, Kenya, and reared in Amsterdam, the Netherlands, were submitted by L. van der Geest on November 19, 1971. Diagnosis: Non-microbial.

\section{Dysdercus intermedius Distant}

2796-Five adult cotton stainers preserved in Bouin's fixative were received from J. L. Vincent, Devon, England, on
December 26, 1967. Diagnosis: Nonmicrobial.

\section{Reduviidae \\ Triatoma rubida (Uhler)}

2334 - Two specimens were submitted on October 23, 1964, by R. Watkins, San Francisco, California. The hemocoel was filled with small gram negative rods which failed to grow on various artificial media. On October 29, 1964, Mrs. Watkins brought in a culture of the bacterium on blood agar. This bacterium was identified as a Pseudomonas sp. Diagnosis: Bacteriosis, possibly caused by a Pseudomonas sp., but not confirmed by infectivity tests.

\section{Ricaniidae}

Scolypopa australis (Walker)

2273 - Some specimens were submitted from Tambourine Mountain, Queensland, Australia, by J. H. D. Barnett, Brisbane, Australia, on June 12, 1964. Diagnosis: Mycosis caused by Hirsutella citriformis.

\section{HEMIPTERA (HOMOPTERA)}

\section{Adelgidae \\ Adelges piceae (Ratz)}

2783-Adults and eggs of the balsam wooly aphid on bark dises of Abies fraseri were submitted by G. F. Fedde, Asheville, North Carolina, on November 3,1967 . The specimens were collected on October 25, 1967 in Mt. Mitchell State Park, North Carolina. Diagnosis: Mycosis caused by a Fusarium sp.

2790-Additional specimens of the balsam wooly aphid were received from G. F. Fedde, Asheville, North Carolina, on December 12, 1967. These specimens were again submitted on Abies fraseri bark sections. Diagnosis: Mycosis caused by a Fusarium sp. 


\section{Aleyrodidae}

Aleurodes barodensis Maskal

2229-Many nymphs of the sugar cane whitefly were submitted by $\mathrm{H}$. David, Madras State, India, on February 11, 1964. Diagnosis: Mycosis, caused by Aschersonia sp., in the Aleyrodisplacenta group.

\section{Bremesia tabaci (Gennadius)}

2971-Slides, and agar slant cultures of a fungus isolated from white flies in Chiangmai, Thailand, were received on November 10, 1970, from U. Sonpirode. Examination of the mounted specimens confirmed a mycosis and the fungus was identified as a Fusarium sp. Diagnosis: Mycosis caused by a Fusarium sp.

\section{Dialeurodes citrifolii (Morg.)}

2805-On February 8, 1968, several specimens of the cloudy winged whitefly were received from Q. L. Holdeman, La Ceiba, Honduras. Diagnosis: Mycosis caused by Aschersonia goldiana.

\section{Trialeurodes sp.}

1906-These specimens were submitted by J. L. Nickel from Cambodia on July 20, 1962. The insects were kept in a cyanide jar for several days. Diagnosis: The fungus, Cladosporium sp., was isolated from the dead specimens.

\section{Aphidae}

Acrothosiphon pisum (Harris)

2977-Many specimens were submitted by E. Zuniga-Salinas, La Cruz, Chile, on December 23, 1970. The material was field collected from alfalfa in Rancagua, Chile, where the disease had reached epizootic proportions in natural aphid populations. Diagnosis: Mycosis caused by Entomophthora aphidis.

\section{Aphis craccivora (Koch)}

2369-Several specimens were submitted by J. A. K. Farrell, Kilongwe, Malawi, Africa, on March 19, 1965. The disease is widespread in the field, and epizootic in the laboratory. Diagnosis: Mycosis caused by Entomophthora sp.

\section{Aphis gossypii (Glover)}

2302 - Several specimens of the melon aphid were submitted by $\mathrm{D}$. A. Wolfenbarger, Welasco, Texas, on July 22,1964 . They had been reared in a greenhouse. Diagnosis: Mycosis caused by Aspergillus sp. in the flavus-oryzae group.

Aphis helichrysi (Kaltenbach)

2265-Several specimens collected in Colma, California, were submitted by W. Allen, Berkeley, California, on June 1, 1964. Diagnosis: Mycosis caused by Entomophthora sp.

\section{Aphis sp. (possibly gossypii)}

1858-Several specimens were submitted by M. A. Janjua, Rawalpindi, Pakistan, on March 26, 1962. Diagnosis: No primary pathogen observed.

\section{Brevicoryne brassicae (L.)}

2741-Several adults were submitted by $\mathrm{D}$. White, Sydney, Australia, on April 14, 1967. Diagnosis: Bacteriosis: Pseudomonas aeruginosa.

Macrosiphum pechumani Mac Gillivray

2867-On October 18, 1968, four aphids collected from Convalaria majalis were submitted by A. G. Wheeler, Ithaca, New York. Diagnosis: Mycosis caused by Entomophthora aphidis.

\section{Macrosiphum pisi (Harris)}

1956-Several specimens were submitted by R. D. Frye, Fargo, North Dakota, on September 25, 1962. Diagnosis: Non-microbial.

\section{Metopolophium dirhodum (Wlk.)}

2873-Several rose-grass aphids were received on December 6, 1968 from $E$. Zuniga-Salinas, La Cruz, Chile, with 
reports of from 50 per cent to 75 per cent mortality in field populations. Diagnosis: Mycosis, caused by Entomophthora aphidis.

2877-On January 21, 1969, additional rose-grass aphids were received from E. Zuniga-Salinas, La Cruz, Chile. These specimens had been collected from barley and wheat near La Cruz. Diagnosis: Mycosis, caused by Entomophthora aphidis.

\section{Myzus persicae (Sulzer)}

1857-Several specimens were submitted by D. Purcifull, Davis, California, on March 23, 1962. Diagnosis: Mycosis caused by Entomophthora aphidis.

1862-_Some specimens were collected along the lower California coast and submitted by R. J. Shepard, Davis, California, on April 16, 1962. Diagnosis: Mycosis caused by Entomophthora aphidis.

1871-Some nymphs of the green peach aphid were collected from spinach near Painter, Virginia, by $R$. N. Hofmaster, and submitted by S. R. Dutky, Beltsville, Maryland, on May 5, 1962. Diagnosis: Mycosis caused by Entomophthora aphidis.

2274-Some specimens were collected at Redland Bay, Queensland, Australia, by J. H. D. Barnett, Brisbane, on June 12, 1964. Diagnosis: Mycosis caused by Entomophthora aphidis.

\section{Rhopalosiphum maidia (Fitch)}

2613 - A slide of a fungus isolated from this insect was submitted by R. B. Subba Rao, New Delhi, India, on May 10, 1966. Identification: Entomophthora sp.

1945-Some specimens were submitted by E. A. Wood, Stillwater, Oklahoma, on September 17, 1962. Diagnosis: Some were parasitized while the cause of death in others was unknown.

\section{Therioaphis trifolii (Monell)}

2475-Several slides of a fungus collected from this insect were submitted for identification by R. B. Subba Rao, New Delhi, India, on September 20, 1965. Identification: Entomophthora sp. probably $E$. aphidis.

\section{Unidentified root aphid}

1843-Several specimens were submitted by T. C. Lawrence, Berkeley, California, on February 23, 1962. Diagnosis: No pathogen found. Slight growth of Mucor sp.

\section{Unidentified}

1850 - A single nymph was submitted by D. Gerling, Berkeley, California, on March 13, 1962. Diagnosis: Mycosis caused by Entomophthora sp.

\section{Cercopidae}

Aeneolamia postica Urich \& Prosapia bicincta Say

3033-A mixture of adults and nymphs of these two spittlebugs collected in Aldama, Tamaulipas, Mexico, was submitted by J. Carrillo, Chapingo, Mexico, on November 23, 1971. Diagnosis: Mycosis caused by an Entomophthora sp.

\section{Philaenus leucophthalmus (L.)}

2468-A bacterium isolated from this spittlebug was submitted by $\mathrm{R}$. Whitcomb, Berkeley, California, on July 23, 1965. Identification: Aerobacter sp., group C.

\section{Philaenus spumarius L.}

2792-One, dry mounted adult was submitted by A. G. Wheeler, Ithaca, New York, on December 18, 1967. Diagnosis: Mycosis caused by Entomophthora thaxteriana.

\section{Chermidae}

Chermes picea (Ratz.)

2178-Several specimens, collected at Mt. Mitchell, North Carolina, were submitted by A. T. Drooz, Asheville, North 
Carolina, on October 4, 1963. Diagnosis: Mycosis caused by an unidentified fungus. The fungus which had colonized the insect was no longer viable, and had not produced fruiting structures on the host.

\section{Cicadellidae}

Ballana sp.

2465-A bacterium isolated from this leafhopper was submitted by $\mathrm{R}$. Whitcomb, Berkeley, Califormia, on July 23, 1965. Identification: an Erwinia-like bacterium.

Chlorina furcifera (Horvath) (or Sogata furcifera)

1853-This specimen was submitted by A. D. Hinckley, Fiji, on March 14, 1962. Diagnosis: Mycosis caused by Entomophthora aphidis. New record.

\section{Colladonus montanus (Van Duzee)}

2344-R. Whitcomb, Berkeley, California, submitted several bacterial cultures which had been isolated from $C$. montanus and from celery on December 12, 1964. Diagnosis: Micrococcus sp. and Erwinia sp.

\section{Deltacephalus sp.}

2466-A bacterium isolated from this leafhopper was submitted by $\mathrm{R}$. Whitcomb, Berkeley, California, on July 23, 1965. Identification: Bacillus cereus.

\section{Empoasca devastans Distant}

2620 - A slide containing a fungus isolated from this leafhopper was submitted by R. B. Subba Rao, New Delhi, India, on May 10, 1966. Diagnosis: Mycosis caused by an Entomophthora sp.

\section{Macrosteles fascifrons (Stal)}

2467-A bacterium isolated from this leafhopper was submitted by $R$. Whitcomb, Berkeley, California, on July 23, 1965. Identification: Proteus sp.
2878-On January 23, 1969, C. F. Koval, Madison, Wisconsin, submitted several adults of the aster leafhopper found in the Entomology Department greenhouse. Diagnosis: Mycosis, caused by Beauveria bassiana.

\section{Cicadidae}

Diceroprocta apache Davis

2770-Several preserved nymphs of this cicada were submitted by D. M. Tuttle on August 15, 1967. The specimens were collected near Yuma, Arizona. Diagnosis: Mycosis caused by Cordyceps sobolifera. (Fig. 10.)

\section{Magicicada septendecim (L.)}

1890-Several cicadas were submitted by A. Gray, New York, N.Y., on June 14, 1962. They were infected with a fungus, but were still alive. Diagnosis: Mycosis caused by Massospora cicadina Peck.

1933 - A single specimen collected in Pilot Mound State Forest, Boone County, Iowa, was submitted by $\mathbf{M}$. Lloyd, Los Angeles, California, on August 24,1962 . No microbial etiology was found. Diagnosis: probably non-microbial.

\section{Coccidae}

\section{Nipaecoccus vastator}

2616 - A culture of a fungus isolated from this insect was submitted by R. B. Subba Rao, New Delhi, India, on May 10, 1966. Identification: Fusarium sp. (This fungus was tested against Galleria larvae with negative results.)

\section{Pseudococcus njalensis Laing} 2340 - A fungus culture isolated from $P$. njalensis was submitted by J. T. Legg, Tafo, Ghana, November 18, 1964. Diagnosis: Non-entomogenous fungus, Cephalosporium sp.

Coccus sp.

$2280 \mathrm{~A}$-Some specimens were sub- 


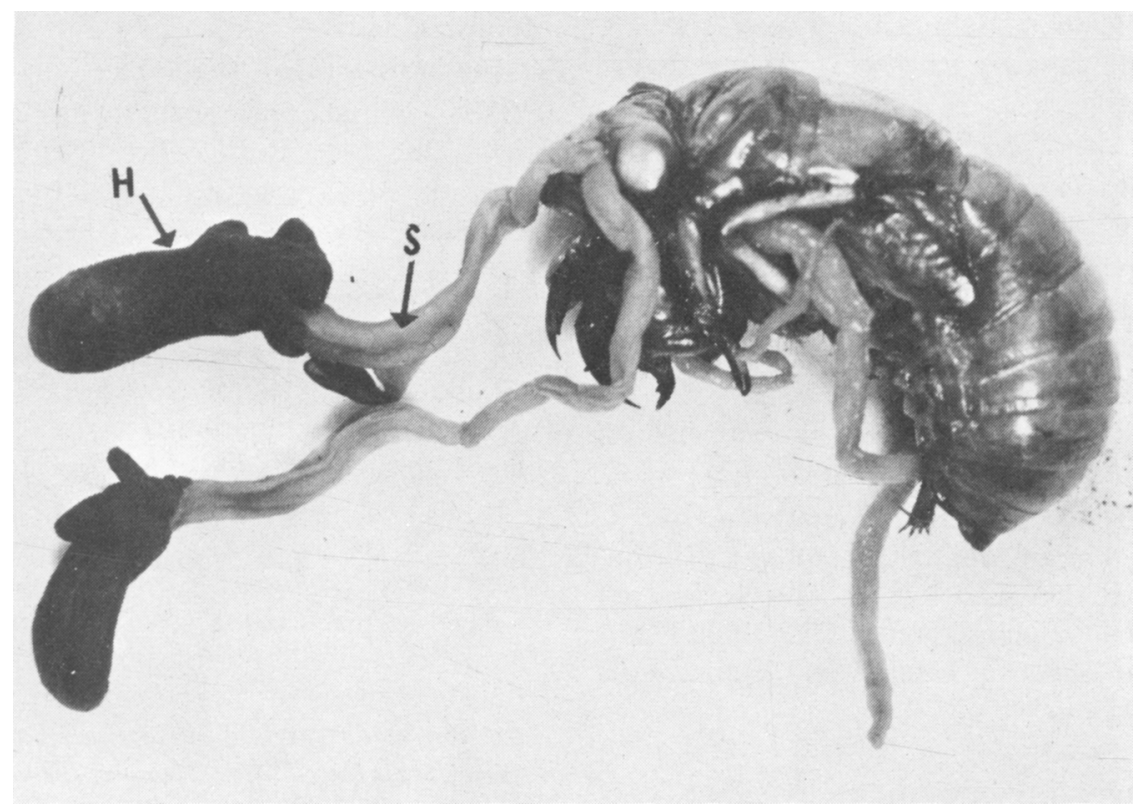

Fig. 10. Acc. No. 2770: A Diceroprocta apache nymph infected with Cordyceps sobolifera, showing mature stroma (S) bearing the fertile spore-producing "head" $(\mathrm{H})$. Magnification= $3.5 \mathrm{X}$.

mitted by $\mathrm{P}$. Somar Sekhar, Mysore State, India, on June 19, 1964. Diagnosis: Non-microbial.

2359-Several specimens were submitted by S. Rao, Kuala Lampur, Malaysia, on February 15, 1965. Diagnosis: Mycosis caused by Fusarium sp.

\section{Saissetia nigra (Nietn.)}

2358-These specimens were submitted by S. Rao, Kuala Lampur, Malaysia, on February 15, 1965. All three groups of specimens suffered from mycoses caused by different fungi. Diagnosis: Mycoses caused by Paecilomyces farinosis (syn. Spicaria farinosa), Fusarium sp., and Sphaerostilbe sp.

\section{Saissetia oleae (Bernard) \\ 2540-Four dead specimens were submitted by C. S. Davis, Berkeley, California, on December 14, 1965. Diag- nosis: Non-microbial.}

\section{Delphacidae}

Nilaparvatalugens (Stal)

1852-This specimen was submitted by A. D. Hinckley, Fiji, on March 14, 1962. Diagnosis: Mycosis caused by Entomophthora sp. New record.

2275, 2293-These specimens were submitted by M. J. MacQuillan, Honiara, Guadalcanal, on June 15 and July 8, 1964. Diagnosis: Mycosis caused by Hirsutella citriformis.

\section{Sogatella furcifera}

2275, 2293-These specimens were submitted by M. J. MacQuillan, Honiara, Guadalcanal, on June 15 and July 8, 1964. Diagnosis: Mycosis caused by Hirsutella citriformis.

\section{Diaspididae \\ Aonidiella aurantii (Maskell)}

2615-A fungal culture isolated from this insect was submitted by $R$. B. Subba Rao, New Delhi, India, on May 
10, 1966. Diagnosis: Fusarium sp. (Tests against Galleria larvae were negative.)

\section{Aspidiotus destructor Sign.}

2797-Several of these scales were received on banana leaves from C. S. Stephens, Changuinola, Panama, on December 29, 1967. Diagnosis: Nonmicrobial.

\section{Lepidosaphes beckii (Newman)}

2078-These specimens were intercepted at the Plant Quarantine Station, San Pedro, California, and submitted by D. M. Peterson on July 2, 1963. Diagnosis: Mycosis caused by Myiophagus ucrainicus.

\section{Phenacaspis pinifoliae (Fitch)}

2945-Several specimens of the pine needle scale were received from $D$. G. Nielsen, Ithaca, New York, on April 3, 1970. The submitted specimens exhibited a scurfy appearance which Mr. Nielsen felt was caused by a fungus. Examination showed that this conidtion was caused by a Cladosporium sp., and an Alternaria sp. A Penicillium sp. was also present. Diagnosis: Saprophytic fungi growing on dead scales. The primary cause of death is unknown.

\section{Flatidae}

\section{Siphanta acuta Melichar}

2405-These torpedo bugs were submitted by R. T. Cunningham, Hilo, Hawaii, on May 13, 1965. Diagnosis: Mycosis caused by Hirsutella citriformis.

\section{Jassidae}

\section{Nephotettix sp.}

2352-Some adults were submitted by G. Rothschild, Kuching, Sarawak, Malaysia, on January 25, 1965. Diagnosis: Mycosis caused by Entomophthora sp.

\section{Membracidae}

Telingana varipes (Walker)

2303-These specimens were submitted by G. Rothschild, Kuching, Sarawak, Malaysia, on July 23, 1964. Diagnosis: Mycosis caused by Isaria sp.

\section{Pseudococcidae}

\section{Mealybug}

1833-These specimens were collected in Cambodia and submitted by J. L. Nickel of the United States Operations Mission to Cambodia on January 15, 1962. Diagnosis: Mycosis caused by Fusarium episphaeria form coccophila. 2317-A. Attafuah, Tafo, Ghana, submitted fungi and bacteria isolated from mealybugs on September 11, 1964 for identification. Diagnosis: Fungi, Verticillium sp. and Neurospora sp.; bacteria, Pseudomonas aeruginosa and Bacillus megaterium; a yeast was also present.

\section{Trionymus sacchari (Cockerell)}

1893-These specimens were submitted by H. E. Fernando, Peradeniya, Ceylon, on June 26, 1962. Diagnosis: Mycosis caused by Aspergillus parasiticus. Passible new host.

\section{Psyllidae \\ Mesohomotoma tessmanni (Alum.)}

2751-Several nymphs were submitted by N. Lodos, Tafo, Ghana, on May 11, 1967. Diagnosis: Mycosis: $E n$ tomophthora sp. (Fig. 11).

\section{Psila rosae (Fabricius)}

2723-Several adults were received from E. Naton, Munich, West Germany, on February 9, 1967. Diagnosis: Nonmicrobial.

\section{Tettigellidae}

\section{Draeculacephala sp.}

2348-Several adults were submitted by W. G. Genug, Belle Glade, Florida, on January 11, 1965. Diagnosis: Mycosis: Entomophthora sp. 


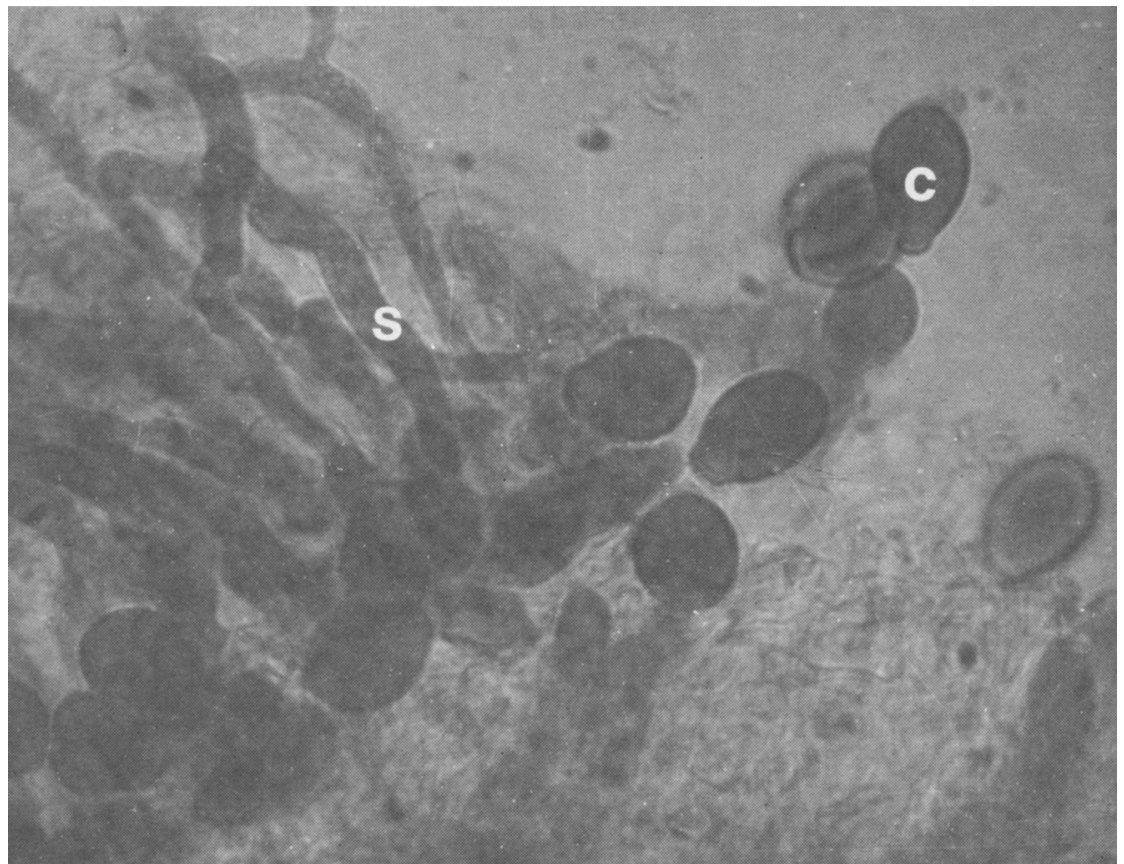

Fig. 11. Acc. No. 2751 : Sporophores (S) and conidia (C) of Entomophthora sp. dissected from Mesohomotoma tessmanni. Magnification $=800 \mathrm{X}$. (Guegen-stained.)

\section{Unidentified}

\section{Frog Hopper}

2928-Agar slant cultures of an entomophagous fungus, isolated from adult frog hoppers, were submitted by $M$. Anarca Ruano, Mexico City, Mexico, on December 5, 1969. Diagnosis: The fungus was identified as Metarrhizium anisopliae.

\section{HYMENOPTERA}

\section{Anthophoridae}

Peponapis angelica (Cockerell)

1863-A specimen was submitted by P. D. Hurd, Berkeley, California, on April 18, 1962. The specimen came from a museum collection and contained a white growth. Diagnosis: No pathogens isolated.

\section{Peponatis pruinosa (Say)}

2944-Several diseased larvae were submitted by Y. Tanada, Berkeley, Cali- fornia, on April 3, 1970. Diagnosis: Probably non-microbial, only a Fusarium sp. and a Micrococcus sp. were isolated.

\section{Apidae}

A pis mellifera (L.)

1856-Several specimens were submitted from hives by $H$. Len Foote, Sacramento, California, on March 19, 1962. Examination revealed that death was probably caused by environmental stress rather than by a microorganism. Diagnosis: Non-microbial.

2479_-In September, 1965, a condition called Autumn Collapse of honeybees was reported by W. Stanger, Davis, California, and H. L. Foote, Sacramento, California. Extensive studies were made of the microbiology of infected hives and bees during four seasons along with nutritional studies. It was concluded that the condition was not a microbial disease, but a nutritional 
deficiency. In certain locations in northern California in the fall when flowers are scarce bees collect secretions from oak galls. This so-called oak "honey dew" lacked some nutritients that were present in flower nectar, resulting in the collapse of healthy, vigorous hives. Supplemental feeding with drivert and wheast evidently solved the problem. Diagnosis: Non-microbial.

2640-Several adult bees were received from H. L. Foote, Sacramento, California, on June 23, 1966. Diagnosis: Non-microbial.

2812-One honeybee larva was submitted by H. L. Foote, Sacramento, California, on March 11, 1968. Diagnosis: Virosis caused by the sacbrood virus, with secondary bacterial deterioration by Aerobacter liquefaciens and a Proteus sp.

2834-Two mummified larvae were submitted by H. L. Foote, Sacramento, California, on May 31, 1968. The larvae had been collected in Tehama Co., California. Diagnosis: Mycosis caused by Ascosphaera apis. This is the first incidence of $A$. apis on honeybees in the United States. (Fig. 12.) (Thomas and Luce, 1972.)

2836-H. L. Foote submitted a larva on June 4, 1968 from Orange County, California. Diagnosis: Bacteriosis, European foulbrood, caused by Bacillus alvei, with associated Streptococcus apis.

\section{Braconidae}

Bracon hebetor (Say)

1975 - Several laboratory-reared specimens were submitted by $R$. C. von Borstel, Oak Ridge, Tennessee, on October 26, 1962. Diagnosis: Cause of death undetermined; may be a lethal mutant.

\section{Macrocentrus sp.}

2904-Several laboratory-reared pupae of this parasite were submitted on July 30, 1969, by D. Brinkman, Albany,
California. Diagnosis: Protozoan infection by Mattesia dispora.

\section{Opius concolor Szepl.}

2335-Pupae of this parasite were submitted by P. Genduso, Palermo, Italy. Diagnosis: Mycosis caused by Aspergillus flavus Link., with secondary bacterioses consisting of Streptococcus faecalis, and a Proteus sp.

\section{Cimbicidae}

\section{Cimbex americana americana (Leach)}

2434 Specimens of the elm sawfly were submitted by J. D. Stein, Fargo, North Dakota, on July 21, 1965. Diagnosis: Bacteriosis caused by Bacillus cereus. New Record.

2627-_Two larvae were received from J. D. Stein, Fargo, North Dakota, on May 31, 1966. Diagnosis: Mycosis: unidentified fungus.

2958-Several dead larvae of the American elm sawfly collected from Siberian elm were submitted on August 6,1970 , by J. D. Stein, Bottineau, North Dakota. Diagnosis: Mycosis caused by a Fusarium sp. This fungus was tested against Galleria larvae for pathogenicity, and was found to be 70 per cent effective.

\section{Cephidae}

Cephus cinctus (Norton)

1952-Several specimens were submitted by R. D. Frye, Fargo, North, Dakota, on September 25, 1962. Diagnosis: Undetermined.

\section{Diprionidae \\ Diprion similis (Htg.)}

2801-Several fungal cultures isolated from this sawfly were received from H. C. Coppel, Madison, Wisconsin, on January 22, 1968. Diagnosis: Two cultures of Beauveria bassiana; a Fusarium sp.; Monocillium sp.; and one culture containing two fungi, an un- 

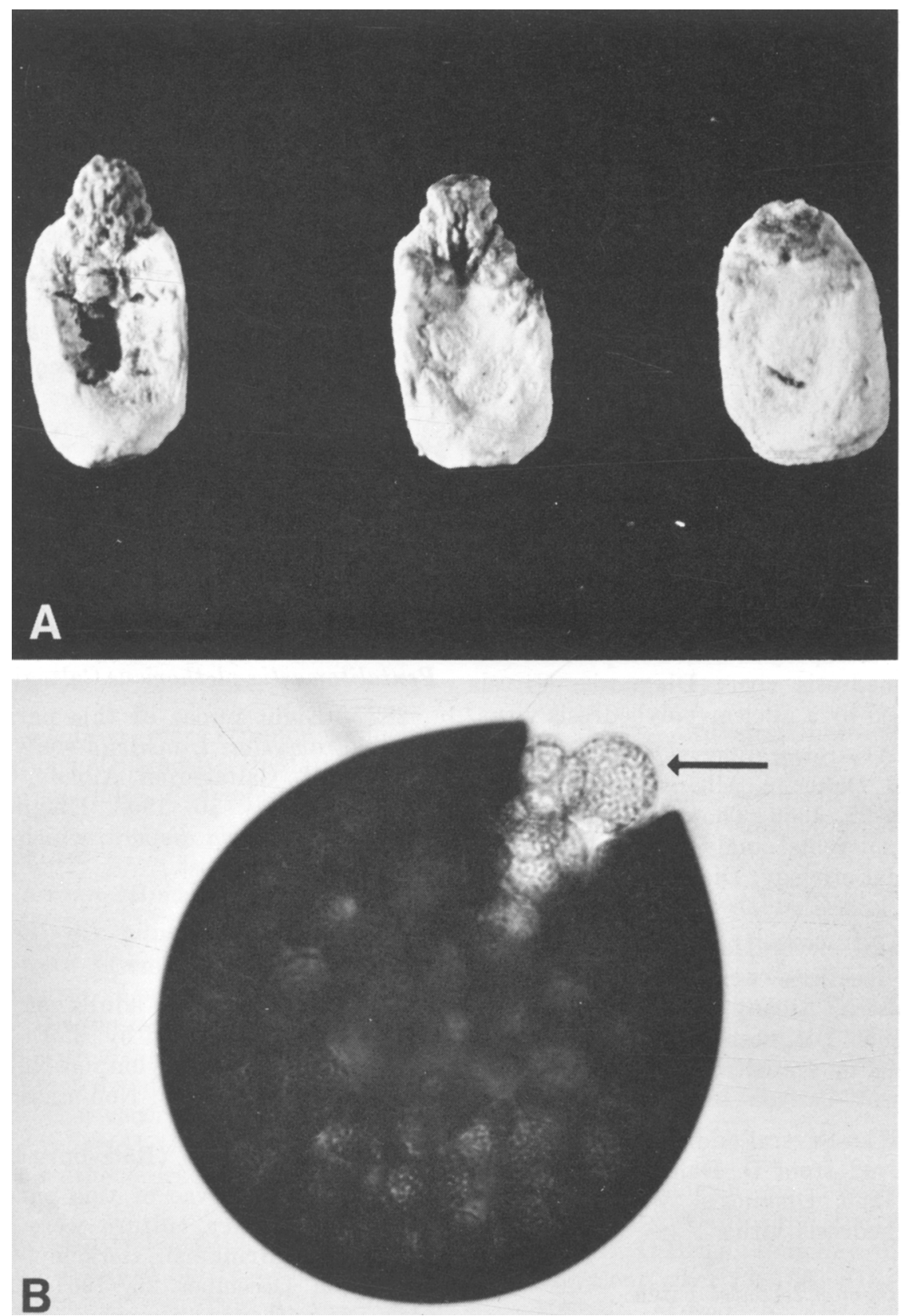

Fig. 12. Acc. No. 2834: Ascosphaera apis, the causal agent of chalk brood in the honeybee Apis mellifera. A: Mummified prepupae covered with chalky appearing fungal mycelium. Magnification $=4 \mathrm{X}$. B : Spore cyst splitting to release spore balls (asci) (arrow) containing ascospores. Magnification $=800 \mathrm{X}$. 
identifiable chlamydospore forming $\mathrm{Hy}$ phomycete overgrown by Rhizopus nigricans.

2820-On April 15, 1968, fungus cultures isolated from this sawfly were received from $\mathrm{H}$. C. Coppel, Madison, Wisconsin. Diagnosis: Fusarium sp.

2863-On October 1, 1968, mounted histological sections, preserved specimens, and dried specimens of sawfly larvae were received from $\mathrm{H}$. C. Coppel, Madison, Wisconsin. Diagnosis: Mycosis caused by an Entomophthora sp.

\section{Neodiprion abietis (Harris)}

2427-Several specimens of the balsam fir sawfly were submitted by D. Dahlsten, Albany, California, on June 29,1965 . The submitted specimens were a sample from a field population which had been sprayed with a suspension of a polyhedrosis virus. Diagnosis: Virosis caused by a nuclear polyhedrosis virus.

2641-Several larvae were submitted by D. Dahlsten, Albany, California, on June 27, 1966. Thorough examination and microbial analysis revealed no microbial etiology. Diagnosis: Non-microbial.

2648--Several larvae and pupae from two localities were submitted by $\mathrm{D}$. Dahlsten, Albany, California, on July 11, 1966. Diagnosis: Chalk Mt.-virosis, caused by a nuclear polyhedrosis virus. Brown's Canyon-non-microbial.

2671-Several additional larvae were received from D. Dahlsten on August 12, 1966. Diagnosis: Virosis: nuclear polyhedrosis virus.

\section{Neodiprion lecontei Fitch}

2319-Several specimens were received on September 18, 1964 from R. C. Wilkinson, Gainesville, Florida. The cadavers had been dried under incandescent lamps, and we were unable to recover any microorganisms. Diagnosis: Undetermined.

\section{Neodiprion merkeli (Ross)}

2706-Several larvae and pupae were received from R. C. Wilkinson, Gainesville, Florida, on November 22, 1966. Diagnosis: Polyhedrosis virus, probabìy cytoplasmic. On December 22, 1966, additional specimens were examined and cytoplasmic polyhedrosis was confirmed.

\section{Neodiprion sp. (probably abietis)}

2648-Several larvae and cocoons of this sawfly were collected at Chalk Mountain and Brown's Canyon, California, and submitted by D. Dahlsten, Albany, California, July 11, 1966. Diagnosis: Chalk Mountain-virosis caused by a nuclear polyhedrosis virus; Brown's Canyon-non-microbial.

\section{Encertidae}

Pentalitomastix plethoricus Caltagirone 2827-Eight pupae of this parasite from Paramyelois transitella were submitted by L. Caltageroni, Albany, California, on May 10, 1968. Diagnosis: Protozoan-Mattesia dispora, which also had infected the host.

\section{Eulophidae}

Dimmockia javanica Ferr.

3034-Several dead adults of this parasite were submitted by H. E. Fernando, Peradeniya, Ceylon, on November 24, 1971. Diagnosis: Non-microbial.

\section{T'etrastichus incertus (Ratzeburg)}

2795-Three larvae of this parasite from an insectary culture were submitted by D. Brinkman, Berkeley, California, on December 26, 1967. Diagnosis: No pathogens present.

\section{Eurytomidae}

\section{Eurytoma amygdali}

2926-Several deteriorated larvae were received from H. N. Plaut, Haifa, Israel, on November 21, 1969. The lar- 


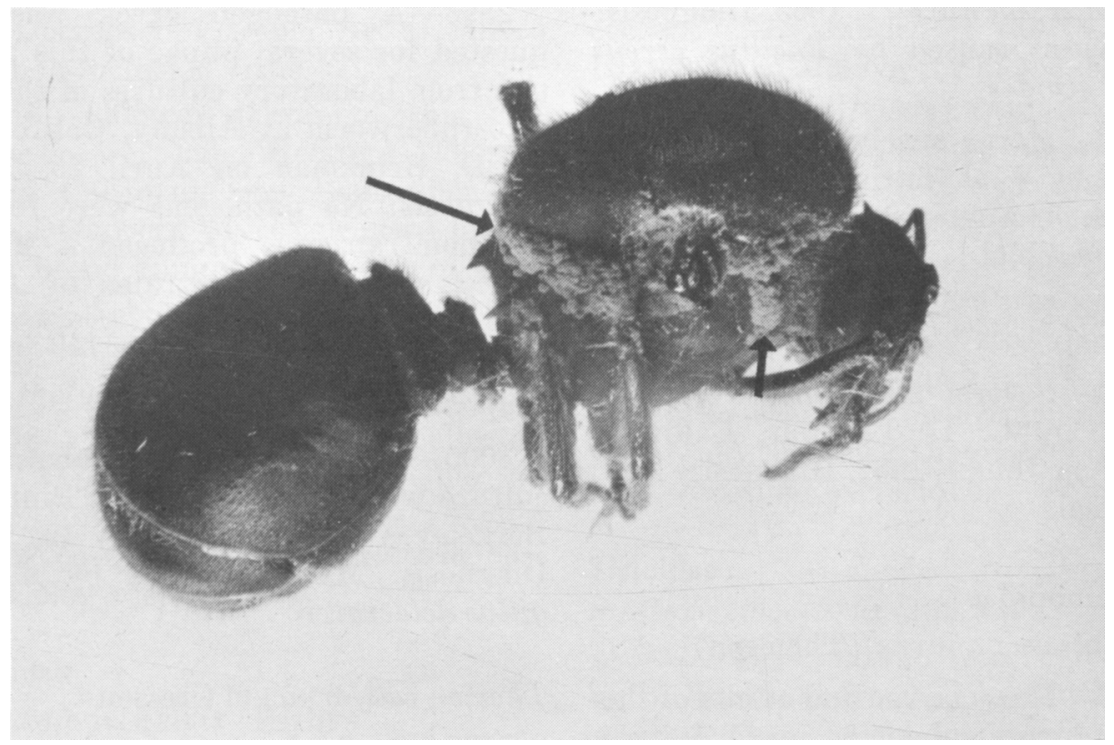

Fig. 13. Acc. No. 1888: Adult Atta texana infected with Aspergillus flavus. Note sporulating fungus protruding from dorsal thoracic segments and around the cervix (arrows). Magnification $=8 \mathrm{X}$.

vae had been reared under artificial conditions. Diagnosis: Predation by a predatious mite, Pyemotes ventricosus.

\section{Formicidae}

\section{Atta texana (Buckley)}

1888-Six adult queen leaf-cutting ants were submitted by J. C. Moser, Alexandria, Louisiana, on June 11, 1962. Diagnosis: Mycoses; 5 were infected with Aspergillus flavus, and one was infected with Beauveria bassiana. A new host record for both fungi. Dr. Moser reported that only the queens, and not the workers, were infected. (Fig. 13.)

1972-On October 16, 1962, 5 adult major workers of the Texas leaf-cutting ant were received from J. C. Moser. Diagnosis: Mycosis caused by Beauveria bassiana. This is the first time this fungus has been reported from the workers of this ant.

\section{Solenopsis saevissima (F. Smith)}

1985-Two dead adult fire ants were received from A. Silveira-Guido, Montevideo, Uruguay, on December 7, 1962. Diagnosis: Mycosis: Metharrizium anisopliae; a new host record for this fungus.

Solenopsis saevissima richteri Forel

2326-Three specimens of the imported fire ant were received on October 8, 1964 from A. R. Khanm, State College, Mississippi. The ants were collected in Florida. Diagnosis: Mycosis caused by Aspergillus flavus Link.

\section{Unidentified ant}

2164 This specimen was collected in Tehama County, California, and submitted by L. O'Brien, Berkeley, California, on September 13, 1963. Diagnosis: Mycosis caused by Beauveria bassiana.

\section{Halictidae}

Lasioglossus (Sphecodegastra) aberrans (Crawford)

2438-Several specimens from Antioch, California, were submitted by W. 
J. Turner, on July 27, 1965. Diagnosis: Bacteriosis caused by Bacillus cereus var. mycoides.

2461-More specimens were submitted by W. J. Turner, Berkeley, California, on August 24, 1965. Diagnosis: Non-microbial.

\section{Nomia sp.}

1881-Several specimens were submitted by C. D. Bechtolt, Caldwell, Idaho, on June 1, 1962. Diagnosis: Nonmicrobial.

\section{Ichneumonidae}

\section{Bathyplectus anurus (Thomson)}

3024-Many larvae and adults of this parasite were submitted by C. Legace, Berkeley, California, on November 3, 1971. They were collected from sting cages, where they were parasitizing alfalfa weevil larvae. The submitted material also contained parasitized dead alfalfa weevil larvae with undeveloped parasites. Diagnosis: Bacteriosis caused by Streptococcus faecalis var. liquefaciens. Evidently, the adult parasites were contaminated with the bacterium and inoculated the host larvae during oviposition, making them unsuitable for development of the parasite.

\section{Diadegma molesta Uchida}

2680-Several adults, larvae, and pupae of this parasite were submitted by D. Brinkman, Berkeley, California, on September 6, 1966. Diagnosis: bacteriosis: Streptococcus faecalis var, liquefaciens.

2875-On December 27, 1968, D. Brinkman, Albany, California, submitted eleven pupae of this tuberworm from his laboratory cultures. Diagnosis: Bacterioses caused by the potential pathogens Bacillus cereus and Streptococcus faecalis. Infection by these potential pathogens may be indicators of some other predisposing stress factors.
2893-A pathogen check was requested for several pupae of this parasite from laboratory cultures of the potato tuberworm at Albany, California, by D. Brinkman on April 23, 1969. Diagnosis: No pathogens were found. Microbial analysis produced a Micrococcus sp., and two strains of Aerobacter liquefaciens.

\section{Hypogaster fugitivus (Say)}

3000 - Three adults from a laboratory culture were submitted by D. Pinnock, Berkeley, California, on July 19, 1971. Diagnosis: Mycosis caused by Aspergillus delacroixii.

\section{Therion californicum Cresson}

2528-A single dead specimen was submitted by L. Etzel, Berkeley, California, on September 28, 1965. The parasite was dissected from a Heliothis zea pupa which had been collected in the field as a larva and reared in the laboratory on an artificial diet. Diagnosis: Mycosis caused by a Fusarium sp.

\section{Megachilidae}

\section{Megachile rotundata (Fabricius)}

2446-Several larvae of this leaf cutting bee were submitted by $R$. W. Thorpe, Davis, California, on July 29, 1965. Diagnosis: Bacteriosis caused by Bacillus cereus. Normally a soil saprophyte, $B$. cereus, can cause septicemia in insects that have been weakened by some other predisposing factor.

2473-Additional larvae of this bee were submitted by R. W. Thorpe, Davis, California, on September 20, 1965. Some of the specimens had been parasitized. All had molds growing on them. Diagnosis: Aspergillus niger, A. sulphureus, A. fumigatus, A. glaucus and Rhizopus nigricans.

2657-On July 27, 1966, R. W. Thorpe, Davis, California, submitted several more larvae of this leaf-cutting 
bee. Diagnosis: Possible virosis caused by a non-inclusion virus.

3016-Thirteen larvae of this leaf cutter bee were submitted by $R$. Thorpe, Davis, California, on September 20, 1971. Diagnosis: Mycosis caused by the chalk brood fungus, Ascosphaera apis.

\section{Pamphilidae}

\section{Acantholyda luteomaculata (Cresson)}

2276-Five prepupae were collected from white pine in Newark, Ohio, by W. R. Holt, on June 15, 1964. Diagnosis: Mycosis caused by Beauveria bassiana. New record.

\section{Pergidae}

\section{Perga affinis affinis (Kirby)}

2153-Several specimens were submitted by P. B. Carne, Canberra, Australia, on September 4, 1963. Diagnosis: Mycoses caused by Metarrhizium anisopliae and Beauveria bassiana. New record-new host.

\section{Polistidae}

\section{Polistes olivaceus (DeGeer)}

1851-One specimen was submitted by A. D. Hinckley, Fiji, on March 14, 1962. Diagnosis: Mycosis caused by Hirsutella saussurei.

\section{Scelionidae}

\section{Telenomus alsophila Vier}

3006-A single adult parasite was submitted by G. F. Fedde, Research Triangle Park, North Carolina, on August 20,1971 . The specimen had been reared in a closed vial with a cotton disc saturated with honey water. Diagnosis: Possible mycosis, or at least a secondary infection by Aspergillus ochraceous.

\section{Scoliidae}

\section{Scolia procer Illiger}

2234 - Twenty cocoons were received from R. P. Owen, Koror, Palau, Caroline Islands, on February 19, 1964. The cocoons were shipped to the Caroline Islands from Malaya for biological control studies of Oryctes rhinoceros. In three separate shipments, all but one specimen arrived dead. The condition of the specimens certainly indicated disease, but no microbial etiology was determined. However, there was a bacterium morphologically resembling a Clostridium present in all cadavers. Attempts to isolate it on artificial media were unsuccessful. Diagnosis: Indefinite-possible bacteriosis.

\section{Siricidae}

Syntexis libocedrus (Rohwer)

2311-These larvae were submitted by B. Wickman from Viola, California, on September 8, 1964. Diagnosis: Probably in diapause.

\section{Rhyssa sp.}

2534-Fifteen dead specimens were submitted by F. Wilson, Ascot, Berks, England, on December 6, 1965. Diagnosis: A-Streptococcus faecalis var. liquefaciens; B-no microbial etiology; C-fungus Stysanus sp.; D-same as in $\mathrm{C}$; E-same as in $\mathrm{C}$ and a Verticillium sp.; F-Stysanus sp. plus 2 other saprophytic fungi, a Trichoderma sp. and a Penicillium sp.; G-Penicillium sp. and a Gliocladium sp.; H-saprophytic Verticillium sp.; acarid mites and Pseudomonas aeruginosa; I-Stysanus sp., Verticillium sp., Pseudomonas aeruginosa, and aphelenchoid nematodes; Jsame as in I; K-Stysanus sp.; L-Penicillium sp. and Gliocladium sp.; Msame as in $\mathrm{L}$; $\mathrm{N}$-Streptococcus sp., probably a potential pathogen; $\mathrm{O}-$ Penicillium sp.

\section{Tenthredinidae}

\section{Athalia proxima Klug}

2546-Two bacterial cultures isolated from this sawfly were submitted by B. K. Srivastava, Udaipur, India. Diagnosis: Bacteria identified as Serratia mercescens in both cultures. 
Fenusa ulmi (Sunderall)

1884-These specimens were submitted by R. D. Frye, Fargo, North Dakota, on June 1, 1962. Diagnosis: Non-microbial.

\section{Pontania bozemani Cooley}

2963-Several dead larvae of this sawfly were submitted by J. Stein, Bottineau, North Dakota, on September 8, 1970. The larvae were collected from Populus balsimifera; Bottineau County, North Dakota, where the insect population averaged five larvae per tree, and about 40 per cent of the population was affected. Examination revealed parasitization by a hymenopterous parasite. Diagnosis: Non-microbial. Death caused by an insect parasite.

\section{Pristophora erichsonii (Hartig)}

1835-One dead and dried-out prepupa of the larch sawfiy was submitted by A. T. Drooz, Asheville, North Carolina, on January 20, 1962. The specimen had been collected in Renova, Pennsylvania, in August, 1961. Diagnosis: Mycosis caused by Paecilomyces farinosus. A new host record for this fungus.

1934-Some specimens collected in Pennsylvania and New York were submitted by A. T. Drooz, Asheville, North Carolina, on August 27, 1962. Diagnosis: Mycoses caused by Beauveria bassiana and Paecilomyces farinosus.

2047-Some specimens collected in Pennsylvania were submitted by A. T. Drooz on April 27, 1963. Diagnosis: Mycoses caused by Beauveria bassiana, Paecilomyces farinosus and Penicillium sp.

2177-Additional specimens collected at the Tomhannock Reservoir, Rennselaer County, New York, were submitted by A. T. Drooz on October 4, 1963. Of the 77 specimens, twelve prepupae were selected and found to be infected with fungi. Diagnosis: Mycoses caused by
Beauveria bassiana and Paecilomyces farinosus.

2322-Specimens collected in Renovo, Pennsylvania, were submitted by A. T. Drooz on October 1, 1964. Diagnosis: Mycosis caused by Beauveria bassiana.

2864-Thirty-seven prepupae of the larch sawfly were submitted by A. T. Drooz, Research Triangle Park, North Carolina, on October 2, 1968. Diagnosis: Non-microbial. Several saprophytic fungi were isolated including: Verticillium sp. Penicillium sp., Rhizopus nigricans, and a Monosporium sp.

\section{Profenusa mainensis}

2708-Several larvae were received from L. J. Lipousky, Augusta, Maine, on December 5, 1966. Diagnosis: Mycosis caused by Paecilomyces farinosus.

\section{Unidentified Sawfly}

2895-Several larvae of a laboratoryreared sawfly were submitted on June 18, 1969 by J. R. Milodragovich, Missoula, Montana, with a request for a virus check. Diagnosis: Non-microbial.

\section{Vespidae}

Vespula maculata (L.)

1882_-One specimen was submitted by R. D. Frye, Fargo, North Dakota, on June 1, 1962. Diagnosis: Mycosis caused by Beauveria bassiana. (Fig. 14.)

\section{Unidentified}

Bee

2724-Three adult bees were received from B. Meksongsee, Bangkhen, Bangkok, Thailand, on February 9, 1967. Diagnosis: Possible mycosis caused by a Fusarium sp.

\section{Wasp}

2137-An agar slant of a culture isolated from a wasp was submitted by $\mathrm{J}$. 


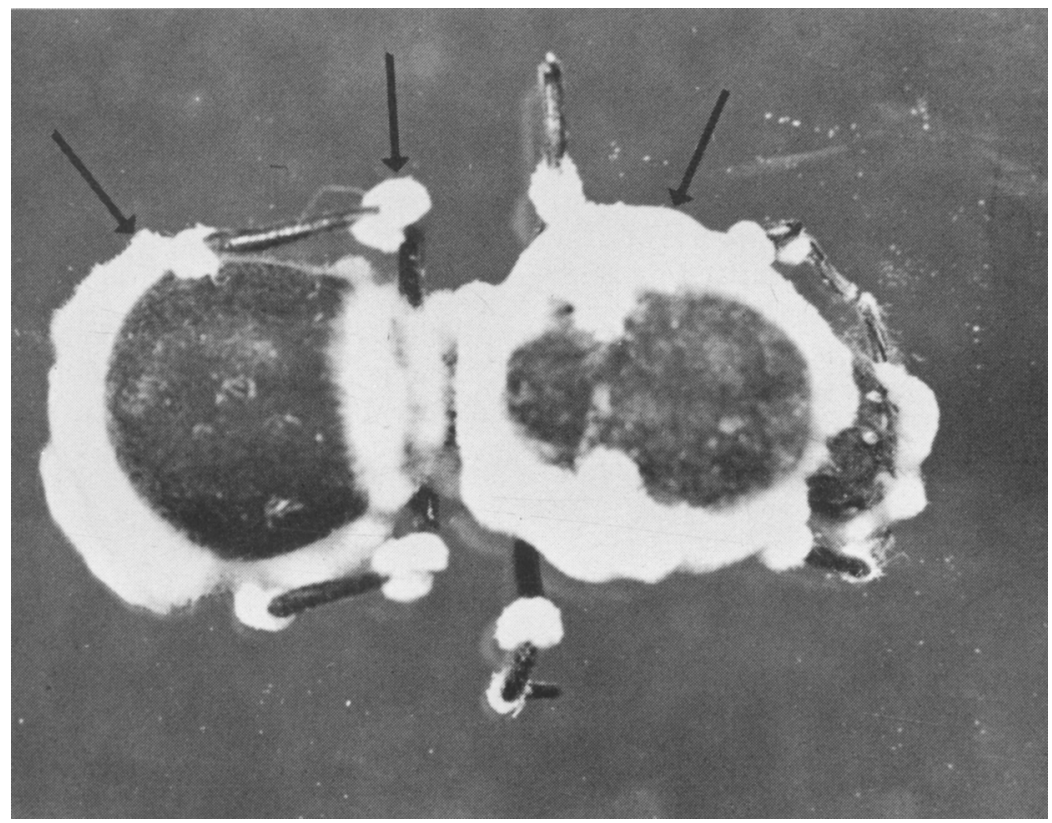

Fig. 14. Acc. No. 1882: Adult Vespula maculata infected with Beauveria bassiana. Fungus protrudes from mummified host between sclerites and leg joints (arrows). Magnification $=5 X$.

C. Basu Choudhuri, Ciombatore, India, on August 22, 1963. Identification: Isaria sp.

\section{Wild Bee}

2626-One pupa was received from B. J. Donovan, Auckland, New Zealand, on May 27, 1966. Diagnosis: Mycosis caused by Ascosphaera (Pericystis) alvei.

2946 - Twelve adult wild bees received for taxonomic study were submitted by H. V. Daly on April 17, 1970. Diagnosis: Non-microbial. Saprophytic fungi included Cladosporium sp., Rhizopus nigricans, and a Penicillium sp.

\section{ISOPTERA}

\section{Rhinotermitidae}

Reticulotermes flavipes Kollar

2308-Several dried and preserved specimens were received from $R$. $H$. Beal, Gulfport, Mississippi, on August
17, 1964. The insects died in sawdust cultures in the laboratory. Diagnosis: Probably non-microbial.

2400 - Several laboratory-reared specimens were submitted by $R$. V. Smyth, Madison, Wisconsin, on April 28, 1965. Diagnosis: Primary etiology undetermined, but with a secondary bacteriosis caused by Aerobacter sp.

\section{Unidentified Termites}

2823-Several adult termites were submitted by D. McKay, Redington Shores, Florida, on April 26, 1968. Diagnosis: Non-microbial.

\section{LEPIDOPTERA}

\section{Aegeriidae}

Bembecia marginata (Harris)

2060_This specimen was submitted by N. L. H. Krauss, Corvallis, Oregon, on May 29, 1963. Diagnosis: Mycosis caused by Beauveria bassiana. 


\section{Sanninoidea exitiosa (Say)}

2768-One larva was submitted by G. C. Rock, Raleigh, North Carolina, on August 14, 1967. Diagnosis: Non-microbial.

2857-One mycotic cocoon of the peach tree borer was submitted by S. S. Rosenthal, Fort Valley, Georgia, on August 27,1968 . The specimen was collected from a peach tree 5 miles northwest of Fort Valley on August 12, 1968. Diagnosis: Mycosis caused by Paecilomyces farinosus.

\section{Thannosphecia scitula (Harris)}

1978-A single specimen was submitted by C. D. Pless, Knoxville, Tennessee, on October 31, 1962. Diagnosis: Mycosis caused by Cordyceps sp., possibly $C$. elongata.

\section{Vespamima sequoiae (Hy. Edwards)}

2962_-Several larvae of the sequoia pitch moth from Los Gatos, California were submitted by S. Rosenthal on September 1, 1970. Diagnosis: Non-microbial.

\section{Vitacea polistiformis (Harris)}

2282-These specimens were field collected from vineyards in Northwest Arkansas and Southwest Missouri and submitted by R. L. Combs, Fayetteville, Arkansas, on June 29, 1964. Diagnosis: Mycosis caused by Metarrhizium anisopliae.

2844-Several mummified larvae of the grape root borer from Mountain Grove, Missouri, were received on June 26, 1968 from D. Singh Sarai. Diagnosis: Mycoses caused by Beauveria bassiana and Metarrhizium anisopliae.

Vitacea polistiformis polistiformis (Harris)

2866-Fungus cultures isolated from grape root borer larvae were submitted on October 14, 1968, by E. P. Mason, Fayetteville, Arkansas. Diagnosis: Fungus identified as Metarrhizium anisopliae.

\section{Arctiidae}

Antarctia sp.

2296-S. Shima, Jr., Palmira, Valle, Colombia, submitted several dead larvae on July 10, 1964. Diagnosis: bacteriosis probably caused by Streptococcus sp.

\section{Diacrisia obliqua Wlk.}

2934-Seven dead larvae were received from O. S. Bindra; Ludhiana, India, on February 10, 1970. Diagnoses: Two suffered a mycosis caused by $A s$ pergillus flavus; three suffered a bacteriosis caused by the potential pathogen Streptococcus faecalis, and one suffered a virosis caused by a granulasis virus. The last was non-microbial.

\section{Ecpantheria sp.}

2443-These specimens were submitted by F. Lara, Limon, Costa Rica, on July 28, 1965. Diagnosis: Primary etiology obscured by the action of acarid mites.

\section{Estigmene acrea Drury}

2916-_On September 19, 1969, many larvae of the salt marsh caterpillar were submitted by L. Falcon, Berkeley, California. Diagnosis: Mycosis caused by Entomophthora grylli.

2983-A larva of the salt marsh caterpillar was submitted by G. Leon Quant, Leon, Nicaragua, on February 3, 1971. The specimen was collected in the field, and reared in the laboratory. Diagnosis: Mycosis caused by an Entomophthora sp.

2995-A larva of the salt marsh caterpillar was submitted from Maragua, Nicaragua, by L. A. Falcon on May 27, 1971. Diagnosis: Non-microbial. 


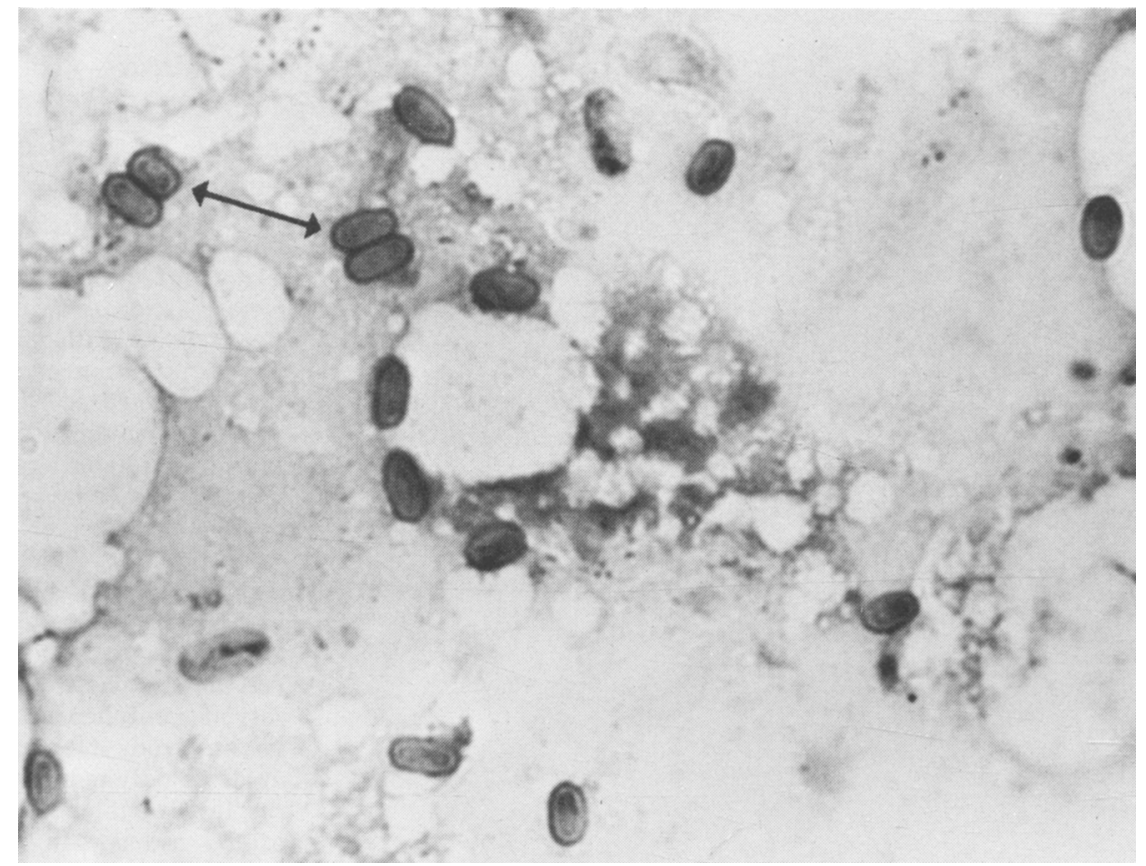

Fig. 15. Acc. No. 2411: Spores (arrows) of a Nosema sp. in the fat body of a Estigmene sp. Magnification $=1600 \mathrm{X}$.

\section{Estigmene sp.}

2411-A larva was submitted by J. Hoy, Berkeley, California, on June 1, 1965. Diagnosis: Microsporidiosis caused by a Nosema sp. (Fig. 15.)

\section{Halisidota argentata (Packard)}

2088-A fungus culture isolated from this insect was submitted by L. W. Getzin, Puyallup, Washington, on July 28, 1963. Diagnosis: Probably Paecilomyces farinosis.

\section{Hyphantria cunea (Drury)}

1932_On August 17, 1962, several dead larvae of the fall webworm were received from the laboratory culture of A. D. Oliver, Baton Rouge, Louisiana. Examination of these specimens and of additional specimens received at a later date showed the presence of two different viruses with some individuals infected by both viruses. Diagnosis:
Viroses; a granulosis and a nuclear polyhedrosis. This is the first record of these two viruses in North America. (Fig. 16.)

\section{Isia isabella (J. E. Smith)}

2782-Several dead larvae of the banded wooly bear caterpillar collected near Albany, New York, were submitted by $H$. A. Jamnback on November 2, 1967. The specimens were very deteriorated by secondary saprophytic fungi. Diagnosis: Non-microbial, or if a primary microbial etiology was present it had been masked by the saprophytic fungi.

\section{Unidentified}

1983-These specimens were submitted by A. Silveira-Guido, Montevideo, Uruguay, on November 26, 1962. Diagnosis: Undetermined. 


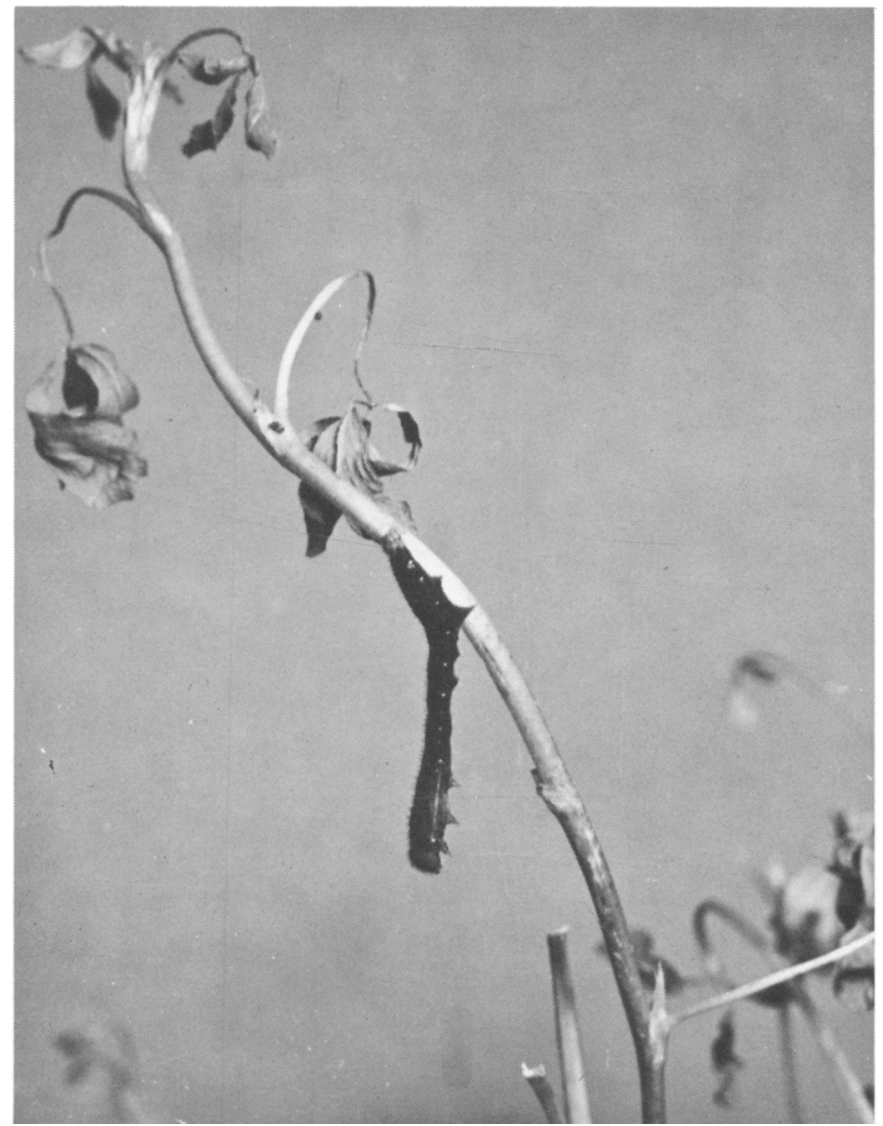

Fig. 16. Acc. No. 1932 :

Virus infection of $H y$ phantria cunea. TOP: $H$. cunea larva killed by a double infection of a nuclear polyhedrosis virus and a granulosis virus. The limp, hanging attitude of the cadaver is a typical symptom of a nuclear polyhedrosis infection. BOTTOM: Electron micrograph showing granulosis virus capsules (C) and a partially dissolved polyhedron revealing rods $(R)$ of the nuclear polyhedrosis virus in a double infection of $H$. cunea. Magnification $=15,600 \mathrm{X}$.

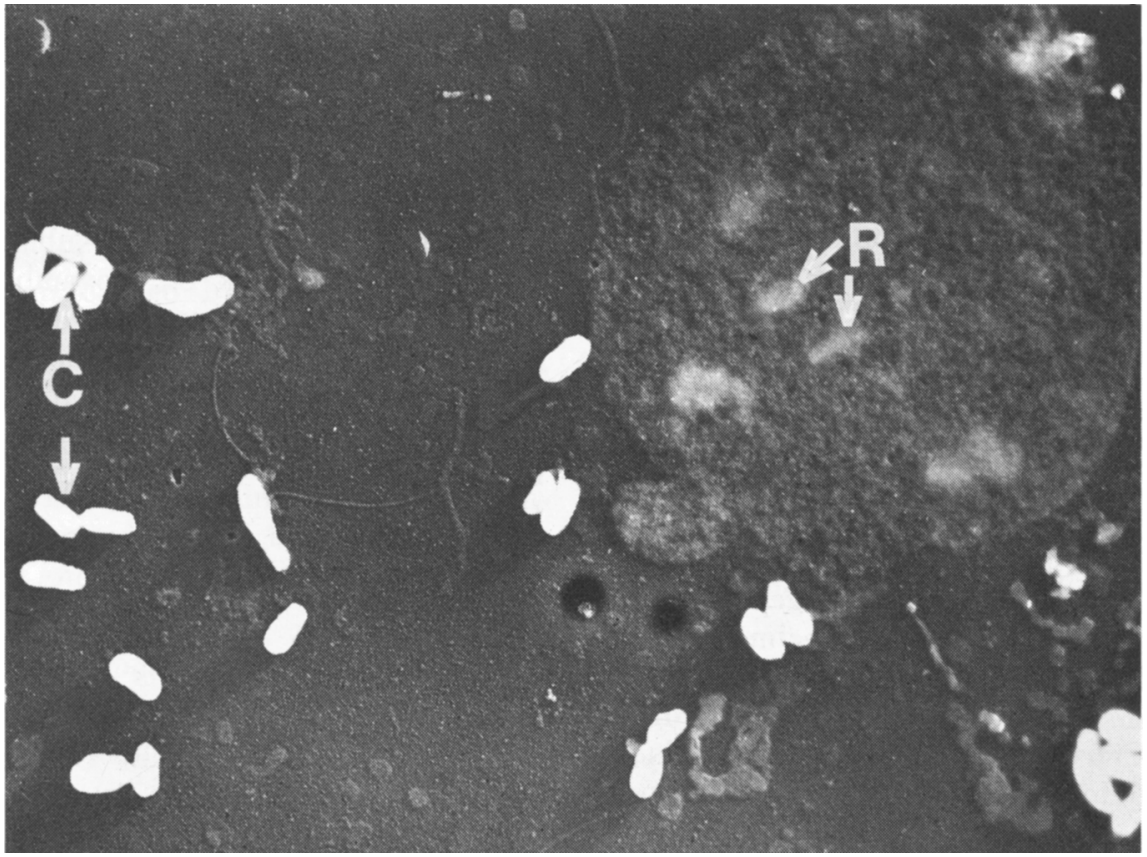


Unidentified

2003-Several specimens were submitted by C. S. Stephens, La Lima, Honduras, on January 19, 1963. Diagnosis: Mycosis caused by Entomophthora aulicae.

\section{Bombycidae}

Bombyx mori Linn.

2342 - Several laboratory-reared specimens were received from I. Gard, Wasco, California, on November 19 and 30, 1964. Most of the submitted specimens were healthy, but one was infected with a bacterium. Diagnosis: Bacteriosis in one specimen caused by a Streptococcus sp.

\section{Castniidae}

Castnia licoides (Boisduval)

2017-Several preserved specimens were submitted by J. F. Bates, Georgetown, British Guiana, on February 19, 1963. Diagnosis: Undetermined.

2256-These specimens were submitted by J. F. Bates, Georgetown, British Guiana, on April 24, 1964. Diagnosis: Mycosis caused by Paecilomyces cossus. New record.

\section{Coleophoridae}

\section{Coleophora parthenica Mey}

2988-Three dead larvae were submitted on April 29, 1971 by R. Hawkes, Albany, California. Larvae of this insect were sent to the United States from Egypt in stems of Salado iberica, and the dead larvae were found when the stems were dissected. Diagnosis: Nonmicrobial.

\section{Cossidae}

\section{Prionoxystus robiniae (Peck)}

2039-Two larvae of the carpenterworm were submitted by J. D. Solomon, Stoneville, Mississippi, on April 9, 1963. They were laboratory-reared on an artificial diet. Diagnosis: Some gram negative, lactose negative small rods, a gram positive, lactose negative, small rod and a gram positive coccus were isolated. They may or may not have been the cause of death.

2260 - One specimen found dead in its entrance hole in overcup oak was submitted by J. D. Solomon, Stoneville, Mississippi, on May 7, 1964. Diagnosis: Bacteriosis: gram negative, non-lactose fermenting, rodshaped bacterium.

2318-Four additional specimens of the carpenterworm were submitted by J. D. Solomon, Stoneville, Mississippi, on September 16, 1964. The submitted specimens were reared on an artificial diet where mortality reached almost 50 per cent in the 5 th and 7 th instars. Diagnosis: Bacterioses caused by Serratia marcescens, with a secondary invader, Alcaligenes faecalis.

2331-Two larvae found dead in their galleries near Stoneville, Mississippi, were submitted by J. D. Solomon on October 26, 1964. Diagnosis: Bacterioses: Streptococcus faecalis, and Escherichia coli. In addition, a yeast and a Verticillium sp. were isolated from the specimens.

\section{Xyleutes ceramicus Walker}

2594-Two dead larvae of the teak beehole borer were submitted by W. W. Cantel, U. S. Operations Mission to Thailand, on April 7, 1966. The specimens were collected in Sukhothai, Thailand, on March 14, 1966. Diagnosis: Mycosis caused by Beauveria bassiana.

\section{Crambidae}

Chilo zonellus (Swinhoe)

2323-Several larvae were submitted by T. V. Venkatraman, Khartoum North, Republic of the Sudan, on October 5,1964 . The disease, apparently disseminated by a dipterous fly, affected about 30 per cent of the experimental 
field population. Specimens were so deteriorated that a positive diagnosis of the original cause of death was not possible. Diagnosis: Probably nonmicrobial. Possibly a dipterous parasite.

2478-Slides of a protozoan collected from this insect were submitted by $R$. B. Subba Rao, New Delhi, India, on September 20, 1965. Identification: A microsporidian.

2610_Several specimens were submitted by R. B. Subba Rao, New Delhi, India, on May 10, 1966. Diagnosis: Bacterioses caused by Bacillus cereus and Streptococcus faecalis var. liquefaciens.

\section{Chilo sp.}

2303-These specimens were submitted by G. Rothschild, Kuching, Sarawak, on July 23, 1964. Diagnosis: Mycosis, caused by Hirsutella barberi.

\section{Diatraea saccharalis (Fabricius)}

2312-Eleven specimens of the sugar cane borer from Montevideo, Uruguay, were submitted by A. Silveira-Guido on September 10, 1964. The specimens were shriveled and quite deteriorated. Diagnosis: Virosis, caused by a granulosis virus. A number of gram negative secondary bacteria and two saprophytic fungi, a Penicillium sp., and a Verticillium sp., also were present.

\section{Diatraea venosata (Walker)}

1943-Bacterial cultures from this species were submitted by Wen-YiCheng, Tainan, Taiwan, on September 12, 1962. Identification: Pseudomonas aeruginosa.

\section{Unidentified sugarcane stem borer}

1859-One specimen was collected in Takwa Ling, Hong Kong, and submitted by V. P. Rao, Bangalore, India, on March 28, 1962. Diagnosis: Possible bacteriosis caused by Serratia marcescens.

\section{Danaidae}

Danaus plexippus (L.)

2436 - Several laboratory-reared specimens were submitted by J. Brewer, Newton Highlands, Massachusetts, on July 26, 1965. They were collected in Ontario, Canada, and reared in Massachusetts. Diagnosis: Bacteriosis caused by Pseudomonas aeruginosa (Brewer and Thomas, 1966.)

2447-Additional specimens were received from J. Brewer, Newton Highlands, Massachusetts, on August 3, 1965. Cause of death was found to be nonmicrobial, with a secondary bacteriosis. Diagnosis: Non-microbial.

2454_Several more laboratory reared specimens were submitted by $\mathrm{J}$. Brewer on August 9, 1965. They had been reared under controlled light waves. Diagnosis: Non-microbial.

2455-More laboratory reared specimens were submitted by J. Brewer on August 10, 1965. Examination showed only a secondary bacteriosis. Diagnosis: Undetermined.

2582-One dead specimen, received from J. Brewer, Newton Highlands, Massachusetts, was collected in the summer of 1963 and submitted on March 15 , 1966, with the request for a virus check. Diagnosis: No virus present.

2716-Several specimens, adults and pupae, were received from Dr. L. P. Bower, Department of Biology, Amherst College, Amherst, Massachusetts, on January 4, 1967. Diagnosis: Probably bacterioses; cultures from externally sterilized and triturated specimens produced only Streptococcus faecalis and Aerobacter grp. C.

2846-Larvae and pupae were submitted by P. Samuelson, Pleasantville, Iowa, on July 5, 1968. These specimens were being reared under artificial conditions. Diagnosis: Non-microbial.

2936-On February 12, 1970, seven larvae from an artificial medium were 
submitted by C. White, Shafter, California. Diagnosis: Bacteriosis caused by the potential pathogen, Streptococcus faecalis.

\section{Ethmiidae}

Ethmia sp.

2258-J. Powell, Berkeley, California, submitted eight dead larvae on May 8,1964 . The specimens were field-collected, and died in the laboratory. Diagnosis: Bacteriosis consisting mainly of Pseudomonas sp.

\section{Galleriidae}

Galleria melonella (L.)

2339_Several larvae were submitted by M. Tamashiro, Berkeley, California, on November 12, 1964. These larvae were laboratory-reared, and had been injected with TIV virus and kept at room temperature. It was found that the TIV suspension was contaminated. Diagnosis: Bacterioses: Proteus sp., Escherichia coli, and Shigella sp.

2532 - Two dead specimens were submitted by W. R. Kellen, Fresno, California, on November 30, 1965. The Galleria supposedly were infected with Spirillum sp. and submitted for confirmation. Diagnosis: No Spirillum infection could be found.

\section{Gelechiidae}

\section{Pectinophora gossypiella (Saunders)}

2614-A fungus culture isolated from the pink bollworm was submitted by R. B. Rao, New Delhi, India, on May 10, 1966. Diagnosis: Saprophytic fungus, Fusidium sp. This fungus was tested against Galleria larvae for pathogenicity with negative results.

\section{Phthorimaea operculella (Zeller)}

1946-Three preserved larvae of the potato tuberworm were received from M. D. deSilva, Gurutalawa, Ceylon, on September 14, 1962. Diagnosis: Virosis caused by a granulosis virus. New host record.

1977-Several laboratory reared specimens were submitted by L. Anderson, Palisade, Colorado, on October 29, 1962. Diagnosis: Microsporidiosis caused by Nosema sp.

2321-Several larvae were submitted by A. Silveira-Guido, Montevideo, Uruguay, on September 22, 1964. Diagnosis: Bacteriosis: Streptococcus sp.

2328-Several laboratory reared specimens of the potato tuberworm were submitted by D. Brinkman, Albany, California, on October 9, 1964. Diagnosis: Bacteriosis caused by Serratia marcescens.

2417-These laboratory-reared larvae were submitted by D. Brinkman, Albany, California, on June 14, 1965. Diagnosis: Bacterioses caused by Streptococcus faecalis and Aerobacter sp.

2876-Several laboratory-reared larvae were submitted by L. Anderson, Palisade, Colorado, on January 13, 1969. Diagnosis: Non-microbial.

2888-Some laboratory-reared larvae of the potato tuberworm were submitted by L. Anderson, Palisade, Colorado, on March 27, 1969. Diagnosis: Bacteriosis caused by the potential pathogen Streptococcus faecalis var. liquefaciens.

2890-Eggs from a laboratory culture of the potato tuberworm were submitted by D. Brinkman, Albany, California, on April 1, 1969, for a pathogen check. Diagnosis: The eggs were contaminated with Bacillus thuringiensis var. thuringiensis.

2984 Several larvae and pupae of the potato tuberworm again were submitted by D. Brinkman on April 14, 1971. Diagnosis: Bacteriosis caused by Streptococcus sp., indicating a possible stress situation in the rearing conditions.

3028-Many laboratory-reared larvae were submitted by S. W. Broodryk, Pretoria, South Africa, on November 19, 
1971. Diagnosis: Bacteriosis caused by the potential pathogen Streptococcus faecalis var. liquefaciens.

\section{Phthorimaea sp.}

2595-Several dead specimens were submitted by L. Anderson, Palisade, Colorado, on April 8, 1966. Diagnosis: Bacteriosis: Streptococcus faecalis var. liquefaciens.

\section{Recurvaria milleri (Busch)}

2355-Seventeen specimens of the lodgepole needle miner from Yosemite National Park were submitted by G. R. Struble, Berkeley, California, on February 11, 1965. Diagnosis: Four of the specimens suffered from a granulosis virus. The remaining 13 showed no microbial etiology.

\section{Geometridae}

Abraxas grossulariata L.

2976-A few deteriorated larvae were received on December 21, 1970 from $\mathrm{T}$. Edlund, Vollebekk, Norway. Diagnosis: Virosis caused by a nuclear polyhedrosis virus.

\section{Alsophila pometaria (Harris)}

1885-One specimen was submitted by R. D. Frye, Fargo, North Dakota, on June 1, 1962. Diagnosis: Probably non-microbial.

1905-Another specimen was submitted by R. D. Frye on July 20, 1962. Diagnosis: Undetermined.

2261-Several specimens reared from eggs collected at Blue Ridge Parkway, Virginia, were submitted by A. T. Drooz, Asheville, North Carolina, on May 12, 1964. Diagnosis: Non-microbial.

\section{Anomis subulifera (GN)}

2665-Several larvae of the jute semilooper were submitted by A. Islam, Dacea, East Pakistan, on August 2, 1966. Diagnosis: Bacteriosis caused by the potential pathogen Bacillus cereus.

\section{Biston marginata Shiraki}

2954-Several diseased larvae of the black-headed looper were submitted by C. Chien $\mathrm{Hu}$, Lin-Kou Tea Experiment Station, Taiwan, on July 7, 1970. The larvae were collected from tea plants. Diagnosis: Mycoses caused by Aspergillus flavus, and A. ochraceous. Both were infective to Galleria larvae.

2964-Additional specimens of the black-headed looper collected from tea were received from C. Chien $\mathrm{Hu}$ on September 10, 1970. Diagnosis: Mycoses: Aspergillus sclerotium and a Penicillum sp.

\section{Buzura spp.}

1870-Several specimens were submitted by $\mathrm{K}$. W. Brown, Kampala, Uganda, on May 3, 1962. Diagnosis: Undetermined.

\section{Ennomos subsignarius (Hubner)}

2061 - Several laboratory-reared specimens were submitted by A. T. Drooz, Asheville, North Carolina, on June 3, 1963. Diagnosis: Probably nonmicrobial.

2071-One additional specimen was submitted by A. T. Drooz on June 14, 1963. It had been reared in an insectary and had died in the process of moulting after a delayed 4 th instar. Diagnosis: Non-microbial.

2255-Several more laboratoryreared specimens were submitted by $\mathrm{A}$. T. Drooz on April 22, 1964. Diagnosis: Mycosis caused by Paecilomyces farinosus.

2636-Ten larvae were received on June 21, 1966, from A. T. Drooz. Diagnosis: Several secondary, saprophytic fungi in the genus Penicillium.

2808-Four eggs of the elm spanworm were submitted by A. T. Drooz, Asheville, North Carolina, on February 19, 1968. Diagnosis: Non-microbial. 
Epirrita (Oporinia) autumnata Bkh.

2493-Some specimens were submitted by O. Tenow, Uppsala, Sweden, on October 12,1965 . They had been collected from the mountain birch (Betula pubescens ssp. tortuosa Led.) at the Tana River, Finmark, Norway, and at the River Jebrenjakk, Torne Lappmark, Sweden, on July 13 and August 8, 1965, respectively. The larvae would spin their hind extremities firmly to a twig, change body color to yellow-green, and become turgid; then they would bend backwards and bite their sides until the body wall was punctured. Diagnosis: Virosis: cytoplasmic polyhedrosis virus. A new record.

2526-Seven additional specimens of the larch looper from Forde, Sogn og Fjordane Fylke, Norway, were submitted by $\mathrm{O}$. Tenow, Uppsala, Sweden, on November 30, 1965. Diagnosis: Nonmicrobial.
Lambdina fiscellaria lugubrosa Guen.

2668-Several larvae were received from P. Shea, Berkeley, California, on August 8, 1966. Diagnosis: Virosis: nuclear polyhedrosis virus.

Unidentified Hemlock Looper, probably Lambdina sp.

2652- Two larvae were received from B. E. Wickman, Berkeley, California, on July 18, 1966. Diagnosis: Virosis: Nuclear polyhedrosis virus. (Fig. 17.)

\section{Nepytia near phantasmaria (Stkn.)}

2487-These specimens from Beartrap Creek near McCloud, California, were submitted by D. Dahlsten, Albany, California, on October 1, 1965. Diagnosis: Mycosis caused by Beauveria bassiana.

\section{Operophthera brumata $\mathrm{L}$.}

2494-Several larvae were submitted by 0 . Tenow, Uppsala, Sweden, on Oc-

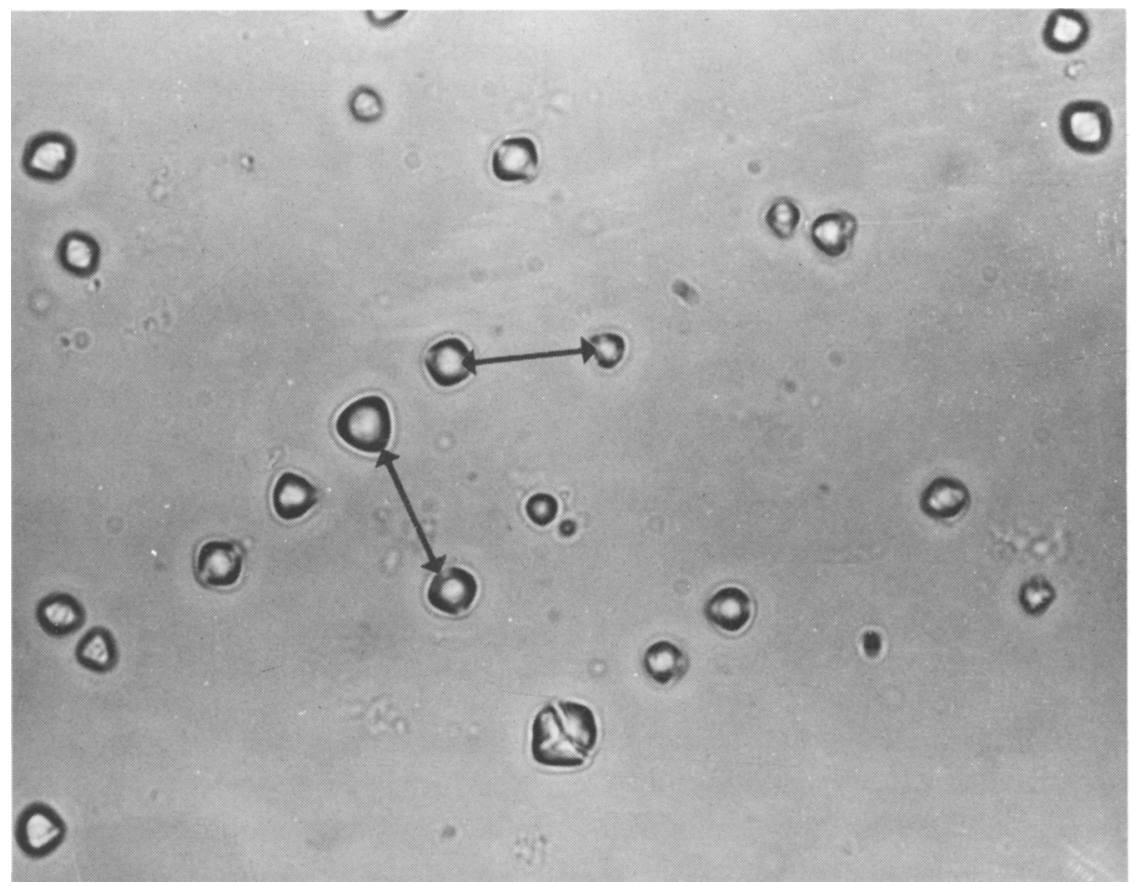

Fig. 17. Acc. No. 2652: Blood of Lambdina sp. containing polyhedra (arrows) of a nuclear polyhedrosis virus. Magnification $=2000 X$. 
tober 12,1965 . They had been collected at the River Jebrenjakk, Torne, Lappmark, Sweden, on August 8, 1965. Diagnosis: Virosis: cytoplasmic polyhedrosis virus.

\section{Paleacrita vernata (Peck)}

2996_-Three spring cankerworm larvae collected from Siberian elm in Bottineau, North Dakota, were submitted by J. Stein on June 21, 1971. Diagnosis: Non-microbial.

\section{Phaeoura mexicanaria (Grote)}

2967-Some larvae were submitted by C. E. Carlson, Missoula, Montana, on September 15, 1970. They were collected near Lame Deer, Montana. A bacterium was isolated by $\mathrm{K}$. Hughes (Corvallis, Oregon), who showed it to be pathogenic for larvae of this insect. Diagnosis: Bacteriosis caused by an unidentified gram negative bacterium.

\section{Semiothisa sexmaculata (Pack)}

2913-A few larvae were submitted on September 9, 1969 by J. Stein, Bottineau, North Dakota. The larvae were collected from larch trees at Denbigh, North Dakota, where about 1 per cent of the insect population was infected. Diagnosis: Virosis caused by a granulosis virus.

\section{Unidentified}

2240-Several specimens were collected in the Caroline Islands and submitted by F. A. Bianchi on March 11, 1964. Diagnosis: Virosis caused by a nuclear polyhedrosis virus.

2925-Eleven pupae collected from Pinus ponderosae near Ashland, Montana, were submitted on November 13, 1969 by J. R. Milodragovich, Missoula, Montana. Diagnosis: Mycosis caused by Paecilomyces farinosus.

3011-Several unidentified larvae of a geometrid collected from white fir, and then placed on an artificial medium were submitted by D. Dahlsten, Berkeley, California, on September 1, 1971. Diagnosis: Non-microbial.

\section{Hepialidae}

Oncopera mitocera (Turner)

2272-One larva was collected in Evelyn, Queensland, Australia, by J. H. D. Barnett, Brisbane, Australia, on June 12, 1964. Diagnosis: Mycosis caused by Fusarium sp.

\section{Oxycanus sp.}

1930-A mumified specimen of this subterranean caterpillar was received from G. A. H. Helson, Wellington, New Zealand, on August 15, 1962. The specimen was collected from pasture grass turf. Diagnosis: Mycosis caused by Metharrizium anisopliae. A new host record for this fungus.

\section{Oxycanus sp. (Walker)}

2337-These specimens were received from L. D. Crawford, Victoria, Australia, on November 6, 1964. This species is a pest of improved pastures. Diagnosis: Bacteriosis caused by $P$ seudomonas aeruginosa.

\section{Hesperidae}

2353-One unidentified hesperid larva was submitted by G. Rothschild, Sarawak, on January 25, 1965. Diagnosis: Mycosis caused by Spicaria rileyi.

\section{Hyponomeutidae}

Argyresthia cupressella Wlsm.

2623-One specimen collected in the Piedmont Pines District of Oakland, California, was submitted by L. Etzel on May 3, 1966. Diagnosis: Virosis caused by granulosis virus.

\section{Argyresthia sp.}

2351-Several larvae from Oakland, California, were submitted by C. S. Koehler, Berkeley, California, on January 25, 1965. Diagnosis: Probably nonmicrobial. 


\section{Incurrariidae}

Parategeticula pollinifera Davis

3008-J. Powell, Berkeley, California, submitted four dead larvae of the yucca moth that were dissected from yucca pods. Diagnosis: Non-microbial.

\section{Lasiocampidae}

\section{Dendrolimus spectabilis Butler}

2580-Several specimens of the pine caterpillar were submitted by Je Ho Ko, Seoul, Korea, on February 24, 1966. Diagnosis: Non-microbial. Only the secondary saprophytic fungi, Penicillium sp., Rhizopus nigricans, Fusarium sp., and a Dematiaceae were isolated.

\section{Gonometa sp., probably nysa Druce}

2488-Five larvae were submitted by K. W. Brown, Kampala, Uganda, on October 1, 1965. Diagnosis: Bacteriosis caused by Streptococcus faecalis.

\section{Malacosoma americanum (Fabricus)}

2045 - Several laboratory-reared specimens were submitted by A. D. Oliver, Baton Rouge, Louisiana, on April 27, 1963. Diagnosis: Virosis caused by a nuclear polyhedrosis virus.

\section{Malacosoma constricta Stretch}

2902-On July 11, 1969, C. L. Smola, San Francisco, California, submitted eggs and pupae of the Pacific ten caterpillar collected from oak at Jolon, California, on July 9, 1969. Diagnosis: Virosis caused by a nuclear polyhedrosis virus.

\section{Malacosoma disstria (Hubner)}

2257-Several specimens of the forest tent caterpillar were submitted by A. D. Oliver, Baton Rouge, Louisiana, on April 29, 1964. Diagnosis: Probable mycosis caused by Entomophthora sp.

2746-Some larvae and eggs were submitted by D. R. Kucera, Pineville, Louisiana, on April 18, 1967. Diagnosis: Larvae: virosis caused by a nuclear polyhedrosis virus. Eggs: either nonmicrobial, or if not, then the primary etiology had been obscured by secondary Penicillium sp.

2848-K. E. Evans, Twin Falls, Idaho, submitted several tent caterpillar larvae on July 17, 1968. These specimens were collected near Ririe, Jefferson County, Idaho, Diagnosis: Virosis caused by a nuclear polyhedrosis virus.

\section{Malacosoma sp.}

2408-Several specimens collected in Zion National Park on May 13, 1965, were submitted by W. H. Klein, Ogden, Utah, on May 18, 1965. Diagnosis: Bacteriosis caused by Bacillus thuringiensis var. thuringiensis.

2424-Some specimens were submitted by J. L. Frykman, Ogden, Utah, on June 25, 1965. Diagnosis: Virosis caused by a cytoplasmic polyhedrosis virus.

2435-Some specimens collected in Bryce Canyon National Park were submitted by M. McGregor, Ogden, Utah, on July 23, 1965. Diagnosis: Virosis caused by a cytoplasmic polyhedrosis virus.

2603-Several dead specimens were submitted by J. L. Frykman, Ogden, Utah, on April 20, 1966. They had been collected about 10 miles north of St. George, Utah, and were infected with a nuclear polyhedrosis virus. Diagnosis: Virosis caused by a nuclear polyhedrosis virus.

2816-Several larvae collected from bitterbrush in the Fish Lake National Forest, Utah, were submitted on April 1, 1968 by A. M. Rivas, Ogden, Utah. Diagnosis: Virosis caused by a nuclear polyhedrosis virus.

2819-Several larvae collected from chokecherry were received on April 8, 1968 from E. H. Juntunen, Missoula, Montana. Diagnosis: Virosis caused by a nuclear polyhedrosis virus. 


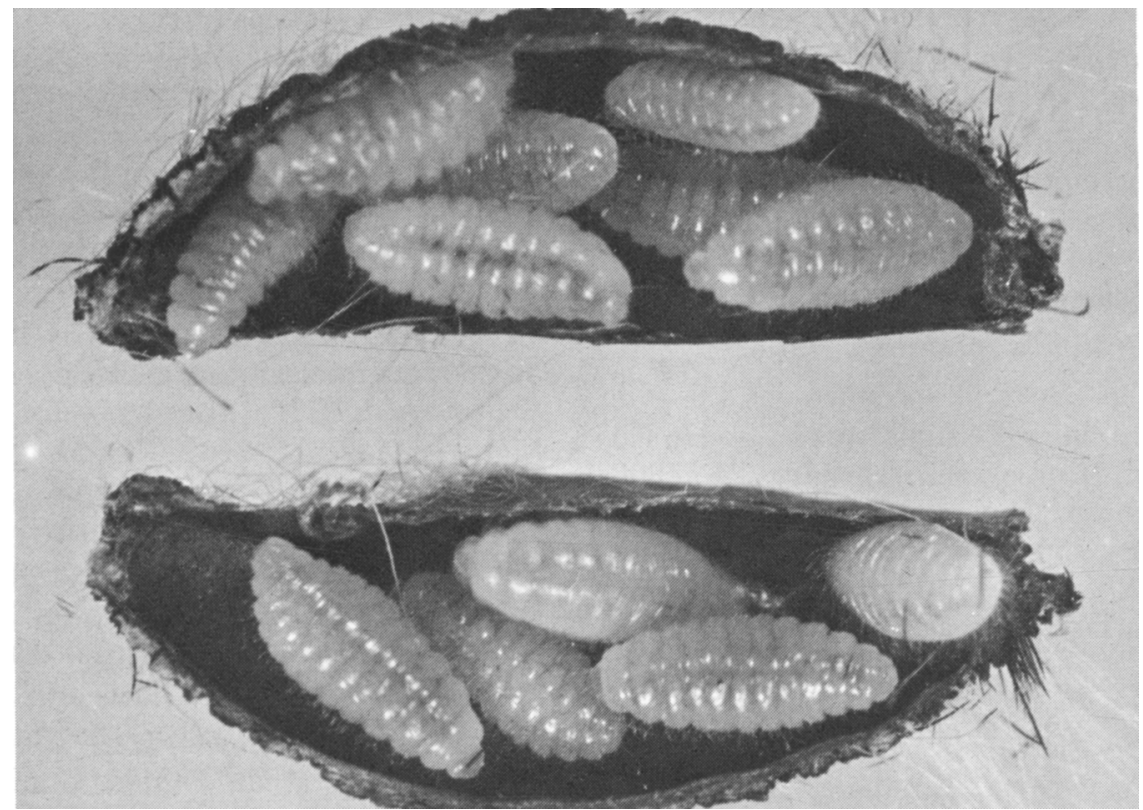

Fig. 18. Acc. No. 2002: Unidentified lasiocampid cocoon showing larvae of a hymenopterous parasite. Magnification $=10 \mathrm{X}$.

Trabala vishnu Lefebvre

2307-Three specimens were submitted on August 7, 1964 by O. S. Bindra, Jabalpur, India. No collection data or related information was supplied. Diagnosis: Non-microbial.

\section{Unidentified}

2002_Several laboratory reared specimens were submitted by I. M. Din, Rawalpindi, Pakistan, on January 18, 1963. Diagnosis: Parasitism caused by a hymenopterous parasite. (Fig. 18.)

\section{Limacodidae}

Darna trima (Moore)

2057-On May 20, 1963, thirteen larvae were received from B. J. Wood, Johore, Malaya. Diagnosis: Virosis caused by granulosis virus. New record. (Fig. 19.)

2337-Additional specimens were submitted by B. J. Wood on March 5, 1964. The disease appeared to be epizootic. Diagnosis: Virosis caused by a granulosis virus.
2840-Many dried larvae collected in the northern part of Borneo were received from B. J. Wood, Johore, Malaysia, on June 18, 1968. Diagnosis: Possible bacteriosis caused by the potential pathogen Bacillus cereus.

\section{Parasa lepida (Constantini)}

2242_-These field-collected specimens were submitted by U. B. M. Ekanayake, Ceylon, on February 27, 1964. Mr. Ekanayake reported that they appeared sluggish. Diagnosis: Bacteriosis: Streptococcus sp.

\section{Parasa sp.}

1865-Several hundred specimens were submitted by J. J. H. Szent-Ivany, Konedobu, Papua, on April 18, 1962. Diagnosis: Probable parasitism with secondary saprophytic invaders.

\section{Sibine apicalis Dyar}

2568-Two dead larvae were submitted by C. S. Stephens, Changuinola, Panama, on February 14, 1966. Diagno- 


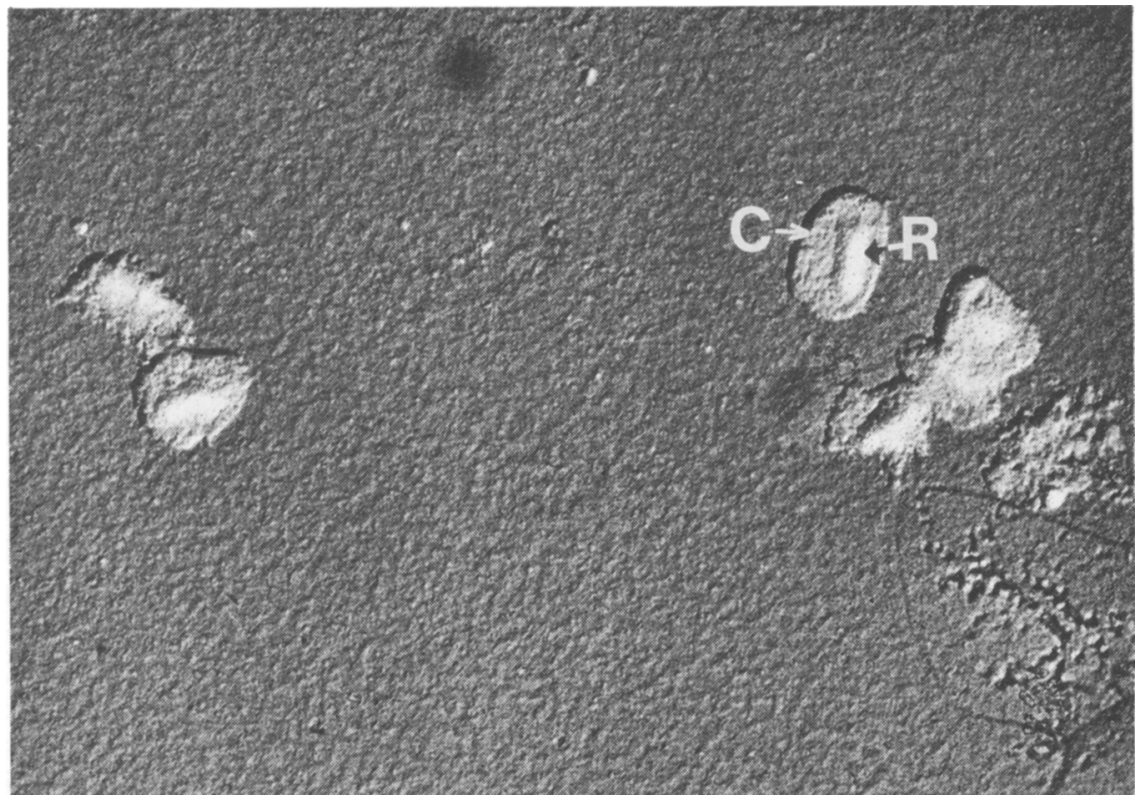

Fig. 19. Acc. No. 2057: Electron micrograph of a granulosis virus from Darna trima, with virus rods $(R)$ in partially dissolved capsules $(C)$. Magnification $=34,000 \mathrm{X}$.

sis: Bacterioses caused by Streptococcus faecalis and Proteus sp.

2578-One dead specimen was submitted by C. S. Stephens, Changuinola, Panama, on March 2, 1966. The insects were reported to be abundant and dying in large numbers. Diagnosis: Bacterioses caused by Serratia marcescens and Streptococcus faecalis.

2951--Several diseased larvae from banana leaves in Panama were submitted by C. S. Stephens, Changuinola, Panama, on June 2, 1970. Diagnosis: Mycosis caused by Beauveria tenella.

\section{Sibine stimulea (Clements)}

1895-Several specimens were submitted by D. McKay, St. Petersburg, Florida, on July 3, 1962. Diagnosis: Probably non-microbial.

\section{Sibine sp.}

2579-One specimen was submitted by C. S. Stephens, Changuinola, Panama. The insects were reported to be abundant and dying in large numbers. Diagnosis: Bacteriosis: Streptococcus faecalis.

\section{Lymantriidae}

\section{Dasychira plagiata (Walker)}

1873-Over 50 larvae of the pine tussock moth were submitted by J. Childs, St. Paul, Minnesota, on May 10, 1962. Diagnosis: Virosis caused by a nuclear polyhedrosis virus. The first record of a virus infection of this insect in the Americas. (Fig. 20.)

2474_Several specimens were submitted by R. V. Gorsuch, Missoula, Montana, on September 21, 1965. Diagnosis: Virosis caused by a nuclear polyhedrosis virus.

2704-Some larvae were received from R. L. Lyon, Berkeley, California, on November 16, 1966. Diagnosis: Virosis, caused by a nuclear polyhedrosis virus.

2761-Two larvae were submitted by E. H. Juntunen, Missoula, Montana, on July 21, 1967. Diagnosis: Mycosis; how- 


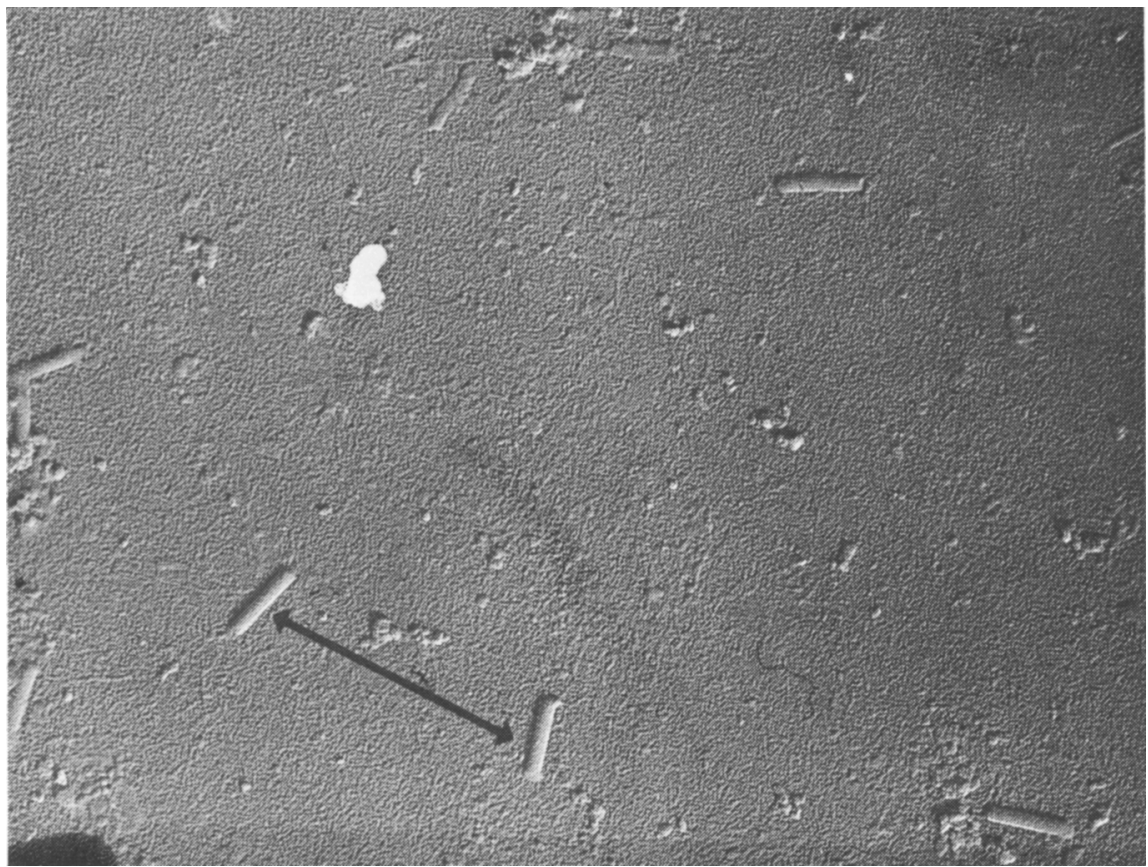

Fig. 20. Acc. No. 1873: Electron micrograph of nuclear polyhedrosis virus rods (arrows) from Dasychira plagiata. Magnification $=22,500 \mathrm{X}$.

ever, the fungus was no longer viable and could not be identified. Penicillium sp. and Aspergillus niger, both saprophytes, were also isolated.

\section{Euproctis lunata Walker}

2612-Several specimens were submitted by R. B. Subba Rao, New Delhi, India, on May 10, 1966. The material was divided into two lots, each suffering from a different disease. Diagnoses: 1 . Mycosis caused by an Entomophthora sp. 2. Bacteriosis caused by Bacillus cereus.

\section{Hemerocampa vetusta (Boisduval)}

2329-D. Dahlsten, Albany, California, submitted two dead specimens on October 13, 1964, which had been collected on white fir near Canyon Creek road, Modoc County, California. Diagnosis: Virosis caused by a nuclear polyhedrosis virus.
Orgiya pseudotsugata (McDunnough)

2094-Several specimens of the Douglas fir tussock moth were submitted by H. C. Roffler, Missoula, Montana, on August 5, 1963. The disease appeared to be an epizootic. Diagnosis: Nuclear polyhedrosis virus.

2370-Several larvae from the field that had been laboratory-reared were submitted by D. Wood, Berkeley, California, on March 22, 1965. Diagnosis: Virosis caused by a nuclear polyhedrosis virus.

2419, 2423, 2429, 2432, 2433-Some larvae were submitted by D. Dahlsten, Albany, California, on June 15, June 23, July 2, July 19, July 21, 1965. Egg masses were collected from various locations throughout California and reared on an artificial diet. Diagnosis: Virosis caused by a nuclear polyhedrosis virus. (Dahlsten and Thomas, 1969.) 
2456-Additional specimens collected at various locations in Califormia and reared in the laboratory by $\mathrm{D}$. Dahlsten were submitted on August 10, 1965. Diagnosis: Non-microbial.

2486_-Specimens similar to the above were again submitted by D. Dahlsten on October 1, 1965. Diagnosis: General bacterial deterioration-no primary microbial etiology evident.

2600-Numerous dead larvae were again submitted by $\mathrm{D}$. Dahlsten on April 19, 1966. They had been collected at Goose Lake, 10 miles west of New Pine Creek, Modoc County, California, as egg masses and reared in the laboratory on artificial media. Diagnosis: Virosis, caused by a nuclear polyhedrosis virus. (Dahlsten and Thomas, 1969.)

2609 - Several laboratory-reared specimens were submitted by D. Wood, Berkeley, California, on May 5, 1966. Diagnosis: Virosis, caused by a nuclear polyhedrosis virus.

2639-Several more larvae reared in the laboratory from field-collected eggs were submitted by D. Dahlsten, Albany, California, on June 23, 1966. Diagnosis: Virosis, caused by a nuclear polyhedrosis virus. (Dahlsten and Thomas, 1969.)

2659-Several larvae were received from D. Wood, California, on July 27, 1966. Diagnosis: Non-microbial.

2667-A few larvae were submitted by $\mathrm{P}$. Shea, Berkeley, California, on August 8, 1966. Diagnosis: Virosis, caused by a nuclear polyhedrosis virus.

2677-Additional larvae were received from D. Dahlsten on August 28, 1966. Diagnosis: Virosis, caused by a nuclear polyhedrosis virus. (Dahlsten and Thomas, 1969.)

2687-2694-A number of eggs, larvae, and adults of the Douglas fir tussock moth were submitted by D. Wood, Berkeley, California, from September 20 through September 26, 1966. Diagnoses: 2687 (adults) = Bacillus cereus; 2688 (eggs) = non-microbial; 2689 (lar- vae $)=$ non-microbial $; 2690$ (larvae) $=$ non-microbial; 2691 (adult) = non-microbial $; 2692$ (larvae) $=$ Bacillus cereus var. mycoides; 2693 (adults) $=$ B. cereus; 2694 (adults) $=$ B. cereus.

2713-Some dead adults were received from A. Cameron, Berkeley, California, on December 21, 1966. Diagnosis: Bacteriosis caused by Bacillus thuringiensis var. thuringiensis.

2717-Several larvae were received from A. Cameron on January 5, 1967. Diagnosis: Non-microbial.

3015 - Many field-collected larvae collected near Nevada Point, Nevada County, California, were submitted by L. E. Browne, Berkeley, California, on September 15, 1971. Diagnosis: Nonmicrobial. It is possible that death was due to a severe heat wave since many oak worms had died of heat exhaustion during the same period.

3017-Several additional larvae were submitted by D. Dahlsten on September 24,1971 . These were also collected near Nevada Point, California. Diagnosis: Virosis caused by a nuclear polyhedrosis virus.

\section{Orgyia turbata Butler}

1997-B. Meksongsee, Bangkok, Thailand, submitted specimens on December 27, 1962. Diagnosis: Polyhedrosis virus. A new record for this host. (Fig. 21.)

\section{Noctuidae}

Achaea janata (L.)

1938-Laboratory-reared larvae of the castor bean looper were submitted by B. Meksongsee, Bangkok, Thailand, on September 4, 1962. The submitted specimens had been inoculated with a suspected pathogen by oral feeding and were submitted with the request for identification of the pathogen. Diagnosis: Virosis caused by a granulosis virus. (Fig. 22.) 


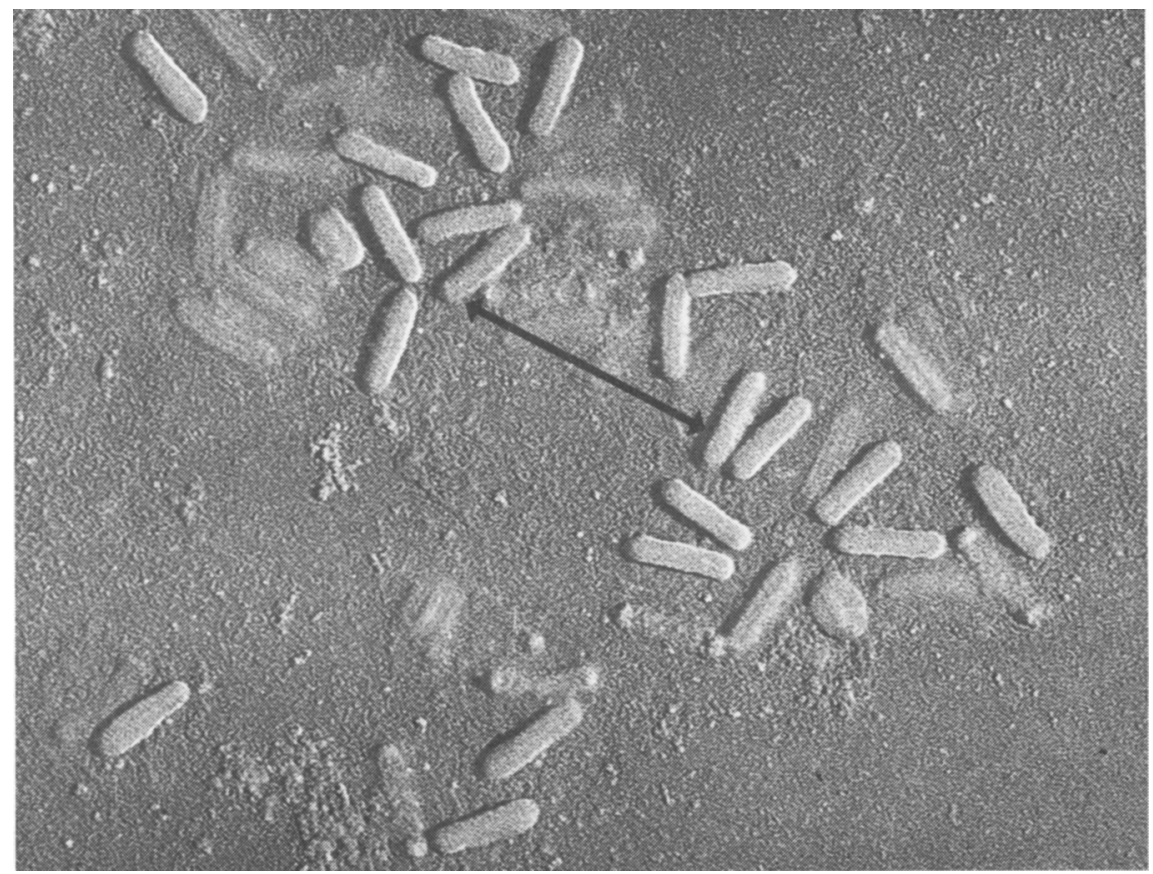

Fig. 21. Acc. No. 1997 : Electron micrograph of nuclear polyhedrosis virus rods (arrows) from Orgyia turbata. Magnification $=29,000 \mathrm{X}$.

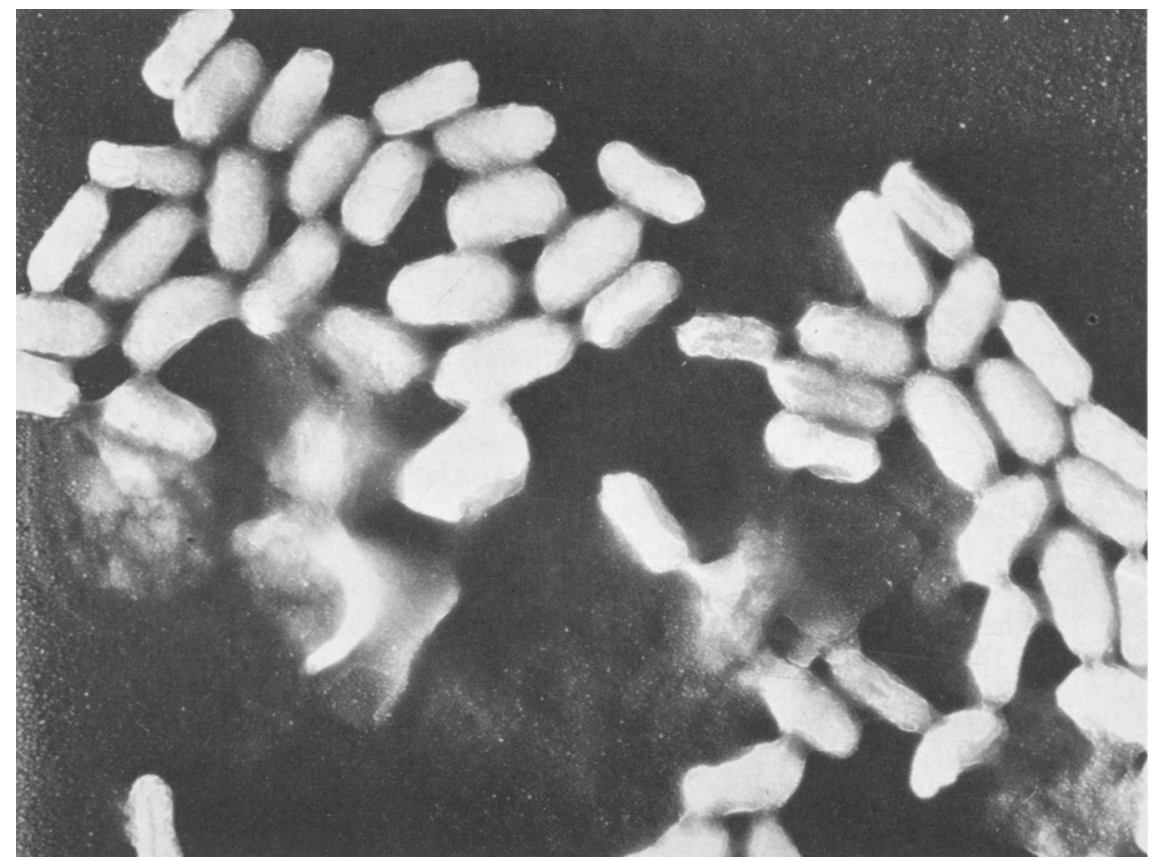

Fig. 22. Acc. No. 1938: Electron micrograph of granulosis virus capsules from Achaea janata. Magnification $=26,500 \mathrm{X}$. 
2364-Some larvae were received from J. W. Beardsley, Honolulu, Hawaii, on March 11, 1965. Diagnosis: Bacteriosis caused by Bacillus cereus var. mycoides.

\section{Alabama argillacea (Hübner)}

2076 - Several laboratory-reared specimens of the cotton leafworm were submitted by H. Alcaraz, Buga, Valle, Colombia, on June 26, 1963. Diagnosis: microsporidiosis caused by Nosema sp.

2289 and 2290_-On July 6, 1964, specimens of the cotton leafworm were submitted by R. C. Hunter, Bogota, Colombia. The specimens under accession 2289 were collected at Dorado (Tolima), Colombia, and those under No. 2290 were collected at Palmira (Valle), Colombia. The fungus was identified directly from the specimens, and from cultures on artificial media. Diagnosis: Mycosis: Spicaria rileyi.

2291 and 2292-Additional dead larvae were received on July 6, 1964, from R. C. Hunter, Bogota, Colombia. The specimens were deteriorated and putrefied. Diagnosis: Non-microbial. Possible insecticide poisoning.

2297-Several putrid specimens were received on July 10, 1964, from S. Shima K., Jr., Palmira, Valle, Colombia. Diagnosis: Microsporidiosis: Nosema sp., and secondary bacteriosis.

2992_On May 27, 1971, three dead larvae of the cotton leafworm were received from L. Falcon, Maragua, Nicaragua. Diagnosis: Mycoses in two larvae, caused by a Penicillium sp., and Spicaria rileyi. The cause of death in the third larva was evidently non-microbial.

\section{Agrotis orthogonia (Morrison)}

2227 - Several laboratory-reared specimens were submitted by L. A. Jacobson, Lethbridge, Alberta, Canada, on February 4, 1964. Diagnosis: Non-microbial.
Agrotis ypsilon (Von Rottenburgh)

1896_-One specimen was collected and submitted by M. D. De Silva, Gurutalawa, Ceylon, on July 16, 1962. Diagnosis: Undetermined.

\section{Anticarsia gemmatalis (Hübner)}

2211-Several specimens of the velvetbean caterpillar were submitted by R. C. Hunter, Palmira, Colombia, on December 16, 1963. Diagnosis: Mycosis caused by Spicaria rileyi.

2287-On July 6, 1964, additional specimens were received from $R$. C. Hunter, Palmira, Colombia. These were collected from a population at Bolo, Colombia, where nearly 100 per cent of the larvae had died of a fungus infection. Diagnosis: Mycosis: Spicaria rileyi.

2332_Several larvae were submitted by L. C. Kuitert, Gainesville, Florida, on October 26, 1964. The specimens were infected with a fungus and covered with mycelium and light green spores. Diagnosis: Mycosis caused by Spicaria rileyi.

\section{Calophasia lunula (Husn.)}

2695-Several larvae were received from R. Hawkes, Albany, California, on October 5, 1966. Diagnosis: Bacteriosis: Streptococcus infection.

2931-L. Andres, Albany, California, submitted some laboratory reared larvae on December 19, 1969. Diagnosis: Non-microbial.

\section{Chorizagrotis agrestis (Grote)}

2833-Several larvae of the western armyworm were received from $\mathrm{K}$. $\mathrm{E}$. Evans, Twin Falls, Idaho, on May 24, 1968. The larvae had been collected from crested wheat grass north of Kimama, Blaine County, Idaho. Diagnosis: Non-microbial.

\section{Eligma narcissus (Cramer)}

2037-Agar slants of fungus cultures isolated from pupae were submitted 


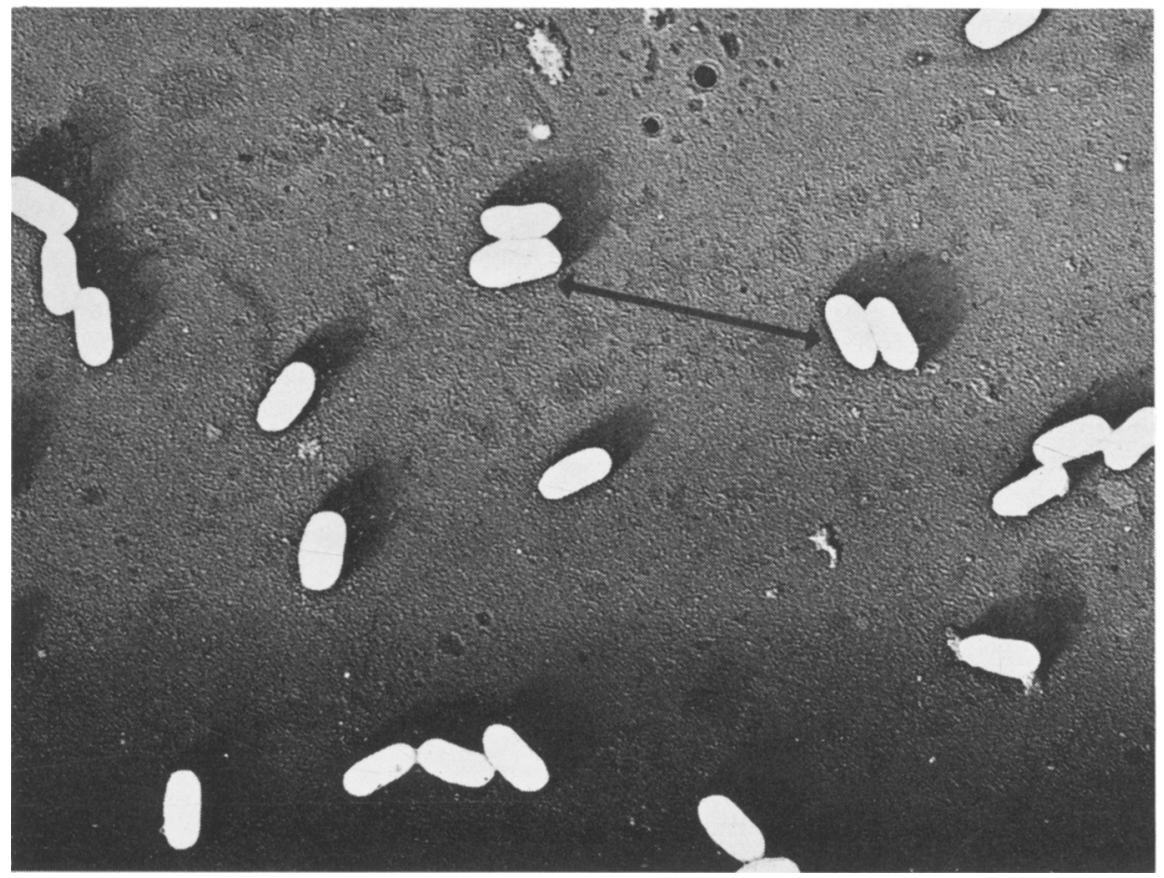

Fig. 23. Acc. No. 2243: Electron micrograph of granulosis virus capsules (arrows) from Eupsilis satelitia. Magnification $=21,250 \mathrm{X}$.

by J. C. Basu Choudhuri, Coimbatore, India, on April 1, 1963. Identification: Beauveria bassiana.

\section{Eupsilis satelitia (L.)}

2243-Several specimens were submitted by G. Taksdal, Vollebekk, Norway, on March 23, 1964. Diagnosis: Virosis caused by a granulosis virus. New record. (Fig. 23.)

\section{Euxoa ochrogaster (Guenee)}

2284-Several larvae were received from R. H. Washburn, Palmer, Alaska, on July 3, 1964. The larvae were collected in the field after an outbreak. Diagnosis: Bacteriosis: non-chromogenic strain of Serratia marcescens.

\section{Heliothis armigera (Hübner)}

2564-Six dead specimens were submitted by R. C. Patel, Anand, Gujarat, India, on February 10, 1966. Diagnosis:
Virosis caused by a nuclear polyhedrosis virus.

\section{Heliothis philoxiphaga (Grote and Robinson)}

2387-Some laboratory reared specimens were submitted by L. A. Falcon, Berkeley, California, on April 9, 1965. Diagnosis: Bacteriosis caused by Pseudomonas aeruginosa.

\section{Heliothis virescens (Fabricius)}

2295-Six specimens of the tobacco budworm were received from S. Shima K., Jr., Palmira, Valle, Colombia, on July 10, 1964. The cadavers were completely colonized by a fungus and covered with light green spores. Diagnosis: Mycosis-Spicaria rileyi.

2308B-Some laboratory reared specimens were submitted by R. C. Hunter, Bogota, Colombia, on August 25, 1964. Diagnosis: Virosis caused by a nuclear polyhedrosis virus. 
2315-Several specimens which had deteriorated to a fluid mass were received on September 10, 1964, from $R$. C. Hunter, Palmira, Valle, Colombia. Diagnosis: Virosis caused by a nuclear polyhedrosis virus.

\section{Heliothis zea (Boddie)}

1860-Several specimens were submitted by E. C. Klostermeyer, Prosser, Washington, on April 11, 1962. Diagnosis: Mycosis caused by Beauveria bassiana.

2469-One specimen was submitted by W. Kane, Berkeley, California, on August 31, 1964. Diagnosis: Bacteriosis caused by Serratia marcescens.

2504 -Specimens were submitted by L. Etzel, Berkeley, California, on October 27, 1965. They had been field-collected and reared on artificial medium in the laboratory. Diagnosis: External fungus only.

2516-One dead specimen was submitted by L. Etzel, Berkeley, California, on November 22, 1965, with a request for the identification of a fungus growing on the cadaver. Diagnosis: Saprophytic fungus, Penicillium sp.

2684 -Several larvae were received from D. G. Bottrell, Stillwater, Oklahoma, on Sepember 19, 1966. Diagnosis: A: A1 and A2-non-microbial; B: B1 and B2-bacteriosis, strain of Streptococcus faecalis; C: non-microbial; D: virosis, granulosis virus; E: no apparent microbial etiology.

2838-On June 7, 1968, several larvae and pupae of the corn earworm were received from laboratory cultures of J. J. Ellington, Las Cruces, New Mexico, for a pathogen check. Diagnosis: Non-microbial.

2885-On February 17, 1969, fourteen additional larvae were submitted by J. J. Ellington for a pathogen check. Diagnosis: Virosis caused by a nuclear polyhedrosis virus.
2910-P. Velez, Davis, California, submitted several deteriorated larvae on August 27, 1969. Diagnosis: Probably non-microbial. The submitted specimens had been well deteriorated by the action of secondary bacteria. If a primary microbial etiology was present, it had been masked by the action of secondary saprophytes.

2911-Additional larvae were submitted by $\mathrm{P}$. Velez on September 4, 1969. The larvae were flaccid and in an advanced state of deterioration. Diagnosis: Non-microbial.

2919-Larvae of the corn earworm collected from various locations in Mexico were submitted by L. A. Falcon, Berkeley, California, on October 1, 1969. Diagnosis: Nuclear polyhedrosis virus present in all lots.

2982-Four larvae were received from G. Leon Quant, Leon, Nicaragua, on February 3, 1971. The larvae were field-collected and reared in the laboratory. Diagnosis: Mycoses caused by $A s$ pergillus flavus and Spicaria rileyi.

2993-L. Falcon submitted three dead larvae from Managua, Nicaragua, on May 27, 1971. Diagnosis: Microsporidiosis in one specimen, probably caused by a Nosema sp. Mycosis in another specimen caused by Spicaria rileyi. The cause of death in the third specimen was probably non-microbial.

\section{Heliothis sp.}

2133-These specimens, which were field-collected and reared in the laboratory, were submitted by $\mathrm{H}$. Alcaraz, Buga, Valle, Colombia, on August 20, 1963. Insecticides had been applied to the area. Diagnosis: Mycosis caused by Spicaria rileyi.

2294-On July 10, 1964, completely deteriorated specimens of Heliothis sp. were received from S. Shima K., Jr., Palmira, Colombia. Diagnosis: Virosis: nuclear polyhedrosis virus. 


\section{Heliothis sp. (zea or philoxiphaga)}

2420 -Some specimens were submitted by L. Ruud, Kerman, California, on June 17, 1965. They had been reared on a semi-synthetic diet in the laboratory. Diagnosis: Some showed no microbial etiology; others had a secondary bacteriosis caused by Streptococcus sp.

\section{Laphygma exempta (Walker)}

2271-These specimens were submitted by J. H. D. Barnett, Brisbane, Queensland, Australia, on June 12, 1964. Diagnosis: Mycoses caused by Entomophthora sphaerosperma and Spicaria sp.

\section{Laphygma exigua (Hübner)}

2040-Slides of nematodes taken from the beet armyworm were submitted by T. V. Venkatraman, Khartoum North, Sudan, on April 11, 1963. Diagnosis: Panagrolaimus sp.

\section{Laphygma frugiperda (J. E. Smith)}

2000-Some specimens were submitted by R. C. Hunter, Buga, Valle, Colombia, on January 18, 1962. Diagnoses: Bacteriosis caused by Aerobacter aerogenes; Mycoses caused by Aspergillus flavus and Spicaria rileyi.

2134-Additional specimens were submitted by R. C. Hunter, Valle, Colombia, on August 20, 1963. The area had been treated with insecticides. Diagnosis: Mycosis caused by Spicaria rileyi.

2241-Several specimens were received from I. S. de Crouzel, Buenos Aires, Argentina, on March 16, 1964. These specimens were reared in the laboratory from eggs collected in the field. Diagnosis: Probable bacteriosis caused by Streptococcus spp. with secondary saprophytic fungus, Masoniella sp. and a fortuitous bacterium, Erwina sp.

2246-Additional specimens were submitted by I. S. de Crouzel, Buenos
Aires, Argentina, on March 26, 1964. Diagnosis: Non-microbial.

2288-One specimen of the fall armyworm was submitted by R. C. Hunter, Bogota, Colombia, on July 6, 1964. The specimen was completely colonized by a fungus. Diagnosis: Mycosis: Spicaria rileyi.

2407-Some specimens were submitted by A. Silveira-Guido, Montevideo, Uruguay, on May 17, 1965. Diagnosis: Bacterioses caused by Serratia marcescens and Pseudomonas aeruginosa.

Leucania latiuscula (Herrick-Schafter)

2347-Several larvae were submitted by W. G. Genug, Belle Glade, Florida, on January 11, 1965. The cadavers were completely colonized by a fungus. Diagnosis: Mycosis: Spicaria rileyi.

\section{Mythimna sp.}

3030-Many field-collected larvae of this noctuid were submitted by A. J. M. Carnegie, Mount Edgecomb, South Africa, on November 19, 1971. Diagnosis: Mycosis caused by an Entomophthora $\mathrm{sp}$.

Papaipema pupurifascia (Grote and Robinson)

2450-These specimens were submitted by R. Anderson, Madison, Wisconsin, on August 4, 1965. Diagnoses: Mycosis caused by Beauveria bassiana in some of the specimens, by Hirsutella sp. in others. Some showed a granulosis virus, while others showed a bacteriosis caused by Streptococcus faecalis.

2460-Additional larvae were submitted on artificial media by R. J. Anderson, Madison, Wisconsin, on August 23, 1965. Diagnosis: Bacterioses caused by Bacillus cereus, Proteus sp., Aerobacter grp. C, and Pseudomonas aeruginosa. 


\section{Peridroma margaritosa (Haworth)}

2363-These cutworm larvae were received from J. W. Beardsley, Honolulu, Hawaii, on March 11, 1965. Diagnosis: Virosis caused by a nuclear polyhedrosis virus.

2385-Specimens were collected at the Kahua Ranch, Waimea-kohala, Hawaii, on March 20 to April 1, 1965, by Y. Tanada, Berkeley, California. Diagnoses: Bacteriosis caused by $\mathrm{Pseu}$ domonas aeruginosa and another unidentified bacterium; mycosis caused by Beauveria bassiana; non-microbial in others.

2395-Some specimens were submitted by W. C. Mitchell, Honolulu, Hawaii, on April 26, 1965. Diagnosis: Bacteriosis caused by Bacillus cereus.

\section{Peridroma sp.}

2399_Some specimens were collected in Kamuela, Hawaii, on April 14-20, 1965, by Y. Tanada, Berkeley, California. Diagnosis: Bacterioses caused by Bacillus cereus, B. cereus var. $m y$ coides and Pseudomonas aeruginosa.

2404-Additional specimens were collected in Hawaii and submitted by Y. Tanada, Berkeley, California, on May 12, 1965. Diagnoses: Mycoses caused by Entomophthora sp. and Beauveria bassiana; bacteriosis caused by Streptococcus sp., Bacillus cereus var. mycoides, and Bacillus cereus; virosis caused by a polyhedrosis virus.

\section{Persectania aversa (Walker)}

2027-One larva was submitted by G. A. H. Helson, Wellington, New Zealand, on March 18, 1963. Diagnosis: Mycosis caused by Metarrhizium anisopliae.

2085-Several more specimens were submitted by G. A. H. Helson, Wellington, New Zealand, on July 18, 1963. Diagnosis: Mycosis caused by Entomophthora sp.
Prodenia androgea (Creamer)

2360_These specimens from La Estrella Valley, Costa Rica, were submitted by F. Lara E., Limon, Costa Rica, on February 19, 1965. Diagnosis: Virosis caused by a granulosis virus. New record.

2445-Additional laboratory reared specimens were submitted by F. Lara E., Limon, Costa Rica, on July 28, 1965. Diagnosis: Primary etiology was obscured by the action of acarid mites.

\section{Prodenia litura (Fabricus)}

2097 - Several laboratory-reared specimens were submitted by U. Ekanayake, Lunuwila, Ceylon, on August 9, 1963. Diagnosis: Micrasporidiosis caused by a Nosema sp. New record.

2217-Eight insectary-reared specimens were submitted by U. Ekanayake, Lunuwila, Ceylon, on December 31, 1963. Diagnosis: Mycosis caused by Aspergillus flavus.

2268, 2269 - Additional laboratoryreared specimens were submitted by U. Ekanayake, Lunuwila, Ceylon, on June 5 and June 11, 1964. Diagnosis: Microsporidiosis caused by Nosema sp.

2398-Some specimens were submitted by S. Rao, Kuala Lumpur, Malaysia, on April 27, 1965. The area had been treated with endrin. Diagnosis: Bacterioses caused by Streptococcus sp. near faecalis and Aerobacter liquifaciens.

2832-M. M. Altahtawy, Alexandria, Egypt, submitted several dead larvae collected from cotton on April 24, 1968. Diagnosis: Virosis caused by a nuclear polyhedrosis virus.

2854 Two bacterial cultures isolated from $P$. litura by M. M. Altahtawy were received on August 12, 1968, with a request for their identification. Diagnosis: The bacteria were identified as Bacillus cereus and an Aerobacter sp. 
2933-Four dead larvae were received from O. S. Bindra, Ludhiana, India, on February 10, 1970. Diagnoses: Two specimens suffered a mycosis caused by Aspergillus flavus; one suffered a bacteriosis caused by the potential pathogen Streptococcus faecalis; and the fourth suffered a virosis caused by a granulosis virus.

\section{Prodenia ornithogalli (Guenee)}

2149_-Several specimens were submitted by G. T. Okamura, Sacramento, California, on September 3, 1963. This disease appeared to be epizootic. Diagnosis: Nuclear polyhedrosis virus.

2592-Several dead specimens were submitted by D. G. Bottrell, Stillwater, Oklahoma, on March 29, 1966. Diagnosis: Bacteriosis caused by a Streptococcus $\mathrm{sp}$.

2685-Three additional larvae were received from D. G. Bottrell, Stillwater, Oklahoma, on September 19, 1966. Diagnosis: 1. Bacteriosis, strain of Streptococcus faecalis. 2. Mycosis with secondary bacteriosis. Attempts to isolate and identify the fungus were unsuccessful.

\section{Prodenia sp.}

2001-These specimens were submitted by H. Alcaraz, Buga, Valle, Colombia, on January 18, 1963. Diagnosis: Mycosis caused by Spicaria rileyi and bacterioses caused by Aerobacter aerogenes, and Streptococcus sp.

2077-These specimens were submitted by R. C. Hunter, Buga, Valle, Colombia, on June 26, 1963. Lead arsenate had been applied to the field before collection. Diagnosis: Undetermined, probably non-microbial.

2195_-Six specimens were submitted by F. A. Estrada Rizo, Managua, Nicaragua, on November 14, 1963. They had been collected in Los Laureles, where the disease appeared to have reached epizootic proportions. Diag- nosis: Mycosis caused by Spicaria rileyi.

2953-Four dead larvae were submitted by H. Eugene Ostmark, La Lime, Honduras, on June 30, 1970. The larvae were collected from peanuts in Rivas, Nicaragua, and had been fed leaves coated with the ground bodies of other larvae which Mr. Ostmark suspected had been killed by a virus. Diagnosis: Virosis caused by a nuclear polyhedrosis virus.

\section{Protolevcania albilinia (Hübner)}

2631-Three larvae were submitted by E. A. Wood, Stillwater, Oklahoma, on June 3, 1966. Diagnosis: Specimens 1 and 2 showed secondary bacteriosis and mycosis. Specimen 3 was parasitized by a chalcid parasite. It is possible that 1 and 2 were also parasitized.

\section{Pseudoplusia includens (Walker)}

2865-Several larvae collected from soybean were submitted by L. D. Newsom, Baton Rouge, Louisiana, on October 7, 1968. Diagnosis: Bacteriosis caused by Bacillus finitimus, with associated secondary infection caused by Streptococcus faecalis and $\boldsymbol{S}$. faecalis var. liquefaciens.

2901-One larva of this semi-looper collected near Belle Glade, Florida, was received from M. J. Janes on July 3, 1969. Diagnosis: Mycosis caused by an Entomophthora sp.

\section{Pseudaletia separata}

2064 - This specimen was submitted by G. A. H. Helson, Wellington, New Zealand, on June 5, 1963. Diagnosis: Mycosis caused by Entomophthora sp.

\section{Pseudaletia unipuncta (Haworth)}

1955-Several armyworms were submitted by R. D. Frye, Fargo, North Dakota, on September 25, 1962. Diagnosis: Non-microbial.

2026-Eleven larvae and two pupae 
were submitted on March 13, 1963, by I. de Crouzel, Buenos Aires, Argentina. The specimens were collected from a natural population suffering a disease epizootic. Diagnosis: Viroses caused by a granulosis virus and a nuclear polyhedrosis virus.

2198-Some specimens were submitted by C. S. Morey and E. M. Caseus, Montevideo, Uruguay, on November 19, 1963. Diagnosis: Mycosis caused by Entomophthora sphaerosperma.

2343-Eight dead specimens were submitted with no background information, by Silveira-Guido, Montevideo, Uruguay. The specimens were collected in Rincon del Pino, Uruguay. Diagnosis: Non-microbial.

2557-One dead specimen was submitted by M. Zahurul Alem, Dacca, East Pakistan, on January 5, 1966. Diagnosis: Bacillus cereus and Streptococcus faecalis var. liquefaciens.

\section{Pseudaletia sp.}

2401-One larva was submitted by M. Z. Alam, Dacea, East Pakistan, on May 10, 1965. Diagnosis: Bacterioses caused by Bacillus cereus and Pseudomonas aeruginosa.

\section{Sacadodes pyralis (Dyar)}

2154 - Several laboratory-reared specimens were submitted by R. C. Hunter, Buga, Valle, Colombia, on September 5, 1963. Diagnosis: Nonmicrobial.

\section{Sesamia cretica (Lededrer)}

2361-H. N. Plaut, Haifa, Israel, submitted several larvae on March 3, 1965. Diagnosis: Virosis caused by a granulosis virus.

2366-Additional specimens were submitted by $H$. N. Plaut, Haifa, Israel, on March 15, 1965. Four fungi were isolated from the cadavers, but showed negative results on infectivity tests. Diagnosis: Undetermined.
Spodoptera exempta (Walker)

2194-Several specimens and two vials of a virus suspension from a laboratory colony of this insect were submitted by E. S. Brown, Kikuyu, Kenya, on November 9, 1963. Diagnosis: Virosis caused by a nuclear polyhedrosis virus.

2300_-Several larvae from a laboratory culture were received from $G$. Swaine, Arusha, Tanganyika, on July 14, 1964. Diagnosis: Probable bacteriosis caused by Streptococcus spp.

\section{Spodoptera exigua (Hübner)}

2666-Several larvae were received from A. Islam, Dacca, East Pakistan, on August 2, 1966. Diagnosis: Virosis caused by a nuclear polyhedrosis virus.

3013-Many dead larvae were submitted by $\mathrm{K}$. Eveleens, Albany, California, on September 13, 1971. The specimens were collected from cotton in an area where many larvae had died of a nuclear polyhedrosis virus. Submitted larvae developed slowly, failed to mature, and gradually died. Diagnosis: Bacteriosis caused by the potential pathogen Streptococcus faecalis. A sudden heat wave may be the stress factor that promoted infection by this bacterium.

Spodoptera frugiperda (Abbot and Smith)

3036-Many last instar larvae of this armyworm collected from corn in Palmira, Colombia, were submitted by A. Villacorta, Cali, Colombia, on December 3, 1971. Diagnosis: Mycosis caused by Metarrhizium anisopliae.

\section{Spodoptera littoralis (Boisduval)}

2940 - An agar slant containing a fungus isolated from this noctuid was received from M. M. Altahtawy, Alexandria, Egypt, on February 12, 1970. Diagnosis: The fungus was identified as Spicaria rileyi. (Fig. 24,) 


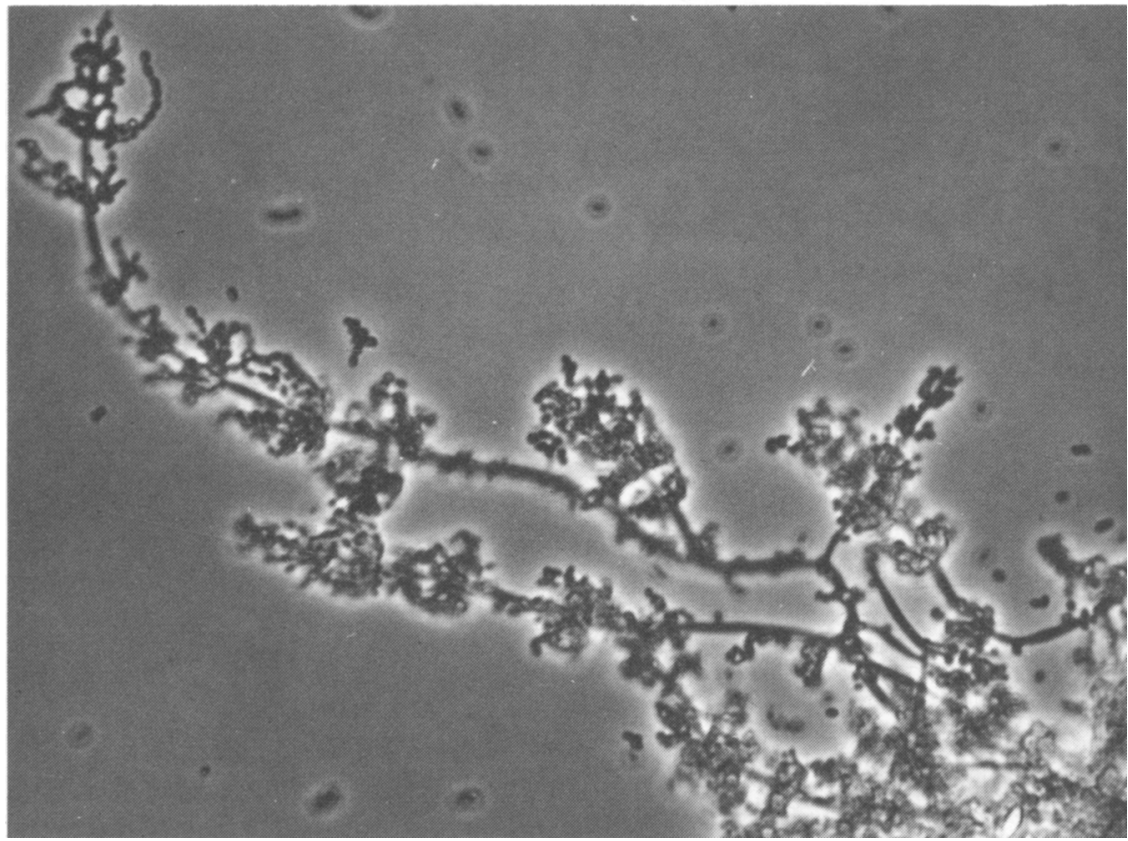

Fig. 24. Acc. No. 2940: Phase contrast micrograph of sporulating Spicaria rileyi isolated from Spodoptera littoralis. Magnification $=300 \mathrm{X}$.

2949-One larva was received from M. M. Altahtawy, Alexandria, Egypt, on April 23, 1970. The larva was covered with a thick mycelial mat producing light green spores. Diagnosis: Mycosis caused by Spicaria rileyi.

3026-A number of laboratoryreared larvae were submitted by M. M. Altahtawy, Alexandria, Egypt, on November 19, 1971. Diagnosis: Virosis caused by a nuclear polyhedrosis virus.

Spodoptera mauritia ssp. acconyctoides

2786-Three dead larvae were submitted on November 6, 1967 by G. L. H. Rothschild, Kuching, Sarawak, East Malaysia. Diagnosis: Mycosis caused by Spicaria rileyi.

\section{Tiracola plagiata (Walker)}

1839-These specimens were submitted by J. J. H. Szent-Ivany, Port Moresby, Territory of Papua and New Guinea, on February 8, 1962. Diagnosis: Non-microbial.
1844-Additional specimens were submitted by A. Catley, Port Moresby, Territory of Papua and New Guinea, on February 23, 1962. Diagnosis: Mycosis caused by Spicaria rileyi.

1845-_Some specimens were also submitted by A. Catley, Port Moresby, Territory of Papua and New Guinea, on February 25, 1962. Diagnosis: No definite pathogen found. Flavobacterium rhenanum was isolated.

1846-These specimens were submitted by F. C. Henderson, Port Moresby, Territory of Papua and New Guinea, on March 7, 1962. Diagnosis: Mycosis caused by Spicaria rileyi.

\section{Trichoplusia ni (Hübner)}

1963-Several specimens were submitted by J. R. Walker, Baton Rouge, Louisiana, on October 8, 1962. Diagnosis: Mycoses caused by Spicaria rileyi and Entomophthora megasperma.

2221-These specimens were sub- 
mitted by A. Sequeira D., Managua, Nicaragua, on January 17, 1964. Diagnosis: Mycosis caused by Spicaria rileyi.

2314-Several specimens received on September 10, 1964, from R. C. Hunter, Palmira, Valle, Colombia, were covered with white mycelium and conidia. The larval bodies were mummified and completely colonized by the fungus. Diagnosis: Mycosis caused by Beauvaria bassiana.

Trichoplusia ni (Hübner) or Pseudoplusia includens (Walker)

2320-Three field-collected looper larvae were submitted by $\mathrm{K}$. Elsey, Raleigh, North Carolina, on September 18,1964 . The specimens were covered with mycelium and light green spores. Diagnosis: Mycosis caused by Spicaria rileyi.

\section{Trichoplusia ni (Hübner)}

2658-Several larvae on artificial diets were submitted by C. Reiner, Berkeley, California, on July 27, 1966. The containers were contaminated with a variety of fungi. Identifications: Aspergillus niger, $A$. ochraceous, $A$. flavus, and a Penicillium sp.

2852_On August 2, 1968, several laboratory-reared larvae were received from J. J. Ellington, Las Cruces, New Mexico. Diagnosis: Virosis caused by a nuclear polyhedrosis virus.

2981-Five larvae of the cabbage looper were submitted by G. Leon Quant, Leon, Nicaragua, on February 3,1971 . The larvae had been collected in the field and reared in the laboratory. Each was infected with a different fungus. Diagnoses: Mycoses caused by Aspergillus flavus, Spicaria rileyi, Entomophthora sp., a Penicillium sp. (tested against Galleria melonella larvae for pathogenecity with positive results), and one unidentified fungus (deterioration precluded identification in this case).
2994-Four larvae of the cabbage looper were submitted by L. Falcon; from Managua, Nicaragua, on May 27, 1971. Diagnosis: Mycosis caused by Spicaria rileyi.

3035-Many dead larvae of the cabbage looper from soybeans in Cauca Valley, Colombia, were submitted by A. Villacorta, Cali, Colombia, on December 3, 1971. Diagnosis: Mycosis caused by Spicaria rileyi.

\section{Unidentified}

1841-These specimens were submittcd by F. McAlister, Clemson College, South Carolina, on February 13, 1962. Diagnosis: Mycosis caused by Spicaria rileyi.

1866-These specimens were collected during an epizootic above Placerville, California, and submitted by C. Gonzales, Berkeley, California, on April 19, 1962. Diagnosis: Virosis caused by a nuclear polyhedrosis virus.

Unidentified (subterranean caterpillar)

1949-These specimens were submitted by G. A. H. Helson, Wellington, New Zealand, on September 18, 1962. Diagnosis: Mycosis caused by Entomophthora sp.

1959-Additional specimens were submitted by G. A. H. Helson, Wellington, New Zealand, on October 1, 1962. Diagnosis: Mycoses caused by Entomophthora sp. (near aphidis) and Cordyceps sp.

1982-These specimens were also submitted by G. A. H. Helson on November 21, 1962. Diagnosis: Insect parasitism.

2628-Several larvae were received from C. S. Koehler, Berkeley, California, on June 1, 1966. Diagnosis: Infection by Streptococcus sp. as a potential pathogen or secondary invader. Primary etiology undetermined, but evidently non-microbial. 
2978-Four larvae were received on December 28, 1970, from S. B. Mathad, Dharwar, Mysore State, India. The specimens were collected from Sorgum vulgare. Diagnosis: Virosis caused by a nuclear polyhedrosis virus.

\section{Notodontidae}

Schizura concinna (J. E. Smith)

2686-Several larvae were received from J. E. Dibble, Berkeley, California, on September 20, 1966. Diagnosis: Non-microbial.

\section{Nyctemeridae}

Tyria jacobaea (L.)

2831-Many pupae of the cinnabar moth were submitted by $R$. Hawkes, Albany, California, on May 22, 1968. The pupae were collected in fields of tansy ragwort at Fort Bragg, California. Diagnosis: Microsporidiosis caused by a Nosema sp.

2842_Additional specimens (eggs and larvae), collected near Todd Point, Fort Bragg, California were submitted by R. Hawkes on June 24, 1968. Diagnosis: Microsporidiosis caused by a Nosema sp.

2845-Another collection of larvae from Senecio jacobaea (tansy ragwort) near Todd Point, Fort Bragg, California, was submitted by R. Hawkes on July 1, 1968. Diagnosis: Microsporidiosis caused by a Nosema sp.

2956-_Several larvae were submitted on July 17, 1970, by R. Hawkes, Albany, California. These larvae were reared in the laboratory from eggs collected near Fort Bragg, California. Unusually high mortality was expericnced in the laboratory cultures. Diagnosis: Microsporidiosis caused by $\mathrm{No}$ sema sp.

\section{Nymphalidae}

Caligo sp., probably eurilochus

2638-One larva was received from

F. Lara E., Limon, Costa Rica, on
June 21, 1966. Diagnosis: Bacteriosis: Streptococcus faecalis.

\section{Limenitis archippus (Cramer)}

2989-Two deteriorated larvae of the viceroy butterfly were received on May 31, 1971, from Jo Brewer, Auburndale, Massachusetts. The larvae had been collected in Maine for rearing. Diagnosis: Bacterioses caused by Bacillus cereus and B. cereus var. $m y$ coides.

\section{Nymphalis antiopa (L.)}

2299-Several larvae were received from J. D. Stein, Fargo, North Dakota, on July 10, 1964. Diagnosis: Nonmicrobial-possible parasitism with secondary mycosis by Fusidium sp. and bacteriosis by Aerobacter sp. (probably $A$. liquefacens).

\section{Nymphalis californica (Boisduval)}

2990-One dead larva of the California tortoise shell butterfly was submitted by W. E. Ferguson, San Jose, California, on May 19, 1971. The specimen was dark brown and soft, but intact. Diagnosis: Bacteriosis caused by Bacillus megaterium.

\section{Speyeria nokomis (Edwards)}

2930_On December 18, 1969, a few larvae of the nokomis fritillary collected from Viola priceana and $V$. papilionacea were submitted by S. O. Mattoon, Chico, California. The specimens had been collected in Ruby Valley, Elko County, Nevada, and reared in Chico. Diagnosis: Non-microbial.

\section{Vanessa cardui (L.)}

2937-On February 12, 1970, C. White, Shafter, California, submitted four laboratory-reared larvae of the painted lady butterfly. Diagnosis: Nonmicrobial.

\section{Vanessa virginensis (Drury) 2961-J. Brewer, Auburndale, Mas-}


sachusetts, submitted a specimen of the painted beauty butterfly on August 31, 1970. The specimen was collected from Gnaphalium sp. in Georgetown, Massachusetts, and was the only dead larva found in a high population of the insects. Diagnosis: Non-microbial.

\section{Olethreutidae}

Carpocapsa pomonella (L.)

1960 - Several laboratory-reared specimens of codling moth larvae were submitted by S. C. Hoyt, Wenatchee, Washington, on October 2, 1962. Diagnosis: Bacterioses caused by Bacillus megaterium and Aerobacter cloacae.

2070-These specimens were submitted by L. Falcon, Berkeley, California, on June 13, 1963. Diagnosis: Undetermined, but probably nonmicrobial.

2075-One larva was submitted by L. Falcon, Berkeley, California, on June 20, 1963. The area of collection had been treated with Thuricide 90T on May 17, 1963. Diagnosis: Bacteriosis caused by Bacillus thuringiensis.

2080-These specimens were collected in Linden, California, and submitted by L. Falcon, Berkeley, California, on July 5,1963 . They came from an area that had been treated with Thuricide 90T. Diagnosis: Bacteriosis caused by Bacillus thuringiensis.

2163-One additional specimen collected in Linden, California, was submitted by L. Falcon on September 11, 1963. The area had been treated with Bacillus thuringiensis. Diagnosis: Bacteriosis caused by Bacillus thuringiensis.

2166-These specimens were submitted by L. E. Caltagirone, Albany, California, on September 17, 1963. They had been collected in Chihuahua, Mexico. Diagnosis: Nematode infection: Neoaplectana sp. (probably another strain of $N$. carpocapsae)
2180 - A dead larva was submitted by L. A. Falcon, Berkeley, California, on October 7,1963 . It had also been collected from Linden, California. Diagnosis: Probably non-microbial. The bacteria found were saprophytic.

2186-Four larvae collected near Valle de Allende, Chihuahua, Mexico, were submitted by L. Caltagirone, Berkeley, California, on October 17, 1963. Diagnosis: Some suffered a mycosis caused by Beauveria bassiana; others were infected with a granulosis virus. The latter is a new record for this host.

2459-These laboratory-reared specimens were submitted by W. Kane, Berkeley, California, on August 19, 1965. Diagnosis: Non-microbial.

2501-Additional laboratory reared specimens were submitted by W. Kane, Berkeley, California, on October 25, 1965. Diagnosis: Non-microbial.

2555-S€veral more specimens were submitted by W. Kane. These were collected in Placerville, California, on October 26, 1965. Diagnosis: Mycosis: Beauveria bassiana.

2714 - Several larvae were submitted by R. D. McMullen, Summerland, British Columbia, on December 20, 1966. Diagnosis: Virosis: granulosis virus.

2807-Several dead larvae were submitted on January 23, 1968 by J. A. Quist, Grand Junction, Colorado. Diagnosis: Virosis caused by a granulosis virus.

2939_On March 4, 1970, several larvae of the codling moth from mass rearings on an artificial diet were submitted by M. D. Proverbs, British Columbia, Canada. Diagnosis: Virosis caused by a granulosis virus.

3038-Larvae and pupae were submitted by J. M. Jordon, Farmington, New Mexico. The specimens had died while overwintering in corrugated cardboard bands. Diagnosis: Mycosis caused by Beauveria bassiana. 
1849-These specimens were submitted by M. A. Ghani, Rawalpindi, Pakistan, on March 12, 1962. Diagnosis: Possible mycosis caused by Fusarium sp.

\section{Grapholitha molesta (Busk)}

2278-Several larvae originally from Japan were submitted by. G. Finney, Berkeley, California, on June 12 and July 3, 1964. Diagnosis: Probably nonmicrobial, but if the primary etiology was microbial it has been obscured by the action of secondary saprophytic bacteria.

2803-On February 2, 1968, R. Laing, Berkeley, California, submitted three laboratory-reared larvae of the oriental fruit moth. Diagnosis: Nonmicrobial.

\section{Rhyacionia bouliana (Schiff.)}

2824-Several dry laboratory-reared larvae and pupae of the European pine shoot moth were submitted by S. S. Chawla, Pullman, Washington, on May 8, 1968. Diagnosis: Bacteriosis caused by Bacillus cereus. This bacterium was isolated in pure culture from externally sterilized specimens, indicating contamination of the rearing medium and possible stress conditions.

2849_Pupae were again submitted by S. S. Chawla on July 17, 1968. Diagnosis: Non-microbial.

2889--Additional larvae, pupae, and adults were submitted by S. S. Chawla on March 31, 1969. Diagnosis: Bacterioses caused by the potential pathogens Bacillus cereus and Streptococcus faecalis.

\section{Rhyacionia pasadenana (Kearfott)}

1973-Several specimens were submitted by C. S. Koehler, Berkeley, California, on October 16, 1962. Diagnosis: Non-microbial.

2166A-Additional specimens were submitted by C. S. Koehler, Berkeley,
California, on September 19, 1963. Diagnosis: Non-microbial.

\section{Rhyacionia zozana (Kearfott)}

2231-Some specimens from Placerville, California, were submitted by R. E. Stevens, Berkeley, California, on February 14, 1964. Diagnosis: Mycosis caused by Beauveria bassiana. New record.

2244-Several more specimens were collected 15 miles north of Groveland, Tuolome County, California, by R. E. Stevens on March 26, 1964. Diagnosis: Non-microbial.

2253-Additional specimens were collected in Placerville, California, by R. E. Stevens on April 17, 1964. Diagnosis: Parasitism by an Ichneumonid parasite.

\section{Papilionidae}

Papilio ajax (L.)

2737-One pupae was submitted by J. Brewer, Auburndale, Massachusetts, on April 11, 1967. Diagnosis: Nonmicrobial.

\section{Papilio polyxenes asterius Stoll}

2850_On July 24, 1968, one larva of the black swallowtail butterfly from parsley was received from J. Brewer, Auburndale, Massachusetts. Diagnosis: Non-microbial.

2960-One larva was submitted on August 16, 1970 by J. Brewer, Auburndale, Massachusetts. The specimen was collected from parsley in Newton, Massachusetts, where there was evidently an epizootic of the disease. Dignosis: Microsporidiosis caused by a Nosema sp.

\section{Papilio sp.}

3018-One dead larva was submitted by M. Thomas, Berkeley, California, on September 28, 1971. Diagnosis: Nonmicrobial. 


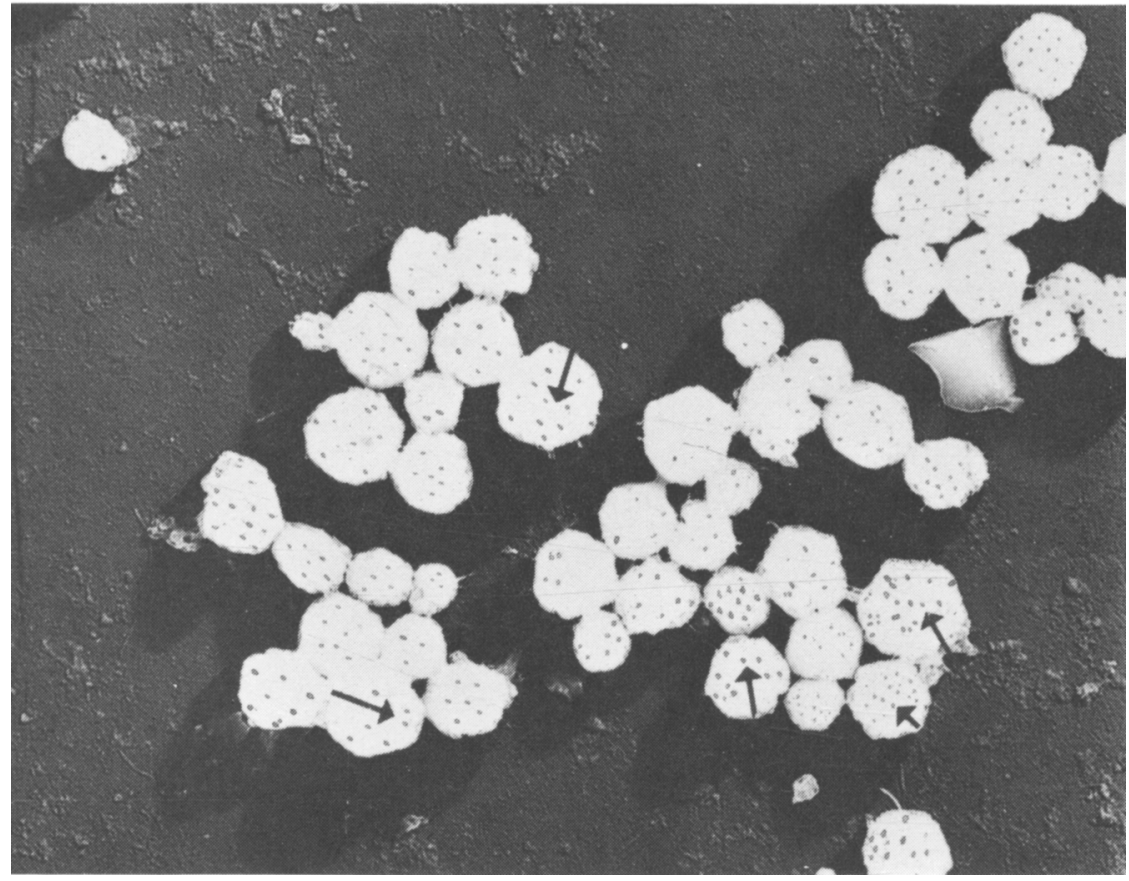

Fig. 25. Acc. No. 2023 : Electron micrograph of polyhedra of a cytoplasmic polyhedrosis virus from Cactoblastis cactorum. The pits containing the spherical virus particles can be seen in some of the polyhedra (arrows). Magnification $=14,500 \mathrm{X}$.

\section{Phycitidae}

\section{Anagasta kuhniella (Zeller)}

2024-Bacterial cultures were isolated and submitted by P. A. van der Laan, Amsterdam, Holland, on March 11, 1963. Identification: Bacillus thuringiensis.

2577-Several specimens were submitted by C. Reiner, Albany, California, on March 10, 1966. Diagnosis: Bacillus thuringiensis var. thuringiensis.

3023-Several larvae were submitted by G. King, Albany, California, on October 10, 1971. Diagnosis: Non-microbial.

\section{Cactoblastis cactorum (Berg)}

2023-Eight laboratory-reared larvae were submitted by M. Tamashiro, Honolulu, Hawaii, on March 6, 1963. Diagnosis: Virosis caused by a cyto- plasmic polyhedrosis virus. New record. (Fig. 25.)

\section{Ephestia figulilella Gregson}

1889 - A culture of raisin moths was received from G. H. Spitler, Fresno, California, on June 12, 1962. A disease was causing an unusual amount of mortality in their laboratory colonies. Diagnosis: Bacteriosis caused by Bacillus thuringiensis var. thuringiensis. New record.

\section{Paramyelois transitella (Walker)}

1891-On June 15, 1962, a culture of the navel orangeworm was submitted by M. Husseiny, Berkeley, California. Many of the larvae in the culture were dying and turning black. Diagnosis: Bacteriosis caused by Bacillus entomocidus (B. thuringiensis var. entomoci$d u s)$. New host record.

1974-These laboratory-reared spec- 
imens were submitted by D. Price, Davis, California, on October 23, 1962. Diagnosis: Microsporidiosis caused by Nosema sp. possibly destructor. New record.

1986-These specimens were submitted by L. A. Falcon, Berkeley, California, on December 7, 1962. Diagnosis: Non-microbial.

1996-A sample of larvae was submitted by J. A. Goodwin, Berkeley, California, on December 19, 1962. These larvae were from a laboratory culture of about 400 infected navel orangeworms. Diagnosis: Bacteriosis caused by Bacillus thuringiensis var. entomocidus.

2005A-Several specimens were submitted by L. A. Falcon, Berkeley, California, on January 23, 1963. Diagnosis: Probably non-microbial.

2012-These laboratory-reared specimens were submitted by G. Finney, Albany, California, on February 12, 1963. Diagnosis: Bacteriosis caused by Bacillus entomocidus.

2083-These specimens were collected in Linden, California and submitted by L. A. Falcon, Berkeley, California, on July 11, 1963. Diagnosis: No pathogens found.

2162-Additional specimens collected in Linden, California, were submitted by L. A. Falcon on September 11, 1963. Diagnosis: Non-microbial.

2181-One larva was submitted by L. A. Falcon on October 7, 1963. Diagnosis: Probably non-microbial.

2213-Several specimens were submitted by F. M. Summers, Davis, California, on December 17, 1963. Diagnosis: No pathogens found.

2345 - Two dead larvae were submitted on December 18, 1964, by I. H. Collins, Davis, California. The insects were reared in the laboratory on an artificial diet. Diagnosis: Bacteriosis caused by Bacillus thuringiensis var. thuringiensis.
2346-Several laboratory-reared larvae were submitted by $D$. Brinkman, Berkeley, California, on January 7, 1965. Diagnosis: Bacteriosis: Bacillus cereus var. mycoides and a schizogregarine protozoan infection, possible Mattesia $\mathrm{sp}$.

2350-These larvae were submitted by K. Shey, Berkeley, California, on January 18, 1965. The primary cause of death was probably non-microbial, but it had been obscured by a secondary bacteriosis. Diagnosis: Bacterioses: Pseudomonas aeruginosa, Streptococcus faecalis, and Alcaligenes faecalis.

2476-Laboratory-reared specimens were submitted by U. Gogia, Berkeley, California, on September 20, 1965. Diagnosis: Bacteriosis caused by Bacillus thuringiensis var. thuringiensis.

2585-Ten dead larvae were submitted by L. Caltagirone, Berkeley, California, on March 23, 1966. Diagnosis: Bacteriosis caused by Bacillus thuringiensis var. thuringiensis.

2586-Several dead specimens were submitted by L. Caltagirone on March 30, 1966. Diagnosis: Bacillus thuringiensis var. thuringiensis. Parasitized specimens were also infected.

2605-These laboratory-reared specimens were submitted by D. Brinkman, Berkeley, California, on April 28, 1966. Diagnosis: Protozoan infection caused by the schizogregarine, Mattesia dispora.

2715-Several specimens were received from L. A. Falcon, Berkeley, California, on January 3, 1967. Diagnosis: Bacteriosis caused by Bacillus thuringiensis var. thuringiensis.

2777-Several dead laboratoryreared larvae were submitted by $D$. Brinkman, Albany, California, on October 4, 1967. Diagnosis: Bacteriosis caused by Bacillus thuringiensis var. thuringiensis.

2892-Several laboratory-reared larvae were submitted by $D$. Brinkman, 


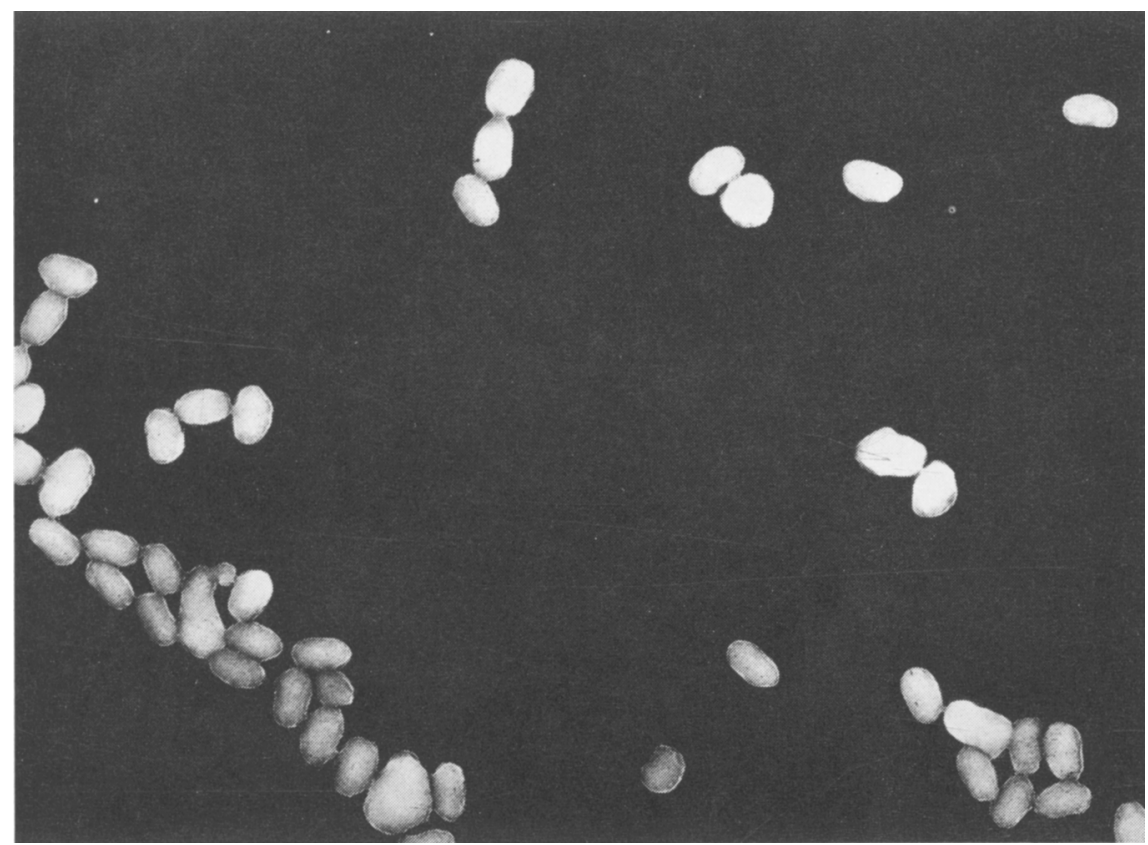

Fig. 26. Acc. No. 2316: Electron micrograph of granulosis virus capsules from Plodia interpunctella. Magnification $=16,200 \mathrm{X}$.

on April 1, 1969. Diagnosis: Protozoan infection caused by the schizogregarine, Mattesia dispora.

3019-Several dead larvae were submitted by D. Pinnock, Berkeley, California, on October 4, 1971. The specimens had been collected from almonds in La Grand and Arbuckle, California. Diagnosis: Non-microbial.

\section{Plodia interpunctella (Hübner)}

2309 - Several laboratory-reared specimens were submitted by G. $H$. Spitler, Fresno, California, on August 27, 1964. Diagnosis: Bacteriosis caused by Bacillus thuringiensis and a protozoan infection caused by Mattesia dispora.

2316-Several dead laboratoryreared larvae were submitted on September 10, 1964, by W. E. Burkholder, Fresno, California. Diagnosis: Virosis caused by a granulosis virus. New record. (Fig. 26.)

2883-On February 4, 1969, J. A.
Reinert, Clemson, South Carolina, submitted several laboratory-reared larvae. Diagnosis: Virosis caused by a granulosis virus.

\section{Vogtia sp.}

2871-On December 2, 1968, one larva, one pupa, and one adult from a laboratory culture were submitted by D. Maddox, Albany, California. Diagnosis: Non-microbial.

\section{Pieridae \\ Colias lesbia Fab.}

2565-Thirteen dead specimens collected between Lujan and Pergamino Buenos Aires, Argentina, on January 24, 1966, were submitted by I. S. de Crouzel on February 2, 1966. Diagnosis: Microsporidiosis caused by a $\mathrm{No}$ sema near polyvora and polyhedrosis caused by a nuclear polyhedrosis virus.

2740-Several more larvae were submitted by I. S. de Crouzel, Buenos Aires, Argentina, on April 19, 1967. 


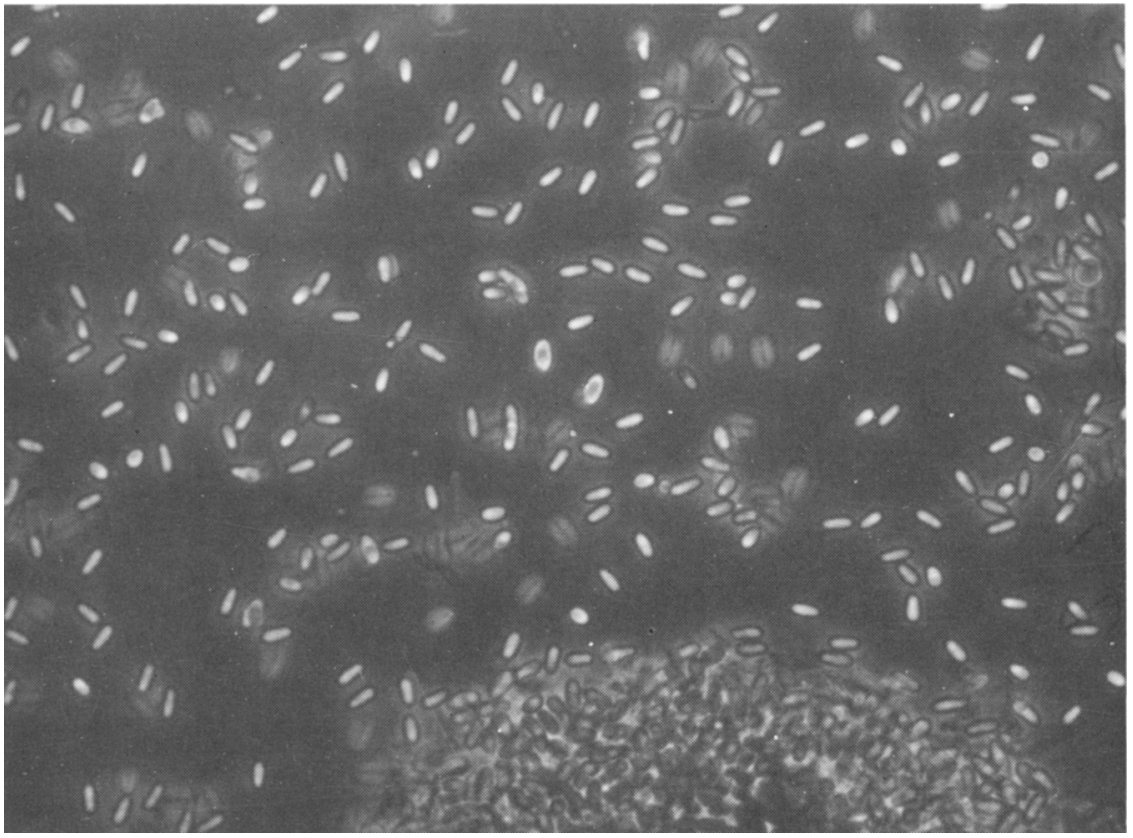

Fig. 27. Acc. No. 2755: Phase contrast of Nosema coliadis spores from Colias lesbia. Magnification $=800 \mathrm{X}$.

Diagnosis: Microsporidiosis caused by Nosema coliadis.

2750-Four additional larvae were submitted by I. S. de Crouzel, Buenos Aires, Argentina, on May 4, 1967. Diagnosis: Microsporidiosis caused by Nosema coliadis.

2755-Several more specimens were submitted by I. S. de Crouzel on June 7, 1967. Diagnosis: Microsporidiosis caused by Nosema coliadis. (Fig. 27.)

2758-Several more specimens were submitted by I. S. de Crouzel on July 7, 1967. Diagnosis: Microsporidiosis caused by Nosema coliadis.

\section{Delias belladonna Fab.}

2818-Some pupae and larvae collected from sandal wood trees in Dehra Dun, India, were submitted by P. N. Chatterjee on April 3, 1968. Diagnosis: No primary microbial pathogens were found, but microbial analysis revealed the potential pathogen, Streptococcus faecalis, as well as a secondary invader, Aerobacter sp.

\section{Pieris brassicae (L.)}

2480 - These specimens were submitted by R. A. Malik, Kashmir, India, on September 27, 1965. Diagnosis: Bacteriosis caused by Streptococcus faecalis.

2935-Larvae, pre-pupae, and pupae were received from $\mathrm{O}$. S. Bindra, Ludhiana, India, on February 10, 1970. Diagnosis: Bacteriosis caused by the potential pathogen, Streptococcus faecalis.

\section{Pieris rapae (L.)}

2884 Two larvae of the alfalfa butterfly were submitted by J. M. Kolyer, Convent, New Jersey, on March 10, 1969. These larvae were from a laboratory culture which suffered extensive mortality. Diagnosis: Bacteriosis caused by Serratia marcescens. 


\section{Plutellidae}

Abebaea subsylvella (Walsingham)

2248_-These specimens were submitted by W. Brooks, Berkeley, California, on April 7, 1964. The area had been sprayed with Malathion. Diagnosis: Toxicosis caused by Malathion.

\section{Psychidae}

Metisa plana (Wlk.)

2262--Several dead larvae were submitted by B. J. Wood, Johore, Malaya, on May 13, 1964. Diagnosis: Bacteriosis caused by Bacillus cereus.

\section{Oiketycus kirbyi (Guilding)}

2371-Larvae of the banana bagworm were collected in La Estrella Valley by F. Lara E., Limon, Costa Rica, on March 24, 1965. Two of the specimens were collected before the area was treated with Bacillus thuringiensis and the rest were collected afterwards. Diagnosis: Bacterioses caused by Bacillus cereus and Streptococcus sp. in the untreated specimens. Bacteriosis caused by Bacillus thuringiensis in the treated specimens.

2379-Additional specimens were collected in La Estrella Valley, Costa Rica, on March 17, 1965, by F. Lara E. Diagnosis: Bacteriosis caused by Bacillus thuringiensis.

2389-These specimens from the Plantacion "San Jose," Buenos Aires, Argentina, were submitted by $\mathrm{H}$. Stjernfalk on March 25, 1965. The area had been sprayed with Thuricide 90 . Diagnosis: Bacteriosis caused by Bacillus thuringiensis.

2422-These specimens were submitted by C. S. Stephens, Changuinola, Panama, on June 22, 1965. Diagnosis: Mycoses caused by Beauveria bassiana and Aspergillus flavus.

2567-One dead pupa was submitted by C. S. Stephens, Changuinola, Panama, on February 14, 1965. Diagnosis: Non-microbial.
2745-Two larvae were submitted by C. S. Stephens on April 20, 1967. Diagnosis: Virosis caused by a cytoplasmic polyhedrosis virus.

\section{Oiketicus pratensis Berg}

2763--Several larvae were submitted by I. S. de Crouzel, Buenos Aires, Argentina, on July 7, 1967. Diagnosis: Bacteriosis caused by a Bacillus sp.

\section{Oiketicus sp.}

2810_On February 26, 1968, four larvae of the banana bagworm were submitted by I. S. de Crouzel, Castelar, Argentina. Diagnosis: Bacteriosis caused by a Bacillus sp., with a secondary infection by a Streptococcus sp.

\section{Pyralidae}

Acrobasis tricolorella (Grote)

2749-Several larvae were submitted by P. E. Boldt, East Lansing, Michigan, on May 5, 1967. Diagnosis: Non-microbial. Fungi present were a Penicillium sp., Alternaria sp., and a Hormodendrum sp. There was evidence of parasitism in a few specimens.

\section{Chilotraea infuscatella (Snellen)}

1942-A bacterial and fungal culture isolated from this pyralid were received from Wen-Yi-Cheng, Tainan, Taiwan, on September 12, 1962. Diagnosis: Bacteriosis caused by Serratia marcescens and mycosis caused by Metarrhizium anisopliae.

\section{Desmia funeralis (Hübner)}

2891-Several laboratory-reared larvae of the grape leaf roller were submitted by D. Brinkman, Berkeley, California, on April 1, 1969. Diagnosis: Protozoan infection caused by the schizogregarine, Mattesia dispora.

\section{Herculia nigrivitta (Walker)}

1944_Five cultures isolated from this insect were submitted by Feng 
Ying Cheng, Tainan, Taiwan, on September 12, 1962. Diagnosis: Metarrhizium anisopliae, Serratia marcescens, Pseudomonas aeruginosa and an unidentified yeast.

\section{Mineola scitulella (Hulst.)}

2681-Two specimens were received from P. Boldt, East Lansing, Michigan, on September 8, 1966. Diagnosis: Nonmicrobial but both were covered with saprophytic Dematiaceae.

\section{Nacoleia diemenalis (Guen)}

2402-These larvae were submitted by S. Rao, Selagor, Malaya, on May 10, 1965. Diagnosis: Virosis caused by granulosis virus. New record.

\section{Nacoleia octasema (Meyrick)}

2049 - Several laboratory-reared specimens were submitted by $R$. W. Paine, Honiara, British Solomon Islands, on May 2, 1963. Diagnosis: Probably non-microbial.

\section{Sceliodes laisalis}

2325 - A single larva was submitted by T. V. Venketraman, Khartoum, Republic of the Sudan, on October 5, 1964. Diagnosis: Non-microbial.

\section{Tryporyza incertulus (Walker)}

2970 - Three dead specimens of the rice stem borer collected in the Swat Valley and Kala Shah Kaku, Pakistan, were submitted by C. S. Koehler, Berkeley, California. Diagnosis: $\mathrm{My}$ cosis caused by a Fusarium sp.

\section{Tryporyza innotata (Walker)}

2732-Two specimens of the white rice borer larvae were received from C. S. Li, Darwin, Australia, on March 3, 1967. Diagnosis: No primary pathogens present. Cause of death was probably from a Lindane treatment.

\section{Pyraustidae}

\section{Ostrinia kasmirika}

2979-Six laboratory-reared specimens were submitted by A. S. Atwal, Ludhiana, India. Diagnosis: Bacteriosis caused by Serratia marcescens.

\section{Ostrinia nubialis (Hübner)}

1883-These specimens were submitted by R. D. Frye, Fargo, North Dakota, on June 1, 1962. Diagnosis: Microsporidiosis caused by Glugea $p y$ raustae.

\section{Saturniidae}

Antheraea assamensis (Helfer)

2969-Several dried pupae of the Muga silkworm were received on October 20, 1970, from J. N. Talukdar, Assam, India. The specimens were collected from Som plant (Machilus oderatissima) and Soalu plant (Tetranthera monopetala) near Assam, where about 20 per cent of the insect population was infected. Diagnosis: Microsporidiosis caused by a Nosema sp.

\section{Antheraea eucalypti Scott}

1907-One specimen was submitted by G. A. H. Helson, Wellington, New Zealand, on July 24, 1962. Diagnosis: Mycosis caused by Beauveria bassiana.

Antheraea morosa Butler $x$ A. pernyi (Guérin-Meneville)

2806-Two larvae of the above genetic cross were submitted by $\mathrm{H}$. Flaschka, Atlanta, Georgia, on February 13, 1968. Diagnosis: Non-microbial.

\section{Antheraea mylitta Drury}

2881--An aqueous suspension of decomposed tissues from this tassor silkworm was submitted by M. S. Jolly, Ranchi, India, on February 25, 1969. Diagnosis: Microsporidiosis, caused by Nosema bombycis.

3022-Several larvae collected from 
Terminalis tomentosa, were submitted by S. B. Mathad, Mysore, India. Diagnosis: Virosis caused by a nuclear polyhedrosis virus.

\section{Antheraea polyphemus (Cramer)}

1953-These laboratory reared specimens were submitted by R. D. Frye, Fargo, North Dakota, on September 25, 1962. Diagnosis: Undetermined. Possible bacteriosis caused by Flavobacterium sp.

\section{Attacus ricini Pagenstecher}

2566-Cultures were submitted by M. M. Altahtawy, Alexandria, U.A.R., on January 9, 1966. Diagnosis: Mycosis caused by Aspergillus flavus. Also present were Aspergillus ochraceus and Aspergillus niger.

2624-A fungal culture and vial of fungal spores were received from ElSayed Megahed, Alexandria, U.A.R., on May 13, 1966. Diagnosis: Aspergillus lutescens. Tests against wax moth larvae were negative, however.

2625-Another fungal culture and vial of spores were received from $M$. Altahtawy on May 23, 1966. Diagnosis: Vial 1 (Agar Slant)_Scopulariopsis sp. Vial 2a-Aspergillus flavus-oryzae group which seems to be intermediate between $A$. flavus Link and $A$. effusus. $2 \mathrm{~b}-A$. Alavus-oryzae group, closer to A. parasiticus than to any of the others. Tests against Galleria larvae gave negative results.

\section{Automeris sp.}

1962 - Several laboratory-reared specimens were submitted by B. O. C. Gardiner, Cambridge, England, on October 4, 1962. Diagnosis: Mycosis caused by Beauveria bassiana.

\section{Coloradia pandora (Blake)}

2354 - G. Downing, Berkeley, California, submitted several specimens collected near Chemult, Oregon, on
February 9, 1965. Original specimens indicated a granulosis virus, but infectivity tests failed to confirm these results. Diagnosis: Inconclusive.

\section{Dirphia amphimome (Fabricus)}

2212-Several specimens collected in southern Chile were submitted by G. Olalquiaga, Santiago, Chile, on December 16, 1963. Diagnosis: Mycosis caused by Entomophthora grylli. New record.

\section{Hemileuca maia (Drury)}

1988-These specimens were submitted by A. D. Oliver, Baton Rouge, Louisiana, on December 10, 1962. Diagnosis: Bacteriosis caused by Bacillus cereus.

2046-Laboratory-reared specimens were again submitted by A. D. Oliver on April 27, 1963. Diagnosis: General bacterioses involving a mixture of coliform bacteria.

\section{Hemileuca oliviae Cockerell}

2957-Seven diseased larvae of the range caterpillar were received on July 27, 1970 from G. L. Nielsen, Las Cruces, New Mexico. The larvae were collected from snake weed and various grasses. Diagnosis: Bacteriosis caused by Bacillus cereus.

3009-Larvae collected from Bermuda grass at Las Cruces, New Mexico, were submitted by J. G. Watts on August 30, 1971. Diagnosis: Bacteriosis caused by Bacillus cereus and Streptococcus faecalis.

\section{Hyalophora cecropia (L.)}

1901 - Several laboratory-reared specimens of the cecropia moth were submitted by R. D. Frye, Fargo, North Dakota, on July 20, 1962. Diagnosis: Non-microbial.

2096-Laboratory-reared larvae were submitted by Warren R. Cothran, Ithaca, New York, on August 7, 1963. Diagnosis: Mycosis caused by Aspergillus flavus. 


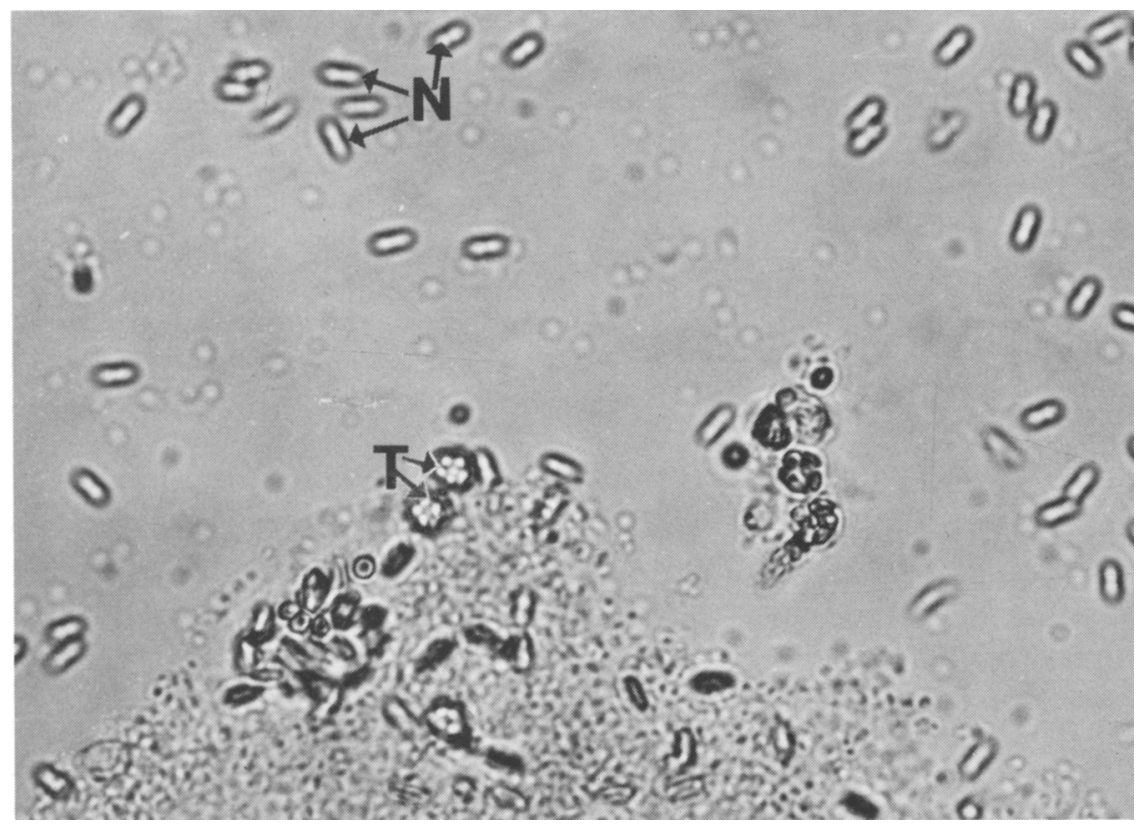

Fig. 28. Acc. No. 2772: Phase contrast of a double protozoan infection by a Nosema sp. and a Thelohania sp. in Hyalophora cecropia. Nosema spores (N); Thelohania pansporoblasts (T). Magnification $=800 \mathrm{X}$.

2421-These laboratory-reared specimens were submitted by W. R. Harvey, Amherst, Massachusetts, on June 13, 1965. Diagnosis: Bacterioses caused by Bacillus cereus and Pseudomonas aeruginosa.

2596-Eggs were submitted by J. A. Haskell, Amherst, Massachusetts, on April 8, 1966. A thorough examination of triturated smears under phase and in Giemsa-stained preparations showed no apparent microbial etiology. Cultures set under both aerobic and anaerobic conditions from externally sterilized and triturated eggs gave negative results. Diagnosis: Non-microbial.

2772-Several dead larvae collected from apple leaves were received from S. Samuelson, Pleasantville, Iowa, on September 7, 1967. Diagnosis: Microsporidioses caused by Nosema sp. (Dominant) and a Thelohania sp. (Fig. 28.)

2959-On August 17, 1970, six larvae were received from G. B. Black, Pleas- antville, Iowa. These larvae were from a laboratory culture which had suffered considerable mortality. Diagnosis: Microsporidiosis caused by a Nosema sp. 2998 - Two larvae were submitted by J. Lowry, Prairie Village, Kansas. Some of the specimens in his culture exhibited symptoms of diarrhea, but the two larvae submitted were evidently in good health, and examinations showed no pathogens. Diagnosis: Non-microbial.

2999-Three dead pupae and one larvae from the culture of G. B. Black, Pleasantville, Iowa, were submitted on July 12, 1971. Diagnosis: Non-microbial.

\section{Hyalophora euryalus (Bdv.)}

2426 - Several laboratory-reared specimens were submitted by W. M. Brooks, Berkeley, California, on June 29, 1965. Diagnosis: Bacteriosis caused by Bacillus cereus. 


\section{Hyalophora rubra (Behr.)}

2825-Two laboratory-reared larvae were submitted by G. H. Flaschka, Atlanta, Georgia, on May 8, 1968. Diagnosis: Non-microbial.

\section{Hylesia nigricans Berg}

1961-B. O. C. Gardiner, Cambridge, England, submitted 14 larvae from his laboratory culture which was experiencing from 60 per cent to 100 per cent mortality. Diagnosis: Virosis caused by a nuclear polyhedrosis virus. This apparently is a new host record. (Fig. 29.)

\section{Rothschildia orizaba (Westwood)}

2007-These specimens from Mexico City were submitted by W. Brooks, Berkeley, California, on February 4, 1963. Diagnosis: Probably non-microbial.

\section{Samia cynthia (Drury)}

2452-These specimens were submitted from a laboratory culture by $\mathrm{F}$. Maglio, Oakland, California, on $\mathrm{Au}-$ gust 6, 1965. Diagnosis: Non-microbial.

2803-A few detiorated larvae were received from S. Samuelson, Pleasantville, Iowa, on May 21, 1968. Diagnosis: Non-microbial. Microbial analysis produced only a few saprophytic coliform bacteria.

2835-Additional larvae from Ailanthis were submitted by S. Samuelson on May 31, 1968. Diagnosis: The specimens were infected by a Streptococcus sp. and other secondary coliform bacteria. Any possible primary microbial etiology had been obscured by the action of these secondary invaders.

\section{Sphingidae}

\section{Herse convolvuli (L.)}

2324-A single larva was submitted by T. V. Venketraman, Khartoum, Republic of the Sudan, on October 5, 1964. Diagnosis: Probable bacteriosis caused by Bacillus cereus.
Manduca sexta (Johann.)

2744-One larva was submitted by M. W. McFadden, Oxford, North Carolina, on April 25, 1967, requesting confirmation of possible cytoplasmic polyhedrosis. Diagnosis: A check for cytoplasmic polyhedrosis and other viruses was negative.

\section{Pachyspinx modesta (Harris)}

1954-Laboratory-reared specimens were submitted by R. D. Frye, Fargo, North Dakota, on September 25, 1962. Diagnosis: Undetermined. Flavobacterium sp. was present, but its relationship to the insect is unknown.

\section{Protoparce quinquemaculata (Haw.)}

3001-One dead larva from the controls of a laboratory experiment with Bacillus thuringiensis was submitted on July 27, 1971 by D. Pinnock, Berkeley, California. Diagnosis: Bacteriosis caused by Pseudomonas aeruginosa.

\section{Protoparce sexta (Johannson)}

1834 - These specimens were submitted by R. L. Rabb, Raleigh, North Carolina, on January 17, 1962. Diagnosis: Bacterioses caused by Clostridium saprogenes and Bacillus cereus.

2403-Several larvae collected in Florence, South Carolina, were submitted by S. D. Carlson, Blacksburg, Virginia, on May 12, 1965. Diagnosis: Probably non-microbial with Penicillium sp., Rhizopus sp., and Streptococcus $\mathrm{sp}$. as secondary invaders.

\section{Hornworm}

2414-Several dead larvae were submitted by S. D. Carlson, Blacksburg, Virginia, on June 7, 1965. The larvae were field-collected and then stored under refrigeration. Diagnosis: Bacteriosis caused by Bacillus cereus, Streptococcus faecalis, and a Proteus sp.

2545-One dead, deteriorated spec- 

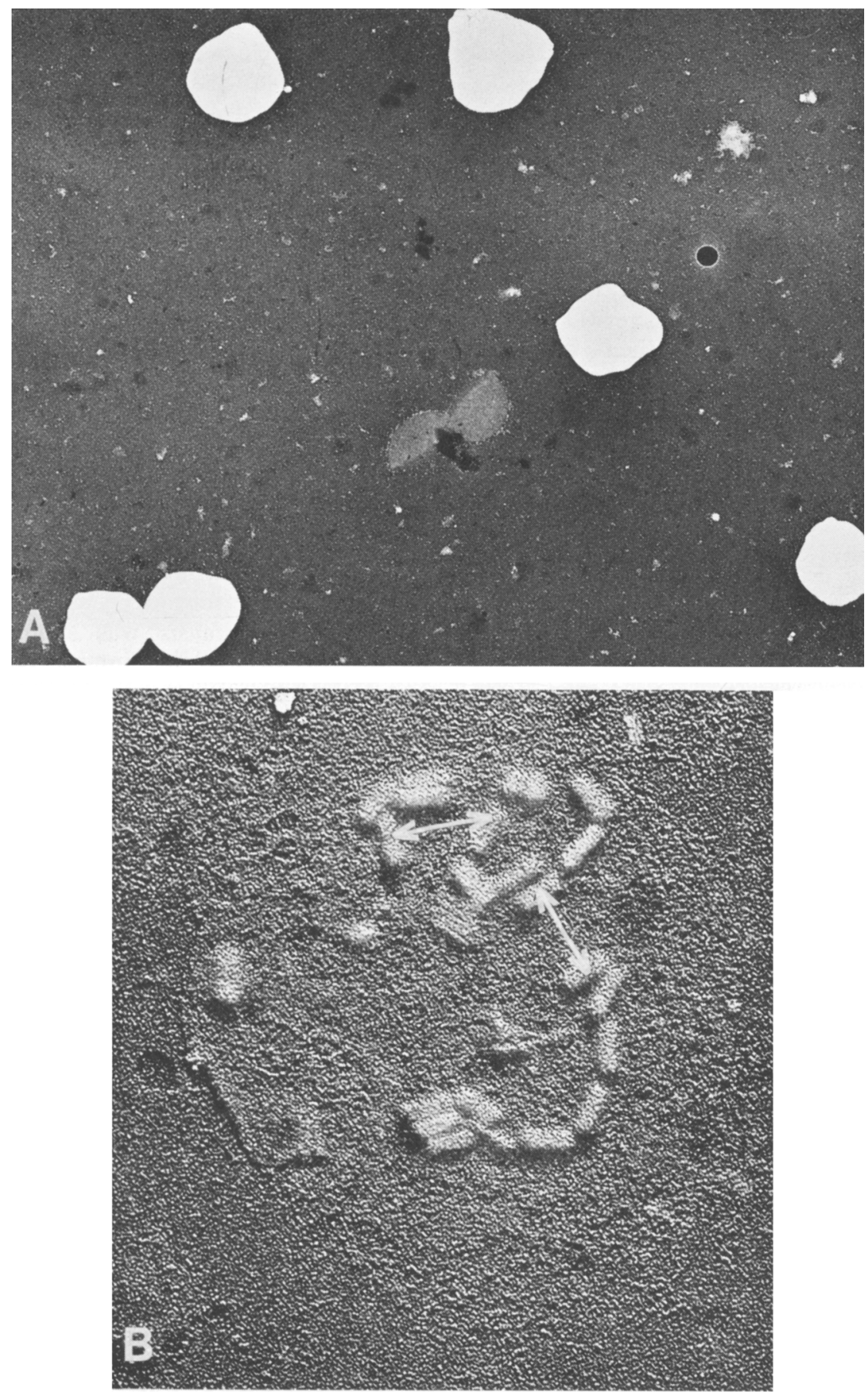

Fig. 29. Acc. No. 1961: Electron micrographs of a nuclear polyhedrosis virus from Bylesia nigricans. A: Polyhedra. Magnification $=10,500 \mathrm{X}$. B: Dissolved polyhedron showing included virus rods (arrows). Magnification $=19,000 \mathrm{X}$. 
imen was submitted by R. L. Rabb, Raleigh, North Carolina, on December 15, 1965. Diagnosis: Bacteriosis: mainly Pseudomonas aeruginosa.

\section{Syntomidae}

\section{Ceramidia muscicola (Ckll)}

2440-Several specimens were submitted by F. Lara E., Limon, Costa Rica, on July 28, 1965. Diagnosis: Mycosis caused by Aspergillus flavus and a secondary, saprophytic Penicillium sp.

2637-Eight specimens were received from F. Lara E. on June 21, 1966. Diagnosis: Bacteriosis caused by Streptococcus faecalis var. liquefaciens.

2753-Several more pupae and larvae were submitted by $F$. Lara $E$. on May 29, 1967. Diagnosis: Bacterioses caused by Bacillus cereus and Micrococcus conglomeratus.

\section{Tineidae}

\section{Nephantis serinopa (Meyrick)}

2098-A number of clothes moth larvae were submitted by U. Ekanayake, Lunuwila, Ceylon, on August 9, 1963. They had been treated with Bakthane L-39, a commercial Bacillus thuringiensis preparation. Diagnosis: Bacteriosis caused by Bacillus thuringiensis.

\section{Tineola biselliella (Hummel)}

2598-Several laboratory-reared larvae were submitted by $\mathrm{H}$. Laudani, Savannah, Georgia, on April 18, 1966. Diagnosis: Possible polyhedrosis virus in one larva.

\section{Tortricidae}

\section{Archips argirospilus (Walker)}

2991-One dead larva collected from red bud was submitted by D. Pinnock, Berkeley, Califormia, on May 19, 1971. Diagnosis: Virosis caused by a granulosis virus.
Choristoneura fumiferana (Clemens)

2277 - Several laboratory-reared specimens of the spruce budworm were submitted by G. Downing, Berkeley, California, on June 18, 1964. Rearing conditions were humid. Diagnosis: Mycosis caused by Beauveria bassiana.

2301-Some specimens were submitted by B. Barr, Berkeley, California, on July 22, 1964. The larvae were reared on an artificial diet in conditions of high humidity. Diagnosis: Bacteriosis caused by Serratia marcescens.

2451-Several spruce budworm larvae were submitted by G. Downing, Berkeley, California, on August 5, 1965. Diagnosis: Mycosis caused by Beauveria bassiana.

2635-Several larvae were received from R. L. Lyon on June 16, 1966. Diagnosis: Non-microbial. Mortality probably due to abnormally long diapause in cold storage.

2733-Several additional larvae were received from R. L. Lyon on March 6, 1967. Diagnoses: Virosis caused by a granulosis virus.

2738-Several larvae were submitted by C. Richmond, Berkeley, California, on April 17, 1967. Diagnosis: Virosis caused by a granulosis virus.

2756 - Several more larvae were submitted by C. Richmond on June 28, 1967. Diagnoses: Viroses caused by a nuclear polyhedrosis and a granulosis. Also Beauveria bassiana in one case. (Fig. 30.)

2814-R. L. Lyon, Berkeley, California, submitted several larvae of the spruce budworm on March 22, 1968. Diagnosis: Three showed no microbial etiology and one showed a microsporidian infection caused by Glugea fumiferanae.

2837-On June 4, 1968, R. L. Lyon submitted more spruce budworm larvae from his laboratory cultures. Diagnosis: Non-microbial. 


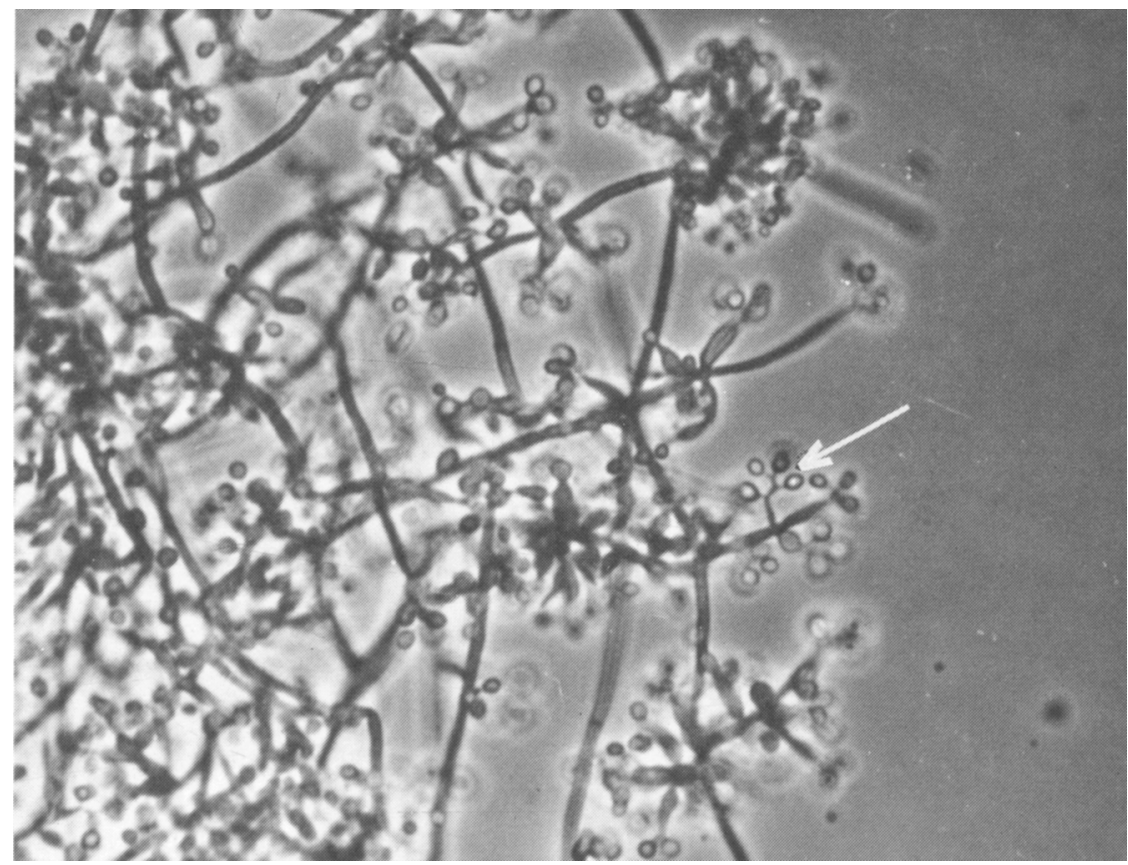

Fig. 30. Acc. No. 2756: Phase-contrast micrograph of a sporulating culture of Beauveria bassiana isolated from Choristoneura fumiferana. Note typical zigzag phialide (arrow). Magnification $=800 \mathrm{X}$.

2907-R. L. Lyon again submitted several larvae from his laboratory cultures on August 20, 1969. Diagnosis: Non-microbial.

2975-Several laboratory-reared pupae and larvae were submitted by $R$. L. Lyon, Berkeley, California, on December 12, 1970. Diagnosis: Non-microbial.

\section{Choristoneura occidentalis Freeman}

2762_On July 24, 1967, several specimens were submitted by C. Richmond, Berkeley, California. The specimens had been collected in the Bitterroot Mountains of Idaho. Diagnosis: Virosis caused by a nuclear polyhedrosis virus.

2870 - One larva from the laboratory culture of R. L. Lyon was submitted on November 6, 1968. Diagnosis: Mycosis caused by Aspergillus flavus.

2903-On July 23, 1969, two pupae and ten larvae were submitted by J. R. Milodragovich, Missoula, Montana. The specimens had been collected in the Blackfoot River drainage near Missoula. Diagnosis: Virosis caused by a nuclear polyhedrosis virus.

2918-Eggs and first instar larvae on an artificial medium were submitted by R. L. Lyon, Berkeley, California, on September 24, 1969. Diagnosis: Nonmicrobial.

\section{Choristoneura pinus Freeman}

2811-Four larvae of the jack pine budworm were submitted by $R$. L. Lyon, Berkeley, California, on February 28,1968 . The larvae were from field-collected stock reared in the laboratory. Diagnosis: Non-microbial.

\section{Pandemis heparana (Schiff)}

2368-These larvae were submitted by T. Edland, Vollebekk, Norway, on 
March 16, 1965. Diagnosis: Secondary bacteriosis caused by Streptococcus sp.

\section{Pandemis ribeana (Hübner)}

2367-Additional larvae were submitted by T. Edland on March 16, 1965. Diagnosis: Secondary bacteriosis caused by Aerobacter grp. C.

\section{Platynota rostrana (Walker)}

2378-These specimens were submitted by F. Lara E., Limon, Costa Rica. They were collected in La Estrella Valley on March 2, 1965. Diagnosis: Parasitism by a Chalcid wasp.

2441-Additional specimens were submitted by F. Lara E. on July 28, 1965. Diagnosis: Possible mycosis caused by Fusarium sp. with secondary invasion by acarid mites.

\section{Zygaenidae}

\section{Argyrotaenia velutinana (Walker)}

2767-Several larvae and pupae of the red banded leafroller were submitted by G. C. Rock, Raleigh, N.C., on August 14, 1967. Diagnosis: Non-microbial.

\section{Unidentified}

2029_-One specimen was submitted by R. C. Hunter, Buga, Valle, Colombia, on March 18, 1963. Diagnosis: Mycosis caused by Beauveria bassiana.

2207-Several larvae were submitted by D. McKay, St. Petersburg, Florida, on December 11, 1963. Diagnosis: Nonmicrobial.

2223-Some specimens were submitted by J. Powell, Berkeley, California, on January 31, 1964. Diagnosis: Nonmicrobial.

\section{(Possibly Cankerworm)}

2298_-One specimen was received from J. D. Stein, Fargo, North Dakota, on July 10, 1964. Diagnosis: Parasitism.
2304 -One specimen collected in Blodgett Forest, California, was submitted by R. Stark, Berkeley, California, on July 24, 1964. Diagnosis: Nonmicrobial; mechanical damage.

2392-Two larvae were collected at Kahua Ranch, Wiameakohala, Hawaii, by Y. Tanada on April 22, 1965. Diagnosis: Mycosis caused by Beauveria bassiana.

2411-A single larva was submitted by J. Hoy, Berkeley, California, on June 1, 1965. Diagnosis: Microsporidiosis possibly caused by a Nosema sp.

2444 - These specimens were submitted by F. Lara E., Limon, Costa Rica, on July 28,1965 . The extent of disease outbreak was over 90 per cent. Diagnosis: Primary etiology was obscured by the action of acarid mites.

2571-One dead specimen was submitted by J. Powell, Berkeley, California, on March 2, 1966. Diagnosis: Bacteriosis caused by Streptococcus faecalis with the secondary invaders Aerobacter sp. and Escherichia coli.

\section{Unidentified tip moth}

2899-Two larvae of a tip moth collected at Blodgett Forest, California, were submitted on July 1, 1969 by R. Stark, Berkeley, California. Diagnosis: Non-microbial.

\section{NEUROPTERA}

\section{Chrysopidae}

Chrysopa carnea Stephens

2425 - Several laboratory-reared specimens were submitted by $\mathrm{Mr}$. Tasan, Berkeley, California, on June 28, 1965. Diagnosis: Bacterioses caused by Streptococcus faecalis and Serratia marcescens.

2879-Several adults of this lacewing were submitted by $R$. Tassan, Albany, California, on January 24, 1969. Diagnosis: Microsporidiosis caused by Plistophora californica. 


\section{Chrysopa vittata Wesmael}

2765-One pupa was submitted by P. H. Thompson, New Brunswick, New Jersey, on July 31, 1967. Diagnosis: Bacteriosis caused by Bacillus cereus, with Aerobacter sp. as a secondary invader.

\section{Chrysopa zastrowi}

3029-Larvae of this lacewing were submitted by S. W. Broodryk, Pretoria, South Africa, on November 19, 1971. These were from cultures where mortality reached as high as 50 per cent. Diagnosis: Probably non-microbial, with secondary invasion in some by Bacillus cereus.

\section{Chrysopa sp.}

1926-_Several specimens were submitted by C. A. Toschi, Berkeley, California, on August 10, 1962. Diagnosis: Non-microbial.

2776-A few adults were submitted by K. Hagen, Berkeley, California, on October 4, 1967. Diagnosis: Bacteriosis caused by the potential pathogen Streptococcus faecalis var. liquefaciens.

\section{Meleoma cavifrons (Banks)}

1987-These specimens were submitted by C. A. Toschi, Berkeley, California, on December 7, 1962. They were field-collected and reared in the laboratory. Diagnosis: Non-microbial.

\section{Meleoma delicata Banks}

2563-Several dead specimens were submitted by C. A. Toschi, Berkeley, California, on February 9, 1966. Diagnosis: Secondary bacteriosis caused by Aerobacter sp. Cause of death was probably non-microbial.

\section{Hemerobiidae}

Unidentified (brown lacewing)

1927-One specimen collected at Sagehen Creek, California, was sub- mitted by C. A. Toschi, Berkeley, California, on August 10, 1962. Diagnosis: Parasitism.

\section{ORTHOPTERA}

\section{Acrididae}

Aealoplus turnbulli (Thomas)

1928-One specimen was submitted by $\mathrm{R}$. Pfadt, Laramie, Wyoming, on August 13, 1962. Diagnosis: Mycosis caused by Entomophthora grylli.

\section{Aularches miliaris (L.)}

1937 - Several laboratory-reared specimens were submitted by B. Meksongsee, Bangkok, Thailand, on September 4, 1962. Amoeba-like bodies were isolated from the body cavity, but infectivity tests were negative. Diagnosis: No definite pathogen found.

\section{Aulocara elliotti (Thomas)}

1910-Two specimens were submitted by R. Pfadt, Laramie, Wyoming, on July 30, 1962. Diagnosis: Non-microbial.

1929-Additional specimens were submitted by R. Pfadt on August 13, 1962. Diagnosis: Non-microbial.

\section{Chorthippus curtipennis (Harris)}

2955-Several specimens of this grasshopper were submitted by W. J. Loher, Berkeley, California, on July 10, 1970. The specimens were collected from San Bruno Mt., California, for experimentation. Diagnosis: Probably non-microbial.

\section{Dendrotettix quercus Pck.}

2800 -Several fungus cultures isolated from this grasshopper were received from $\mathrm{H}$. Coppel, Madison, Wisconsin, on January 22, 1968, with a request for their identification. Diagnosis: Non-sporulating Hyphomycete, a non-sporulating Dematiaceae; two species of Fusidium, two Ascomycete 
cultures near Thielavia and a Verticillium sp.

\section{Hieroglyphus banian (Fabricius)}

1936-On September 4, 1962, 10 grasshoppers were received from B. Meksongsee, Bangkok, Thailand. They had been collected from corn plants in the Lopburi Province of Thailand, where they were being killed by an epizootic. Diagnosis: Mycosis caused by Entomophthora grylli. This is a new host record for this fungus.

\section{Melanoplus bivittatus (Say)}

1902-Two adults were submitted by R. D. Frye, Fargo, North Dakota, on July 20, 1962. Diagnosis: Mycosis caused by Entomophthora grylli.

\section{Melanoplus occidentalis (Thomas)}

1912-A single specimen was submitted by R. Pfadt, Laramie, Wyoming, on July 30, 1962. Diagnosis: Non-microbial.

\section{Patanga succincta (L.)}

2185-Ten adult females from the Taklee District, Nakorn-Sawan Province, Thailand, were submitted by $\mathrm{B}$. Meksongsee, Bangkok, Thailand, on October 16, 1963. The disease appeared to be of epizootic proportions. Diagnosis: Mycosis caused by Entomophthora grylli.

\section{Schistocerca gregaria (Forsk.) and} S. vaga Brun.

2514-Several dead specimens of both species were submitted by $J$. Caraco, Berkeley, California, on November 18, 1965. All the specimens but one had been preserved. Examination of the ventilating system in the rearing facilities showed a direct connection to animal-rearing rooms where insecticides were routinely used. Moving the grasshopper cultures to other quarters solved the problem. Diagnosis: Toxicosis.

\section{Schistocerca gregaria (Forsk.)}

2754-Three adults were submitted by a locust entomologist from Bikaner (Rajasthan), India, on June 5, 1967. Diagnosis: Bacteriosis caused by Pseudomonas aeruginosa.

2986-Eight adult desert locusts were submitted by M. V. Venkatesh, Bikaner, India, on April 26, 1971. The locusts had been reared in the laboratory on cauliflower leaves and 50 per cent of the colony had become infected. Diagnosis: Bacteriosis caused by Bacillus cereus.

\section{Schistocerca obscura Brun.}

2912_Two adult grasshoppers were received from W. G. Genung, Belle Glade, Florida, on September 8, 1969. Diagnosis: Microsporidiosis caused by Nosema locustae.

\section{Grillidae}

\section{Acheta domesticus (L.)}

2183-Three adults were submitted by P. C. Stone, Columbia, Missouri, on October 8, 1963. Diagnosis: Bacteriosis caused by Bacillus cereus.

\section{Phasmatidae}

\section{Anchiale majalis}

2974 - Ten eggs of this walking stick were submitted by G. O. Bedford, Keravat, New Guinea. The eggs were mummified by a fungus. Diagnosis: Mycosis caused by Aspergillus flavus.

\section{Didymuria violescens (Leach)}

2719-Many eggs were received from J. L. Readshaw, Albury, New South Wales, Australia, on January 12, 1967. Diagnosis: Mycosis caused by Paecilomyces farinosis.

\section{Tettigoniidae}

Cordillacris occipitalis (Thomas)

1911_Four adults were submitted by R. E. Pfadt, Laramie, Wyoming, on July 30, 1962. Diagnosis: Non-microbial. 


\section{Tryxalidae}

Dichromorpha viridis Scud.

2923-On October 27, 1969, several adult grasshoppers were received from
W. G. Genung, Belle Glade, Florida. Diagnosis: Mycosis caused by Entomophthora grylli.

\section{Arachnida}

\section{ACARINA}

\section{Ixodidae}

\section{Amblyomma tuberculatum (Marx)}

2437-Two adult females of the gopher-tortoise tick from Junction City, Georgia, were submitted by J. C. Cooney, Auburn, Alabama, on July 26, 1965. Diagnosis: Non-microbial.

\section{Unidentified}

2533-Many dead specimens in a glass jar were submitted by D. McKay, St. Petersburg, Florida, on November 15, 1965. Diagnosis: Non-microbial.

\section{Tetranychidae}

Panonychus ulmi (Koch)

2856-Eggs and immatures of the European red mite were submitted by G. C. Rock, Raleigh, North Carolina, on August 27, 1968. They were collected at Jackson Springs, North Carolina, on August 8, 1968, from apple. Diagnosis: Non-microbial.

\section{Tetranychus cinnabarinus (Boisd.)}

2380-These specimens were submitted by H. N. Plaut, Haifa, Israel, on March 31, 1965. Diagnosis: Non-microbial.

2676-Numerous more specimens were received from $\mathrm{H}$. N. Plaut, Haifa, Israel, on August 19, 1966. Diagnosis: Mycosis caused by an Entomophthora sp.

2872-Several mites were received from H. N. Plaut, Haifa, Israel, on December 2, 1968. Diagnosis: Possible rickettsiosis. The submitted material was rather deteriorated, but electron microscope studies indicated a possible rickettsial infection.
Tetranychus cinnabarinus Boisduval \& T. urticae (Koch)

2968_-These mites were collected from bean plants near Haifa, Israel, and submitted by H. N. Plaut on September 21, 1970. The specimens were mixed in a single vial. Diagnosis: Mycosis caused by an Entomophthora sp.

Tetranychus pacificus McG.

2915-A few adults of the Pacific mite from grape leaves were submitted by M. Hoy, Reedley, California, on September 15, 1969. Diagnosis: Nonmicrobial.

\section{T'etranychus urticae (Koch)}

2386-A single mite was submitted by A. Ota, Berkeley, California, on April 9, 1965. The specimen was reared at a temperature of $86^{\circ} \mathrm{F}$ with continuous light. Diagnosis: Bacteriosis caused by Pseudomonas aeruginosa.

2802-Many adults of the two spotted red mite enclosed in a mat of fungus were submitted by T. Kono, Sacramento, California, on January 22, 1968, with a request for the identification of the fungus. Diagnosis: The fungus was identified as a Cladosporium sp. which had physically trapped the mites in a mass of mycelium, but examination showed no invasion of the mites by the fungus.

\section{Unidentified}

2176-Several fungal slides arıd some specimens were submitted by $\mathrm{A}$. L. Morehart, Newark, Delaware, on October 2, 1963. Diagnosis: Mycosis caused by Entomophthora sp. 


\section{Trombiculidae}

Vatacarus ipoides (Southcott)

2250 - Several laboratory-reared specimens were submitted by F. J. Radovsky, San Francisco, California, on April 14, 1964. Diagnosis: Probably rickettsia with a mycosis caused by Entomophthora sp. This is a new host and pathogen record.

\section{Unidentified}

1923-This specimen was submitted by D. E. Shaw, Papua, New Guinea, on August 8, 1962. Diagnosis: Mycosis caused by a Gibellula sp. near Gibellula leiopus.

3010-On August 13, 1971, several unidentified mites were submitted by C. M. Searle, Mazoe, Rhodesia. Diagnosis: Mycosis caused by a Hirsutella sp.
3025-Several mites were again submitted by C. M. Searle, Mazoe, Rhodesia, on November 8, 1971. Diagnosis: Mycosis caused by a fungus which was not viable and could not be identified.

\section{ARANEIDA}

\section{Unidentified spider}

1924 A single specimen on a leaf of Coffea arabica was submitted by J. J. H. Szent-Ivany; Konedobu, Papua, New Guinea, on August 9, 1962. Diagnosis: Mycosis caused by Gibellula aranearum. A new area record for this fungus. (Fig. 31.)

2924-Two adult spiders were submitted by F. Ennik, Berkeley, California, on November 5, 1969. Diagnosis: Non-microbial.

\section{Crustacea}

\section{ISOPODA}

\section{Armadillidiidae}

Armadillidium vulgara (Latreille)

2932-Several specimens of this sow bug were submitted by O. Paris, Berkeley, California, on December 30, 1969. Diagnosis: Mycosis caused by an $\boldsymbol{E} \boldsymbol{n}$ tomophthora $\mathrm{sp}$.

\section{CIRRIPEDIA}

\section{Family Unknown}

Elimnius modestus

2225-Four dead barnacles pre- served in alcohol were submitted by $\mathrm{J}$. T. Tomlinson, Kaikoura, New Zealand, on January 25, 1964. Diagnosis: infection by the algae Rhodophyceae bangiaceae and Neevea sp.

\section{DECAPODA}

Atyidae

Crayfish

2664-Many dead specimens from Robbins, California, were submitted by M. D. Miller, Davis, California, on August 2, 1966. Diagnosis: Non-microbial.

\section{Symphyla}

\section{CEPHALOSTIGMATA}

\section{Scutigerellidae}

\section{Scutigerella immaculata (Newport)}

1847-A fungus culture and stained whole mount of the garden symphylan were submitted by L. W. Getzin, Puyallup, Washington, on March 9, 1962. Diagnosis: Mycosis caused by Ento- mophthora coronata. A new host and area record for this fungus.

2259-An agar slant containing a fungus isolated from this garden symphylan was submitted by L. W. Getzin, Puyallup, Washington, on May 5, 1964. Diagnosis: Metarrhizium anisopliae. New record. 

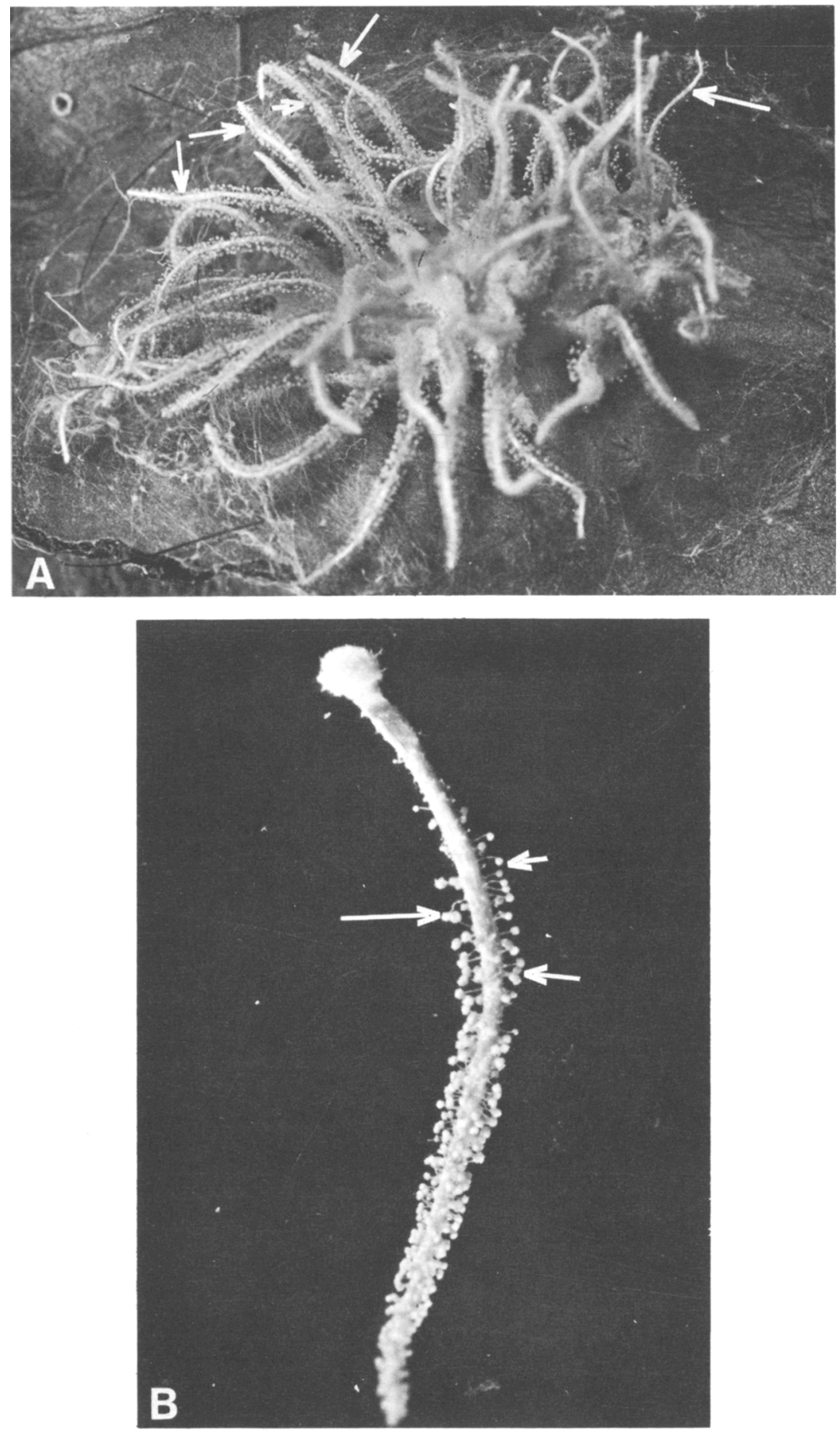

Fig. 31. Acc. No. 1924: Spider infected with Gibellula aranearum. A: Infected spider on leaf Note synnematia of the fungus (arrows). Magnification $=6 \mathrm{X}$. B: Single synnematium of the fungus showing sporophores with spore masses (arrows). Magnification $=20 \mathrm{X}$. 


\section{ANNELIDA}

\section{Oligochaeta}

\section{OPISTHOPORA}

\section{Lumbricidae}

\section{Lumbricus sp.}

1861-Several lots of earthworms were submitted by E. Long, Savannah, Tennessee, on March 11, 1962. Diagnosis: There was some evidence of a possible virosis, however, this was not definitely confirmed.

2010-These specimens from commercial worm beds were submitted by H. Stiegler, Humboldt, Tennessee, on February 8, 1963. Healthy worms were submitted and there seemed to be no mortality. Diagnosis: No disease found.

2020-These specimens were collected from commercial worm beds and submitted by E. Long, Savannah, Tennessee, on February 25, 1963. The specimens appeared to be healthy. Diagnosis: Inconclusive. Spherical particles, about 30 millimicrons in diameter, were found in electron microscope preparations from supposedly diseased worms. However, further examination showed the same particles present in healthy worms. No other possible pathogens were found.

\section{Unidentified earthworms}

2031-These specimens were collected from commercial worm beds and submitted by $\mathrm{H}$. Stiegler, Humboldt, Tennessee, on March 23, 1963. Diagnosis: Undetermined.

2041-These specimens were collected from commercial worm beds and submitted by H. V. Dunnegan, Kingsburg, California, on April 12, 1963. Diagnosis: Undetermined.
1940-These specimens from Kamuela, Hawaii, were submitted by $\mathrm{Y}$. Tanada, Berkeley, California, on September 10, 1962. Diagnosis: Cause of death unknown, but may have been due to the dry condition of the turf.

2787-Some earthworms were received from D. C. Knutson, Bloomfield, New Mexico, on November 20, 1967. Diagnosis: Non-microbial. Several rather common soil bacteria were isolated, including Aerobacter sp. Pseudomonas aeruginosa, Shigella paradysenteriae, Bacillus cereus, Salmonella paratyphi A, Corynebacterium sp., and Klebsiella pneumoniae.

\section{PLESIOPORA}

\section{Enchytraeidae}

Enchytraeus fragmentosus (Bell)

1864-Several specimens were submitted from both axenic and xenic cultures by E. C. Dougherty, Berkeley, California, on April 18, 1962. Diagnosis: Possible virosis.

1894-More laboratory-reared worms were submitted in axenic culture tubes by E. C. Dougherty on June 27, 1962. It was found that tumors would occur on the specimens when excessive moisture was present on the tubes. Numerous bodies resembling immature worms or vermiform bodies were present. Diagnosis: No microbial cause of death was determined.

1898-Additional specimens from axenic cultures were submitted by $\mathbf{E}$. C. Dougherty on July 19, 1962. Diagnosis: Examination revealed virus-like particles, but identification beyond this was not possible. 


\section{MICROORGANISMS}

\section{Microbial Cultures}

2914-Eight microbial cultures on agar slants were received on September 9, 1969, from G. McClure, Yonkers, New York. The microorganisms were from a study to identify bacteria and fungi associated with pesticide degradation. Those submitted had been isolated from a medium containing iso-
propyl-N-phenyl carbamate as the sole source of carbon. Diagnosis: The microorganisms were identified as follows: Fusarium solani var. minus Pseudomonas aeruginosa four species of Corynebacterium, Penicillium stoloniferum, Alcaligenes sp., Salmonella sp. nr. paratyphi; and an Actinomycete.

\section{Schizomycetes}

\section{EUBACTERIALES}

\section{Bacillaceae}

Bacillus thuringiensis Berliner 2391-Contaminated cultures of Bacillus thuringiensis were submitted by H. D. Burges, Slough, Bucks, England, on April 19, 1965. Diagnosis: Bacterium identified as Pseudomonas aeruginosa.

\section{PSEUDOMONADLES}

\section{Pseudomonadaceae}

Pseudomonas aeruginosa (Migula)

2656-A wet mount of this bacterium was submitted by P. Messenger, Berkeley, California, on July 27, 1966. Diagnosis: This bacterium is not considered a primary insect pathogen, but is placed in the category of a potential pathogen. It has no invasive powers of its own, but readily causes a fatal septicemia when it gains entrance into the hemocoel.

\section{CHORDATA CRANIATA}

\section{Aveas}

\section{NEOGNATHA}

\section{Chicken Egg}

2942-One chicken egg containing a nematode was submitted by A. Ana- walt, San Anselmo, California, on March 23, 1970, with a request for the identification of the nematode. Diagnosis: Ascaridia galli.

\section{LITERATURE CITED}

Brewer, Jo and G. M. Thomas

1966. Causes of death encountered during rearing of Danaus plexippus (Danaidae). J. Lepid. Soc. 20 (4) :235-38.

Dahlsten, D. L. and G. M. Thomas

1969. A nucleopolyhedrosis virus in populations of the Douglas fir tussock moth, Hemerocampa pseudotsugata, in California. J. Invert. Pathol. 13 (2) :264-71.

Steinhaus, E. A.

1951. Report on diagnoses of diseased insects 1944-1950. Hilgardia 20 (22):629-78.

Steinhaus, E. A. and G. A. Marsh

1962. Report of diagnoses of diseased insects 1951-1961. Hilgardia 33 (9) :349-90.

Thomas, G. M. and A. Luce

1972. An epizootic of chalk brood, Ascosphaera apis (Massen ex Claussen) Olive and Spiltoir in the honeybee, Apis mellifera L. in California, American Bee J. 112 (3) :88-90. 


\section{INDEX TO HOST INSECTS}

\section{(Listed According to Genus and Species)}

A.

Abcbaca, 335

aberrans, 299-300

abictis, 298

Abraxas, 310

Acantholyda, 301

Achaea, 317, 318

Acheta, 345

acrea, 304

Acrobasis, 335

Acrothosiphon, 290

acuta, 294

Adclges, 289

Adclphocoris, 288

Acaloplus, 344

Aedes, 281-282

aegypti, 281

Aeneolamia, 291

affinis affinis, 301

agrestis, 319

Agrotis, 319

ajax, 330

Alabama, 319

albilinia, 324

albovariegatus, 270

Aleurodes, 290

Alsophila, 310

alsophila, 301

Altermetoponia, 287

Amblyomma, 346

americana, 285

americana americana, 296

americanum, 313

amphimome, 337

amygdali, 298-299

anagasta, 331

anaphae, 276

Anastrepha, 287

Anchiale, 345

androgea, 323

angelica, 295

Anomala, 272

Anomis, 310

antaeus, 274

Antarctia, 304

Antheraea, 336-337

Anthocoris, 287

Anticarsia, 319

antiopa, 328

antiqua, 284

anurus, 300

Aonidiella, 293

apache, 292, 293

Aphis, 290

apicaulis, 314-315

Apis, 295-296, 297

appressa, 264
Aramminchus, 266

archippus, 328

Archips, 341

argentata, 305

argillacea, 319

argirospilus, 341

Argyresthia, 312

Argyrotaenia, 343

Armadillidium, 347

armigera, 320

Arrhcnodes, 263

asperipennis, 270

Aspidiotus, 294

assamensis, 336

astictopus, 280-281

Athalia, 301

Atta, 299

Attacus, 337

Aularches, 344

Aulocara, 344

aurantii, 293

auricularia, 279

australis, 289

Automeris, 337

autumnata, 311

aversa, 323

B

Ballana, 292

banian, 345

barodensis, 290

Bathycoelia, 289

Bathyplectus, 300

beckii, 294

belladonna, 334

Bembecia, 303

bicincta, 291

biselliella, 341

Biston, 310

bivittatus, 345

Blatella, 262

Bombyx, 307

Bothynus, 272-273

bouliana, 330

bozemani, 302

Bracon, 296

brassicae, 290, 334

Bremesia, 290

brevicomis, 275

Brevicoryne, 290

brumata, 311-312

brunneipennis, 266

Buzura, 310

C

Cactoblastis, 331

cactorum, 331

calcitrans, 284

californica, 328 californicum, 300

Caligo, 328

calligraphus, 275

Calophasia, 319

Calosoma, 263

campbelli, 279-280

canicularis, 283

capitata, 286

cardinalis, 289

cardui, 328

carduorum, 272

carnea, 343

Carpocapsa, 329-330

castaneum, 276-277

Castnea, 307

cavifrons, 344

cecropia, 337-338

Cecyropa, 266

Cephus, 296

ceramicus, 307

Ceramidia, 341

Ceratitus, 286

Chaoborus, 280-281

Chermes, 291-292

Chilo, 307-308

Chilotraea, 335

Chironomus, 281

Chlorina, 292

Choristoneura, 341-342

Chorizagrotis, 319

Chorthippus, 344

Chrysopa, 343-344

Chrysops, 285

Cimbex, 296

cinctus, 296

cinerea, 272

cinnabarinus, 346

cinticollis, 279

citrifolii, 290

clavifer, 288

Coccus, 292-293

Coelocnemis, 276

Colaspis, 272

Coleophora, 307

Colias, 333-334

Colladonus, 292

Coloradia, 337

completa, 286

concinna, 328

concolor, 296

confusum, 277, 278

constricta, 313

concolvuli, 339

Copris, 273

Cordillacris, 345

cornutus, 273

Cosmopolites, 266 
Cossonus, 266

Costelytra, 273

craccivora, 290

cretica, 325

cribicollis, 266

Culex, 282-283

Culicida, 283

cunea, 305, 306

cupressella, 312

curtipennis, 344

Cyclocephala, 273

Cylas, 266

Cylinarocopturus, 266

Cymatodera, 264

cynthia, 339

D

Dacus, 286

Danaus, 308-309

Darna, 314

Dasychira, 315-316

Delias, 334

delicata, 344

Deltacephalus, 292

Dendroctonus, 275

Dendrolimus, 313

Dendrotettix, 344-345

Desmia, 335

destructor, 277, 294

devastans, 292

Diabrotica, 264-265

Diacrisia, 304

Diadegma, 300

Dialeurodes, 290

Diatraea, 308

diatraeae, 286

Diceroprocta, 292, 293

Dichromorpha, 346

Didymuria, 345

diemenalis, 336

Dimmockia, 298

Dinocoris, 289

Diprion, 296, 298

Dirhodum, 290-291

Dirphia, 337

disstria, 313

Domestica, 284

domesticus, 345

dorsalis, 286

Draeculacephala, 294

Drosophila, 283

dubius, 264

Dysdercus, 289

$\mathbf{E}$

eatoni, 266

Ecpantheria, 304

Eligma, 319-320

Elimnius, 347

clliotti, 344

Empoasca, 292

Enchytraeus, 349

Ennomus, 310

Entomobrya, 278-279
Ephestia, 331

Epicauta, 272

Epirrita, 311

equestris, 280

Eremobothynus, 273

erichsonii, 302

Estigmene, 304-305

Ethmia, 309

Euborellia, 279

eucalypti, 336

Euproctis, 316

Eupsilis, 320

eurilochus, 328

euryalis, 338

Eurytoma, 298-299

Euxoa, 320

exempta, 322, 325

exigua, 322, 325

exitiosa, 304

expansa, 272

$\mathbf{F}$

Fannia, 283

fascifrons, 292

Fenusa, 302

figulilella, 331

fiscellaria lugubrosa, 311

flavipes, 303

florilega, 279

Forficula, 279

formicalius, 266

fragmentosus, 349

frontalis, 275

frugiperda, 322, 325

fugitivus, 300

fumiferana, 341-342

funeralis, 335

furcifera, 292, 293

G

Galleria, 309

gemmatalis, 319

germanica, 262

gibbosus, 272-273

glabra, 281

glabrum, 270

Glossinia, 283

godmani, 269

Gonometa, 313

gossypiella, 309

gossypii, 290

graminivora, 286

Graphognathus, 266

Grapholitha, 330

gregaria, 345

grossulariata, 310

$\mathbf{H}$

Haematobia, 283-284

Halisidota, 305

Haltica, 272

Haplodiplosis, 280

hebetor, 296

helichrysi, 290

Heliothis, 320-322
Helopeltis, 288

Hemerocampa, 316

Hemileuca, 337

hemipterus, 268

heparana, 342

Herculia, 335-336

Herse, 339

Heterogomphus, 273

Hieroglyphus, 345

Holotrichia, 273

Hyalophora, 337-339

Hylemya, 279

Hylemyia, 284

Hylesia, 339, 340

Hуреra, 266-267

Hyperaspis, 265

Hyphantria, 305, 306

hypochlora, 272

Hypogaster, 300

immaculata, 347

incertulus, 336

incertus, 273, 298

includens, 324, 327

inclusum, 270-271

incomptus, 268-269

infuscatella, 335

innotata, 336

Inopus, 287

intermedius, 289

interpunctella, 333

ipoides, 347

Ips, 275

irritans, 283-284

isabella, 305

Isia, 305

$\mathbf{J}$

jacobaea, 328

janata, 317, 318

javanica, 298

$\mathbf{K}$

kasmirika, 336

kirbyi, 335

kuhniella, 331

$\mathbf{L}$

laevigata, 263, 264

laisalis, 336

Lambdina, 311

Laphygma, 322

lareynii, 268

Lasioglossus, 299-300

lateralis, 265

latiuscula, 322

lecontei, 298

lepida, 314

Lepidosaphes, 294

lesbia, 333-334

Lespesia, 286

Leucania, 322

Leucophaea, 263

leucophthalmus, 291

leucostoma, 279 
libocedrus, 301

licoides, 307

Ligyocoris, $287-288$

Limenitis, 328

lincolaris, 288

lineolatus, 288

Lissorhopturus, 267-268

littoralis, 325-326

litura, 323-324

Lixophaga, 286

lugens, 293

Lumbricus, 349

lunata, 316

lunula, 319

lutcomaculata, 301

Lygus, 288

$\mathbf{M}$

Macrocentrus, 296

Macrosiphum, 290

Macrosteles, 292

maculata, 302

maculiventris, 289

madens, 277-278

maderae, 263

Magicicada, 292

maidia, 291

maia, 337

mainensis, 302

majalis, 345

Malacosoma, 313

Manduca, 339

margaritosa, 323

marginata, 303, 310

mauritia ssp.

acconyctoides, 326

Megacyllene, 263

Megachile, 300-301

melanogaster, 283

melanoleuca, 289

melanopa, 265

Melanoplus, 345

Melanostoma, 285

Meleoma, 344

mellifera, 295-296, 297

mellonella, 309

merkeli, 298

Mesohomotoma, 294, 295

Metamasius, 268

Metisa, 335

Metopolophium, 290-291

mexicanaria, 312

Microlarinus, 268

miliaris, 344

milleri, 310

Mineola, 336

minutus, 263

mitocera, 312

modesta, 339

modestus, 347

molesta, 300, 330

molitor, 276

Moneilema, 263-264
Monochamus, 264

montanus, 292

mori, 307

morosa, 336

morsitans, 283

musca, 284

muscicola, 341

mylitta, 336-337

myopaeformis, 284

Mythimna, 322

Myzus, 291

$\mathbf{N}$

Nabis, 288

Nacoleia, 336

narcissus, 319-320

Nechryopus, 263

Nemocestes, 268-269

N cocurupira, 279-280

Neodiprion, 298

Nephantis, 341

Nephotettix, 294

Nepytia, 311

ni, 326-327

nigra, 293

nigricans, 339,340

nigrivitta, 335-336

Nilaparvata, 293

Nipaecoccus, 292

nitidus, 264

njalensis, 292

nokomis, 328

Nomia, 300

notatus, 264

nubilalis, 336

Nymphalis, 328

nysa, 313

obliqua, 304

obscura, 345

occidentalis, 342,345

occipitalis, 345

ochrogaster, 320

octasema, 336

octomaculatus, 285

Oiketychus, 335

oleae, 286, 293

olivaceus, 301

oliviae, 337

Oncopera, 312

operculella, 309, 310

Operophthera, 311-312

Ophiomyia, 279

Ophyra, 279

Opius, 296

Oporinia, 311

Orgiya, 316-317, 318

orizaba, 339

ornithogalli, 324

orthogonia, 319

oryzophilus, 267-268

Ostrinia, 336

Otiorynchus, 266
Oulema, 265

ovipennis, 264

Oxycanus, 312

$\mathbf{P}$

Pachyspinx, 339

pacificus, 346

Pagria, 265

Paleacrita, 312

Pandemis, 342-343

pandora, 337

Panonychus, 346

Pantomorus, 269

Papilio, 330

Papaipema, 322

Paracimex, 287, 288

Paramyelois, 331-333

Parasa, 314

Parategeticula, 313

parthenica, 307

pasadenana, 330

Patanga, 345

pauson, 273

pechumani, 290

Pectinophora, 309

Pegomyia, 284

Pentalitomastix, 298

Pcponapis, 295

Peponatis, 295

Perga, 301

Pericoptes, 273

Peridroma, 323

Periplaneta, 263

pernyi, 336

Persectania, 323

persicae, 291

Phaeoura, 312

phantasmaria, 311

Phenacaspis, 294

Philaenus, 291

philoxiphaga, 320

Phlebotomus, 285

Phthorimaea, 309-310

Phymatodes, 264

picea, 291-292

piceae, 289

Pieris, 334

pinifoliae, 294

piniphilus, 266

pinus, 342

pipiens fatigans, 282

pisi, 290

Pissodes, 269-270

pisum, 290

plagiata, 315-316, 326

plana, 335

platura, 279

Platynota, 343

plethoricus, 298

plexippus, 308-309

Plodia, 333

plumosus, 281

Podisus, 289 
Polistes, 301

polistiformis, 304

polistiformis

polistiformis, 304

pollinifera, 313

polyphemus, 337

Polyphylla, 273-274

polyxenes asterius, 330

pometaria, 310

pomonella, 329-330

Pontania, 302

postica, 266-267, 291

pratensis, 335

Prionoxystus, 307

Pristophora, 302

procer, 301

Prodenia, 323-324

Profenusa, 302

Prosapia, 291

Protolevcania, 324

Protoparce, 339

pruinosa, 295

proxima, 301

Pseudaletia, 324-325

Pseudococcus, 292

Pseudoplusia, 327

Pseudotsugata, 316-317

Psila, 294

Psilopholis, 272

punctata, 276

pupurifascia, 322

Pyemotes, 299

$\mathbf{Q}$

$$
\text { pyralis, } 325
$$

quaylii, 286

quercus, 344-345

quinquemaculata, 339

quinquefasciatus, 282-283

$\mathbf{R}$

rapae, 334

Recurvaria, 310

refinasus, 270

Reticulotermes, 303

Rhabdoscelus, 270

Rhagoletis, 286

Rhopalosiphum, 291

Rhyacionia, 330

Rhyssa, 301

ribeana, 343

ricini, 337

robiniae, 263, 307

rosae, 294

rostrana, 343

Rothschildia, 339

rotundata, 300-301

rubiceps, 287

rubida, 289

rubra, 339

s

Sacadodes, 325

saccharalis, 308

sacchari, 294 saevissima, 299

saevissima richteri, 299

Saissetia, 293

Samia, 339

sanguinarius, 285

Sanninoidea, 304

savagei, 263

satelitia, 320

Sceliodes, 336

Schistocerca, 345

Schizura, 328

scitula, 304

scitulella, 336

Scopeuma, 281

Scolia, 301

Scolypopa, 289

Scolytus, 275-276

Scutigerella, 347

Semiothisa, 312

seneciella, 284

separata, 324

septendecim, 292

sequoiae, 304

Sericesthis, 274

seriventris, 289

serinopa, 341

Sesamia, 325

sexmaculata, 312

sexta, 339

Sibine, 314-315

signata, 265

signaticollis, 273

similis, 296, 298

Siphanta, 294

Smicronyx, 270

sobrius, 287-288

Sogata, 292

Sogatella, 293

Solenopsis, 299

sollicitans, 282

sordidus, 266

spectabilis, 313

Speyeria, 328

Sphecodegastra, 299-300

Spodoptera, 325-326

spumarius, 291

stercorarium, 281

stimulea, 315

Stenopelmus, 270

Stomoxys, 284

Strategus, 274

subsignarius, 310

subsylvella, 335

subulifera, 310

succincta, 345

suspensa, 287

Syntexis, 301

Syrphus, 285

$\mathbf{T}$

tabaci, 290

Tabanus, 285-286

tarsalis, 283
Telenomus, 301

Telingana, 294

temnochila, 272

Tenebrio, 276

terminalis, 269-270

tessmanni, 294, 295

Tetanops, 284

Tetranychus, 346

Tetrastichus, 298

texana, 299

thalissma, 289

Thanasimus, 264

Thannosphecia, 304

Thaumatomyia, 281

Therioaphis, 291

Therion, 300

Tineola, 341

Tipula, 286-287

Tiracola, 326

togoi, 282

torvus, 285

Trabala, 314

Tragidion, 264

transitella, 331-333

Trialeurodes, 290

Triatoma, 289

Tribolium, 276-278

Trichoplusia, 326-327

tricolorella, 335

trifolii, 291

trima, 314

Trionymus, 294

Trogoderma, 270-271

truncatus, 273

Tryporyza, 336

tuberculatum, 346

turbata, 317, 318

turnbulli, 344

Tyria, 328

$\mathbf{U}$

ulmi, 302, 346

undecimpunctata

howardi, 264-265

unipuncta, 324-325

unostrigata, 278-279

urticae, 346

$\mathbf{v}$

vaga, 345

Vanessa, 328-329

varipes, 294

vastator, 292

Vatacarus, 347

velutinana, 343

venosata, 308

ventralis, 275-276

ventricosus, 299

vernata, 312

Vespamima, 304

Vespula, 302

vestita, 272

vetusta, 316

vexans, 282 
vexator occidentis, 285

violescens, 345

virescens, 320

virescens ssp.

chlorodia, 272

virginensis, 328-329

viridis, 346

vishnu, 314

Vitacea, 304

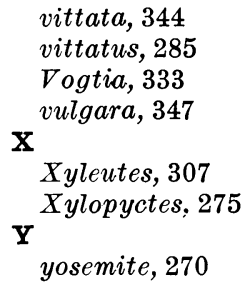

yosemite, 270

$\mathbf{z}^{\text {yps }}$

ypsilon, 319

zastrowi, 344

$z e a, 321$

zealandica, 273

Zelus, 288-289

zonellus, 307-308

zozana, 330

\title{
INDEX TO MICROORGANISMS AND OTHER MICROBIOTA
}

\author{
A \\ (Listed According to Genus)
}

Adelina sp., 270, 271, 280, 281

Adelina tribolii Bhatia, 277, 278

Aerobacter sp., 266, 267, 268, 275, 276, 280, 285, 286, 291, 303, 308, 309, 322, 323, $328,334,343,344,349$

Aerobacter aerogenes (Kruse) Beijerinck, 265, 322, 324

Aerobacter cloacae (Jordan) Bergey et al., 286, 329

Aerobacter liquefaciens Grimes \& Hennerty, 296, 300, 323, 328

Alcaligenes sp., 275, 285, 350

Alcaligenes faecalis Castellani \& Chalmers, 275, 307, 332

Alternaria sp., 294, 335

Ascaridia galli, 350

Aschersonia aleyrodis Webber, 290

Aschersonia goldiana Sacs. \& Ellis, 290

Aschersonia placenta Berkeley \& Broome, 290

Ascosphaera alvei (Betts) Olive \& Spiltoir, 303

Ascosphaera apis (Massen ex Claussen) Olive \& Spiltoir, 296, 297, 301

Aspergillus sp., 265, 290

Aspergillus delacroixii (Sacchardo) Thom \& Church, 300

Aspergillus effusus Tiraboschi, 337

Aspergillus flavus Link, 262, 264, 275, 276, 284, 286, 287, 288, 296, 299, 304, 310, $321,322,323,324,327,335,337,341,342,345$

Aspergillus flavus-oryzae grp., 337

Aspergillus fumigatus Fresenius, 300

Aspergillus glaucus Link, 300

Aspergillus lutescens Bainer, 337

Aspergillus niger van Tieghem., 278, 300, 316, 327, 337

Aspergillus ochracerus Wilhelm, 264, 265, 279, 301, 310, 327, 337

Aspergillus parasiticus Speare, 294, 337

Aspergillus sclerotium Huber, 310

Aspergillus sulphureus (Fresenius) Thom, 300

\section{B}

Bacillus sp., 335

Bacillus alvei Cheshire \& Cheyne, 296

Bacillus cereus Frankland \& Frankland, 264, 267, 272, 273, 274, 284, 285, 287, 288, $292,296,300,308,310,314,316,317,322$, $323,325,328,331,335,337,338,339,341$, $344,345,349$ 
Bacillus cereus var. mycoides (Flugge) Smith et al., 283, 284, 300, 317, 319, 323,

Bacillus entomocidus Heimpel \& Angus, 331, 332 328,332

Bacillus finitimus Heimpel \& Angus, 324

Bacillus megaterium deBary, 263, 294, 328, 329

Bacillus thuringiensis Berliner, 262, 286, 329, 331, 333, 335, 339, 341, 350

Bacillus thuringiensis var. entomocidus Heimpel \& Angus, 331, 332

Bacillus thuringiensis var. thuringiensis Berliner, 263, 275, 289, 309, 313, 317, 331,

Bactrodesmiella sp., 268

332

Beauveria bassiana (Balsamo) Vuillemin, 263, 264, 265, 266, 267, 268, 269, 270, $272,273,279,287,288,292,296 ; 299$, 301, 302, 303, 304, 307, 311, 320, 321, $322,323,327,329,330,335,336,337$, $341,342,343$

Beauveria tenella (Delacroix) Siemaszko, 265

Bergoldiavirus Group (Granulosis Virus), 305, 306, 308, 309, 310, 312, 314, 317, $318,320,321,322,323,324,325,329$, $333,336,341$

Birdiavirus Group (Nuclear Polyhedrosis Virus), 298

Borrelinavirus Group (Nuclear Polyhedrosis Virus), 305, 306, 310, 311, 312, 313, $315,316,317,318,320,321$, $323,324,325,326,327,328$,

C $333,337,339,340,341,342$

Candida sorbosa, 283

Cephalosporium sp., 289, 292

Chalaropsis sp., 267

Citrobacter, 263

Cladosporium sp., 290, 294, 303, 346

Clostridium sp., 283, 301

Clostridium saprogenes (Salus) McClung \& McCoy, 339

Cordyceps sp., 273, 274, 275, 304, 327

Cordyceps elosgata Petch, 304

Cordyceps melolonthae (Tulane) Saccardo, 275

Cordyceps ravenellii Berkeley, 275

Cordyceps sobolifera (Hill) Berkeley \& Broome, 292

Corynebacterium sp., 349, 350

Cytoplasmic Polyhedrosis Virus (Smithiavirus Group), 298, 311, 312, 313, 331, 335

$\mathbf{E}$

Entomophthora sp., 266, 279, 284, 286, 290, 291, 292, 293, 294, 295, 298, 304, 313, $316,322,323,324,327,346,347$

Entomophthora aphidis Hoffman, 290, 291, 292, 327

Entomophthora apiculata (Thaxter) M. Gustafs., 286

Entomophthora aulicae Reichardt, 307

Entomophthora coronata (Constantin) Kevorkian, 347

Entomophthora dipterigena (Thaxter) M. Gustafs., 284

Entomophthora echinospora (Thaxter) M. Gustafs., 287

Entomophthora erupta' Dustan, 288

Entomophthora forficulae Giard, 279 
Entomophthora grylli Fresenius, 304, 337, 344, 345, 346

Entomophthora megasperma (Cohn) Thaxter, 326

Entomophthora muscae (Cohn) Fresenius, 279, 281, 283, 284, 285

Entomophthora sphaerosperma Fresenius, 279, 285, 322, 325

Entomophthora thaxteriana (Petch) Hall \& Bell, 291

Erwinia sp., 263, 289, 292, 322

Escherichia coli (Migula) Castellani \& Chalmers, 307, 309, 343

Escherichia freundii (Braak) Yale, 273

\section{$\mathbf{F}$}

Farinocystis tribolii Weiser, 276, 277

Flavobacterium sp., 337, 339

Flavobacterium rhenanum (Migula) Bergy et al., 326

Fumago sp., 286

Fusarium sp., 264, 279, 286, 289, 290, 292, 293, 294, 295, 296, 298, 300, 302, 312, 313, $330,336,343$

Fusarium episphaeria (Tode) Snyder \& Hansen form coccophila (Desmazieres) Snyder \& Hansen, 294

Fusarium solani var. minus Wollenweber, 350

Fusidium sp., 309, 328, 344

G

Gibellula sp., 347

Gibellula aranearum (Schweinitz) Sydow, 347, 348

Gibellula leiopus (Vuillemin) Mains, 347

Gliocladium sp., 301

Glugea fumiferanae (Thomson) Weiser, 341

Glugea pyraustae (Paillot) Weiser, 336

Gordius sp., 278

Granulosis Virus (Bergoldiavirus Group), 305, 306, 308, 309, 310, 312, 314, 317, $318,320,321,322,323,324,325,329$,

\section{$\mathbf{H}$} $333,336,341$

Haffnia sp., 263, 289

Hirsutella sp., 322, 347

Hirsutella barberi (Giard) Petch, 308

Hirsutella citriformis Speare, 289, 293, 294

Hirsutella saussurei (Cooke) Speare, 301

Hormodendrum sp., 335

I

Isaria sp., 275, 294, 303

$\mathbf{K}$

Klebsiella pneumoniae (Schroeter) Trevisan, 349

$\mathbf{L}$

Leidynema sp., 263

$\mathbf{M}$

Masoniella sp., 322

Massospora cicadina Peck, 292

Mattesia sp., 332 
Mattesia dispora Naville, 270, 296, 298, 332, 333, 335

Metarrhizium anisopliae (Metchnikoff) Sorokin, 263, 266, 270, 272, 273, 284, 287, $295,299,301,304,312,323,325$, $335,336,347$

Metarrhizium brunneum Petch, 273, 289

Micrococcus sp., 292, 295, 300

Micrococcus conglomeratus Migula, 341

Monocillium sp., 296

Monosporium sp., 302

Mucor sp., 279, 286, 291

Myiophagus ucrainicus (Wize), 294

$\mathbf{N}$

Neevea sp., 347

Neoaplectana sp., 269, 329

Neoaplectana carpocapsae, 329

Neurospora sp., 294

Nosema sp., 264, 265, 276, 305, 309, 319, 321, 323, 328, 330, 332, 333, 336, 338, 343

Nosema bombycis Nägeli, 336

Nosema coliadis Jauch \& Jauch, 334

Nosema destructor Steinhaus \& Hughes, 332

Nosema locustae Canning, 345

Nosema polyvora Blunck, 333

Nosema whitei Weiser, 276, 277, 278

Nuclear Polyhedrosis Virus (Borrelinavirus Group \& Birdiavirus Group), 298, $305,306,310,311,312,313,315,316,317,318,320$, $321,323,324,325,326,327,328,333,337,339,340$, 341,342

$\mathbf{0}$

Oedocephalum sp., 276

$\mathbf{P}$

Paecilomyces sp., 267

Paecilomyces cossus (Port. \& Sart.) Brown \& Smith, 307

Paecilomyces fumoso-roseus (Wize) Brown \& Smith, 282

Paecilomyces (Spicaria) farinosus (Dicks. ex. Fr.) Brown \& Smith, 279, 293, 302, $304,305,310,312,345$

Panagrolaimus sp., 322

Penicillium sp., 264, 287, 294, 301, 302, 303, 308, 310, 313, 316, 319, 321, 327, 335, 339,341

Penicillium stoloniferum Thom., 350

Peymotes ventricosus (Newport), 299

Pichia membranaefaciens, 283

Plistophora californica Steinhaus \& Hughes, 343

Proteus sp., 265, 285, 292, 296, 309, 315, 322, 339

Proteus morganii (Winslow et al.) Rauss, 285

Pseudomonas sp., 285, 289, 309

Pseudomonas aeruginosa (Schroeter) Migula, 263, 265, 266, 275, 280, 283, 285, 286, 287, 290, 294, 301, 308, 312, 320, 322, 323, 325, 332, 336, $338,339,341,345,346,349,350$ 


\section{$\mathbf{R}$}

Rhizopus sp., 339

Rhizopus nigricans Ehrenberg, 264, 265, 276, 279, 298, 300, 302, 303, 313

Rhodophyceae bangiaceae, 347

\section{$\mathbf{S}$}

Sacbrood virus, 296

Salmonella sp., 350

Salmonella paratyphi A (Kayser) Castellani \& Chalmers, 349, 350

Saprolegnia sp., 280

Scopulariopsis sp., 337

Sericesthis Iridescent Virus (SIV), 274

Serratia marcescens Bizio, 263, 265, 266, 273, 274, 285, 288, 301, 307, 308, 309, 315,

Shigella sp., 309 $320,321,322,334,335,336,341,343$

Shigella paradysenteriae Weldin, 349

Smithiavirus Group (Cytoplasmic Polyhedrosis Virus), 298, 311, 312, 313, 331, 335

Sphaerostilbe sp., 293

Spicaria sp., 267, 322

Spicaria (Paecilomyces) farinosa (Dicks. ex. Fr.) Vuillemin, 279, 293, 302, 304, $305,310,312,345$

Spicaria rileyi (Farlow) Charles, 287, 312, 319, 320, 321, 322, 324, 325, 326, 327

Spirillum sp., 309

Stemonitis sp., 279

Streptococcus sp., 266, 267, 301, 304, 307, 309, 314, 319, 322, 323, 324, 325, 327, 335, 339,343

Streptococcus apis Maassen, 296

Streptococcus faecalis Andrewes \& Horder, 263, 267, 268, 276, 277, 278, 287, 296, $300,304,307,308,309,313,315,321$, 322 , 323, 324, 325, 328, 330, 332, 334, $337,339,343$

Streptococcus faecalis var. liquefaciens (Stennberg) Mattick, 265, 300, 301, 308, $309,310,324,325,341,344$

Stysanus sp., 301

Synnematium jonesii Speare, 266

$\mathbf{T}$

Thelohania sp., 281, 284, 338

Thelohania fibrata (Lutz \& Splendor) Debaisieux \& Gastaldi, 284

Thelohania pinguis Hesse, 281

Thielavia sp., 344

Triboliocystis garnhami Dissanaike, 276

Trichoderma sp., 301

Trichoderma koningi Oudemans, 272

Tritirachium sp., 278

$\mathbf{v}$

Verticillium sp., 294, 301, 302, 307, 308, 344

Verticillium terrestre (Link) Lindau, 264 